UNIVERSIDADE DE SÃO PAULO

INSTITUTO DE FÍSICA

\title{
Uma estratégia Euclidiana para o estudo do efeito Unruh
}

\section{Pedro Tavares Paes Lopes}

Orientador: Prof. Dr. João Carlos Alves Barata

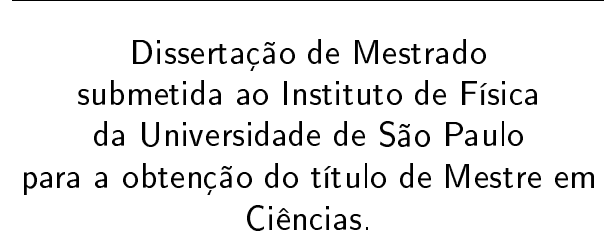

Ciências.

\section{Banca Examinadora:}

Prof. Dr. João Carlos Alves Barata (IF-USP)

Prof. Dr. Luiz Renato Gonçalves Fontes (IME-USP)

Prof. Dr. George Emanuel Avraam Matsas (IFT-UNESP)

São Paulo

2007 


\section{Resumo}

Neste trabalho nós propomos uma estratégia Euclidiana para entender o efeito Unruh. Com este objetivo, nós inicialmente o estudamos para campos livres escalares sem massa, numa forma que é normalmente apresentada aos físicos e que é mais próxima ao trabalho original de Unruh [32].

Logo em seguida, deduzimos o efeito de um ponto de vista algébrico. Com este objetivo, estudamos as propriedades e as definições de estados KMS para compreender como um estado de equilíbrio é descrito na abordagem algébrica. Apresentamos os axiomas de Wightman para campos escalares assim como os de Osterwalder-Schrader. Usamos, então, o Teorema de Bisognano-Wichmann para estes campos e concluímos, baseados no trabalho de Sewell [27], que um observador uniformemente acelerado vê o estado de vácuo dos observadores inerciais como um estado KMS, e portanto, como um estado de equilíbrio. Novamente, concluímos a existência do efeito Unruh.

Finalmente estudamos algumas relações entre probabilidade e análise funcional. Este estudo é fundamental para o entendimento do trabalho de Klein e Landau [15] e de Gérard e Jäkel [7]. Estes trabalhos afirmam que existe uma relação biunívoca entre certos estados KMS e certos processos estocásticos (Klein e Landau) e uma relação entre certos processos estocásticos e espaços de trajetórias generalizados (Gérard e Jäkel). Usando estes trabalhos e as funções de Schwinger para campos escalares, deduzimos o efeito Unruh de uma nova maneira.

Acreditamos que este trabalho mostra um ponto de vista interessante do efeito Unruh e ilustra o uso do formalismo Euclidiano em teorias quânticas dos campos. Mesmo que algumas demonstrações para uma prova completa do efeito, usando técnicas Euclidianas, não são obtidas, devido às dificuldades técnicas encontradas, acreditamos que o material apresentado neste trabalho fornece, no mínimo, uma boa estratégia para a compreensão completa deste fenômeno físico. Além disto, as técnicas que são mostradas podem ser usadas em diversos problemas, como a construção de campos interagentes a uma temperatura finita, que permanecem atuais e promissores. 


\section{Abstract}

This paper proposes a Euclidean strategy to understand the Unruh effect. On that ground we first study it for free massless scalar fields the way it is usually presented to physicists, which is closer to Unruh's original work [32].

Then we infer the effect from an algebraic perspective. We study the proprieties and definitions of KMS states in order to understand the description of an equilibrium state in the algebraic approach. We present the Wightman's as well as Osterwalder-Schrader's axioms for scalar fields. Then we use the Bisognano-Wichmann theorem for these fields and conclude, based on Sewell work [27], that a uniformly accelerated observer will observe the vacuum state of inertial observers as a KMS state and thus as an equilibrium state. Once again we infer the existence of the Unruh effect.

Finally we study some relations between probability and functional analysis. This study is crucial for understanding the work of Klein and Landau [15] as well as of Gérard and Jäkel [7]. They state there is a biunivocal relation between certain KMS states and certain stochastic processes (Klein and Landau) and a relation between certain stochastic processes and generalized path spaces (Gérard and Jäkel). Using these works and Schwinger functions for scalar fields, we deduce the Unruh effect in a new way.

We believe this work shows an interesting aspect of the Unruh effect and represents the use of Euclidean formalism in quantum field theory. Although some demonstrations for a complete proof of the Unruh effect using Euclidean techniques were not obtained due to technical difficulties we faced, we believe the material presented in this paper provides at least a good strategy for the complete understanding of this physical phenomenon. Furthermore the techniques shown, which remain current and promising, can be used in different problems, such as the construction of interacting fields at a finite temperature. 


\section{Agradecimentos}

Gostaria aqui de expressar meus agradecimentos a todos que tornaram a realização desta dissertação possível.

Aos professores e funcionários do Instituto de Física da USP que permitiram a minha formação, a qual sempre me influenciará não importe qual atividade profissional que eu ainda vá seguir.

Ao meu orientador, João Carlos Alves Barata, pelo grande estímulo e por me ajudar a atravessar os caminhos da física-matemática.

A todos meus amigos, que dentro e fora da universidade sempre me apoiaram na dissertação e em tantas outras coisas da vida.

E, por fim, a minha família, sem a qual nada seria possível.

Ah, e claro, agradeço ao Conselho Nacional de Desenvolvimento Científico e Tecnológico (CNPq) e a Fundação de Amparo a Pesquisa do Estado de São Paulo (FAPESP) pelo apoio financeiro e por me financiarem tantas pizzas nas sextas-feiras! 


\section{Sumário}

1 Introdução $\quad 5$

2 O efeito Unruh: ponto de vista informal $\quad 8$

2.1 Efeito Unruh e referenciais acelerados . . . . . . . . . . . . . . . 8

2.2 Efeito Unruh: ponto de vista informal . . . . . . . . . . . . . . . . . 12

2.2.1 A quantização canônica do campo escalar livre no espaço de Minkowski . 12

2.2.2 A quantização canônica do campo escalar em espaços globalmente hiperbólicos. . . . . . . . . . . . . . . . . . . . . . . . 14

2.2.2.1 A quantização canônica em espaços globalmente hiperbólicos:

O trabalho de Dimock . . . . . . . . . . . . . . 18

2.2.3 O vácuo dos observadores inerciais visto por observadores uniformemente acelerados ........................ 20

3 O efeito Unruh: ponto de vista algébrico $\quad 26$

3.1 Propriedades fundamentais das álgebras de operadores . . . . . . . . . . . . 26

3.2 Estados KMS . . . . . . . . . . . . . . . . . . . . . . . . . . . . . . . 31

3.3 Axiomas de Wightman . . . . . . . . . . . . . . . . . . . . 39

3.4 O efeito Unruh de um ponto de visto algébrico (o trabalho de Sewell) . . . . . 45

4 O efeito Unruh: ponto de vista estocástico $\quad 48$

4.1 Relações entre probabilidade e análise funcional . . . . . . . . . . . . . 48

4.2 O trabalho de Klein e Landau . . . . . . . . . . . . . . . . . . . . . . 65

4.3 O efeito Unruh do ponto de vista estocástico . . . . . . . . . . . . . . . . 67

4.3.1 Espaço de trajetória generalizado . . . . . . . . . . . . . . 68

4.3.2 O Teorema de reconstrução . . . . . . . . . . . . . . . . . . 74

$\begin{array}{llr}5 & \text { Conclusão } & 79\end{array}$

$\begin{array}{ll}\text { Referências Bibliográficas } & 82\end{array}$ 


\section{Capítulo 1}

\section{Introdução}

Dentre as diversas formulações da teoria quântica dos campos, as chamadas teoria axiomática e teoria quântica local ou algébrica têm a vantagem de permitir um estudo de propriedades gerais, independente de modelos e aproximações. As aplicações dos métodos algébricos utilizadas nestas duas abordagens permitem criar uma estrutura rigorosa para a formulação tanto da teoria quântica dos campos quanto da mecânica estatística quântica.

Neste trabalho, usaremos a estrutura algébrica para o estudo do efeito Unruh. O efeito Unruh se insere no contexto da teoria quântica de campos em espaços curvos. Historicamente, de acordo com Robert Wald [34], o estudo da teoria de campos em espaços curvos iniciou-se no final dos anos 60. O estímulo inicial destes trabalhos foi o estudo da criação de partículas produzidas pela expansão do universo no período próximo ao "Big Bang". A estrutura da teoria apareceu para analisar estes fenômenos com o trabalho de Parker [25] entre outros. Logo após, um grande impulso foi recebido na década de 70 graças a descoberta, feita pelo físico Stephen Hawking [23] em 1975, de que os buracos negros irradiam tais como corpos negros devido a criação de partículas. Este efeito passou a ser chamado de efeito Hawking. Após essa descoberta, numa tentativa de ganhar uma intuição maior sobre ela e compreendê-la melhor, foi descoberto o efeito Unruh [32] em 1976, que será estudado nesta dissertação. Em termos não tão precisos, este efeito afirma que um observador uniformemente acelerado sente-se imerso num banho térmico, quando o campo quântico está no estado de vácuo em relação aos referenciais inerciais. Esses assuntos geraram, na década de 70, uma grande quantidade de estudos.

A formulação rigorosa da teoria de campos em espaços curvos utiliza-se dos métodos algébricos citados anteriormente, em especial da abordagem algébrica iniciada por Haag e Kastler [22]. No caso do efeito Unruh uma explicação mais rigorosa já existia antes mesmo de Unruh descobri-lo. Em [9, 10], Bisognano e Wichmann já mostravam que, para campos restritos à cunha de Rindler, $\left\{(c t, x, y, z) \in \mathbb{R}^{4} ; x>c|t|\right\}$ (c é a velocidade da luz), o estado de vácuo dos observadores inerciais é um estado KMS em relação aos observadores uniformemente acelerados (que correspondem ao boost de Lorentz). Apesar deste artigo já conter o resultado matemático anterior, os autores não notaram (ou ao menos não deixaram explícito) o efeito físico que este resultado implicava. Foi só com o trabalho de Sewell [27] que o trabalho de Bisognano e Wich- 
mann $[9,10]$ foi usado para estabelecer uma formulação mais precisa, matematicamente, do efeito Unruh.

A abordagem algébrica devido a Haag e Kastler (entre outros) surgiu ao longo dos anos 60 na mesma época em que surgia os chamados axiomas de Wightman, ou seja, a formulação axiomática da teoria de campos. Como conseqüência destes trabalhos, surgiram problemas como o de construir campos quânticos não-triviais que satisfizessem os axiomas de Wightman e, junto com estas questões, a formulação Euclidiana da teoria de campos. Em termos bem informais, a formulação da teoria de campos na forma Euclidiana corresponde à troca do tempo real por um imaginário $t \rightarrow i t$, substituindo-se, desta forma, o espaço de Minkowski pelo Euclidiano.

A formulação Euclidiana também mostrou que existem relações entre a teoria quântica de campos e a mecânica estatística clássica, ao tornar evidente que o problema da construção de campos interagentes é essencialmente reduzido a um problema de mecânica estatística (vide por exemplo [28]). Dentre os trabalhos desta natureza se destacam vários autores. Num destes artigos, escrito por Abel Klein e Lawrence Landau [15], se estabelece uma relação biunívoca entre certos estados KMS e certos processos estocásticos. Este trabalho tem o mesmo espírito de relacionar métodos de teorias quânticas (álgebras não-comutativas, tais como álgebras de von Neumann e estados KMS) com métodos mais usuais em mecânica estatística (álgebras comutativas, tais como funções de um processo estocástico). Ele permite assim estudar perturbações de certos estados KMS. O mais curioso, para nós pelo menos, é que este trabalho permite a construção de estados de temperatura finita e não-nula de campos quânticos tal qual foi mostrado por Gérard e Jäkel [7].

O interessante nestes dois artigos é que, para alguns resultados do trabalho [15], basta que tenhamos um espaço de trajetória generalizado tal como mostrado em [7]. Este último trabalho estabelece uma relação biunívoca entre espaços de trajetória generalizados e os mesmos tipos de estados KMS apresentados no trabalho de [15]. O espaço de trajetória generalizado a que nos referimos é análogo ao espaço de trajetória encontrado em textos como o do Barry Simon [28].

Acredita-se que se possa usar as idéias contidas nestes dois artigos a fim de demonstrar o efeito Unruh de forma alternativa.

A idéia é mostrar que o campo livre Euclidiano pode ser descrito por meio de um espaço de trajetória generalizado se tomarmos como tempo Euclidiano a rotação $\tau$ ao longo dos eixos $x$ e it. Esta rotação, como se sabe, corresponde ao boost de Lorentz "Euclidianizado", ou seja, ao invés de uma "rotação" de $c t \rightarrow c t \cosh \tau+x \sinh \tau$ e $x \rightarrow x \cosh \tau+c t \sinh \tau$ no espaço de Minkowski, temos uma "rotação" por $i \tau$ no espaço Euclidiano: $i c t \rightarrow i c t \cos (\tau)+i x \sin (\tau)$ e $x \rightarrow x \cos (\tau)-c t \sin (\tau)$. Logo o tempo Euclidiano, tomado como a rotação de um ângulo $\tau$, corresponde ao tempo Euclidiano de um observador uniformemente acelerado (já que para este, o tempo real é proporcional a $\tau$ do boost de Lorentz). Se estabelecermos esta conexão podemos usar o Teorema de [15] e associar o espaço de trajetória generalizado a um estado KMS, cuja 
evolução temporal Euclidiana é dada por $\tau$ e, portanto, cuja evolução temporal real é dada pelo tempo próprio de observadores uniformemente acelerados. Concluiremos assim que ao campo quântico, visto por um observador uniformemente acelerado, corresponde a um estado KMS. Dessa forma obtemos uma nova explicação do efeito Unruh.

No trabalho apresentado nesta tese não se chegará a fazer a demonstração alternativa, apenas esboçaremos qual é a estratégia para tanto. O que será apresentado é, inicialmente, o efeito Unruh do ponto de vista informal (ou seja, do ponto de vista da teoria de campos tal como ela é apresentada usualmente em cursos de física) e que de certa forma se assemelha mais com o trabalho original de Unruh [32]. Isto será feito no capítulo 2, juntamente com uma descrição (bem pouco rigorosa) da formulação da teoria de campos em espaços curvos e uma prova da relação entre os boosts de Lorentz e observadores uniformemente acelerados. Isto tudo será feito para o caso de bósons.

No capítulo 3, mostraremos o efeito Unruh do ponto de vista de Sewell [27]. Este ponto de vista é matematicamente mais preciso e será mostrado junto com uma discussão dos axiomas de Wightman e sua Euclidianização (axiomas de Osterwalder-Schrader). Também apresentaremos uma discussão dos estados KMS que serão essenciais para este ponto de vista mais rigoroso. Nestas discussões pretendemos apresentar algumas demonstrações de fatos básicos conhecidos, porém não encontrados facilmente na literatura.

Por fim, no capítulo 4 esboçaremos a nova estratégia para explicar o efeito Unruh. Assim, começaremos explicando algumas relações entre análise funcional e teoria de probabilidade que será essencial para compreendermos os trabalhos de [15, 7]. Novamente os resultados apresentados nesta seção são certamente conhecidos, porém muitas das demonstrações não foram encontradas na literatura. Após fazer estas explicações, descreveremos o trabalho de Klein e Landau [15] e o trabalho de Christian Gérard e Christian Jäkel [7]. Mostraremos, enfim, como os dois trabalhos se relacionam e qual seria um possível procedimento para fazer uma demonstração alternativa do efeito Unruh para partículas bosônicas.

Durante o trabalho usaremos, em geral, unidades de velocidade da luz e constante de Planck como sendo $c=1$ e $\hbar=1$. Esta convenção não será respeitada apenas em algumas partes, quando gostaríamos de deixar estas constantes explícitas. 


\section{Capítulo 2}

\section{O efeito Unruh: ponto de vista informal}

Neste capítulo vamos fazer um breve apanhado, sem muita preocupação com o rigor matemático, de como formular a teoria quântica dos campos em espaços curvos. Basearemos o texto principalmente no livro de Birrel e Davies [16]. Vamos nos concentrar particularmente nos campos livres e bosônicos, ou seja, que satisfazem a equação de Klein-Gordon. Inicialmente faremos um breve apanhado do significado de isometrias em espaços-tempos, a fim de relacioná-lo com a interpretação de partícula. Relacionaremos um tipo de isometria do espaço de Minkowski, a dos boosts de Lorentz, com os observadores uniformemente acelerados.

A seguir, lembraremos a quantização canônica em espaços de Minkowski. Então, adaptaremos esta quantização aos espaços globalmente hiperbólicos e mostraremos como se relacionam os vácuos obtidos devido a quantizações diferentes em um mesmo espaço-tempo, obtendo os coeficientes de Bogolubov. Por fim, usaremos estes coeficientes para relacionar o vácuo dos observadores uniformemente acelerados com os observadores em um referencial inercial, obtendo o efeito Unruh, baseando-se principalmente em [20] para esta parte.

\subsection{Efeito Unruh e referenciais acelerados}

Estaremos interessados em estudar nesta seção a teoria de campos em espaços curvos. Esta teoria, embora quântica, trata o espaço-tempo classicamente. Assim descreveremos o espaçotempo $\left(M, g_{\mu \nu}\right)$ como sendo $M$ uma variedade diferenciável $C^{\infty}$ quadridimensional e $g_{\mu \nu}$ uma métrica Lorentziana com assinatura $(+---)$. É neste espaço-tempo onde se constroem os campos de matéria quânticos.

Ao fazermos a quantização de campos nestes espaços-tempos curvos arbitrários, fatores novos aparecem que não eram aparentes na teoria de campos no espaço de Minkowski. Percebemos, por exemplo, que nem sempre é possível obter uma interpretação precisa do conceito de partícula. Um pré-requisito para que possamos atribuir uma definição de partícula é a existência de um campo de Killing global tipo tempo. A idéia é, ao quantizar um campo, considerar soluções de freqüência positiva em relação a um campo de Killing, que fará o papel de trajetória dos observadores sobre a qual o conceito de partícula se aplica. 
Passemos agora a explicar o que é este campo de Killing global (baseado no livro do Wald [33]). Dado um espaço-tempo $\left(M, g_{\mu \nu}\right)$, chamamos de grupo uniparamétrico um conjunto de funções $\left\{\phi_{t}\right\}_{t \in \mathbb{R}}$ tais que $\phi_{t}: \mathbb{R} \times M \rightarrow M$ são funções $C^{\infty}$ cuja sua restrição a cada valor $t$ em $\mathbb{R}$ é um difeomorfismo de M em M. Estas funções devem satisfazer a propriedade $\phi_{t} \circ \phi_{s}=\phi_{t+s}$, o que implica que $\phi_{0}=i d$, em que $i d$ é a função identidade.

Dizemos que este grupo uniparamétrico é uma isometria se $\phi_{t}^{*} g_{\mu \nu}=g_{\mu \nu}$. Em termos de componentes isto pode ser visto da seguinte forma. Vamos considerar um ponto $p \in M,\left\{x^{\mu}\right\}$ um sistemas de coordenadas de p e $\left\{y^{\mu}\right\}$ um sistema de coordenadas de $\phi_{t}(p)$, os quais satisfazem $y^{\mu}=\phi_{t}\left(x^{\mu}\right)$. Então temos uma isometria se, e somente se,

$$
g_{\mu \nu}(y) \frac{\partial y^{\mu}}{\partial x^{\sigma}} \frac{\partial y^{\nu}}{\partial x^{\rho}}=g_{\sigma \rho}(x)
$$

Associado a cada grupo uniparamétrico existe um campo de vetores $\xi$, chamado de gerador, tal que $\xi$ em um ponto $p \in M$ é a tangente da curva $\phi_{t}(p): \mathbb{R} \rightarrow M$ (da mesma forma associado a cada campo de vetores infinitamente diferenciáveis, temos um grupo uniparamétrico). Se o grupo uniparamétrico é de isometrias, chamamos o campo de vetores gerador de campo de Killing.

A partir destas definições podemos definir um espaço-tempo que possui um campo de Killing global de tipo tempo como sendo um espaço-tempo que possui um grupo uniparamétrico de isometrias cujo campo de vetores gerador, campo de Killing, é um campo de vetores tipo tempo.

Como aplicação deste conceito, podemos ter no espaço-tempo de Minkowski tanto um grupo uniparamétrico de isometrias dado por translações temporais como dado pelos boosts de Lorentz. Vamos, então, ver porque estes grupos uniparamétricos correspondem a isometrias.

O grupo uniparamétrico de translações temporais é dado por funções da forma $\phi_{\tau}(t, x, y, z)=$ $(t+\tau, x, y, z)$. Desta forma vemos que, na notação acima, para um ponto $p=(t, x, y, z)$ as coordenadas $y^{\mu}$ de $\phi_{\tau}(p)$ serão dadas por $(t+\tau, x, y, z)$ e assim $\frac{\partial y^{\mu}}{\partial x^{\nu}}=\delta_{\nu}^{\mu}$ e, portanto, evidentemente a equação 2.1 é satisfeita. Também podemos notar que $\frac{d}{d \tau} \phi_{\tau}(p)=(1,0,0,0)$. Logo o vetor gerador $\xi$ das translações temporais é de tipo tempo. Concluímos assim que as translações temporais correspondem a um grupo uniparamétrico de isometrias com vetor de Killing tipo tempo. Assim, o espaço-tempo de Minkowski possui um campo global de Killing tipo tempo dado pelas translações temporais.

Quanto ao grupo uniparamétrico de isometrias de boosts de Lorentz, vamos nos restringir ao caso em que os boosts ocorrem na direção $x$. O grupo uniparamétrico será dado então por $\phi_{\tau}(t, x, y, z)=(t \cosh \tau+x \sinh \tau, x \cosh \tau+t \sinh \tau, y, z)$. Assim obtemos que:

$$
\frac{\partial y^{\mu}}{\partial x^{\nu}}=\left(\begin{array}{cccc}
\cosh \tau & \sinh \tau & 0 & 0 \\
\sinh \tau & \cosh \tau & 0 & 0 \\
0 & 0 & 1 & 0 \\
0 & 0 & 0 & 1
\end{array}\right)=\Lambda(\tau)_{\nu}^{\mu}
$$


Como $\Lambda(\tau)_{\nu}^{\mu}$ é uma transformação e Lorentz correspondente aos boosts, a equação 2.1 pode ser escrita da forma:

$$
g_{\mu \nu}(y) \frac{\partial y^{\mu}}{\partial x^{\sigma}} \frac{\partial y^{\nu}}{\partial x^{\rho}}=g_{\mu \nu}(y) \Lambda(\tau)_{\sigma}^{\mu} \Lambda(\tau)_{\rho}^{\mu}=g_{\sigma \rho}(x) .
$$

Logo concluímos que o boost na direção $x$ é um grupo uniparamétrico de isometrias. Para obter o campo gerador basta notar que $\frac{d}{d \tau} \phi_{\tau}(t, x, y, z)=(t \sinh \tau+x \cosh \tau, x \sinh \tau+$ $t \cosh \tau, 0,0)$. Notamos que o quadrado (na métrica de Lorentz) deste vetor é $x^{2}-t^{2}$. Portanto no espaço de Rindler, ou cunha de Rindler, $\mathcal{R}=\left\{(t, x, y, z) \in \mathbb{R}^{4} ;|t|<x\right\}$, o campo de Killing é tipo tempo. Assim podemos dizer que o espaço de Rindler é um espaço-tempo que possui um campo global de Killing tipo tempo dado pelos boosts de Lorentz.

Se fizermos a quantização do campo, utilizando como tempo as translações temporais, obtemos a interpretação de partículas convencional. Se fizermos a quantização do campo restrito à cunha de Rindler utilizando como tempo os boosts de Lorentz na direção $x$, obtém-se a interpretação das chamadas partículas de Rindler. O "tempo" dado como sendo os boosts de Lorentz têm a interpretação de ser o tempo tal qual visto por um observador uniformemente acelerado (o tempo próprio). Uma explicação de como fazer a quantização nestes casos, sem muita preocupação com o rigor matemático, pode ser vista na seção 1.2.

Seguindo este raciocínio, pode-se dizer que o efeito Unruh afirma, de forma bastante curta, que um observador uniformemente acelerado, com aceleração própria $\omega$ e estando no vácuo de observadores inerciais, detecta um banho térmico de partículas elementares de Rindler à temperatura $T_{U}=\frac{\hbar \omega}{2 \pi k c}$.

Para entender a formalização deste resultado vamos inicialmente entender um fato simples e básico na discussão acima: Os boosts de Lorentz na direção $x, t \rightarrow t \cosh \tau+x \sinh \tau$ e $x \rightarrow x \cosh \tau+t \sinh \tau$, restritos à cunha de Rindler correspondem a translações temporais $\tau \rightarrow \tau+$ cte (tempo próprio) para observadores uniformemente acelerados, particularmente soluções em que $x^{\mu}(0)=\left(0, \frac{c^{2}}{\omega}, 0,0\right)$ e $\frac{d x^{\mu}}{d s}(0)=(1,0,0,0)$ (veja notação abaixo) em que $\omega$ é a aceleração da partícula nos referenciais instantaneamente parados. Seguiremos aqui os passos de Landau e Lifschitz [11].

Vamos, agora, usar unidades com a velocidade da luz $c$ ao invés de 1, a fim de entendê-las melhor.

Seja $(c t, x, y, z)$ uma parametrização do espaço de Minkowski num referencial inercial. Seja $\tau=\frac{s}{c}$ o tempo próprio do observador que se relaciona com $\mathrm{t}$ por $\frac{d s}{d t}=c \sqrt{1-\frac{v^{2}}{c^{2}}}$. Defino as quadrivelocidades e quadriaceleração por $v^{\mu}=\frac{d x^{\mu}}{d s}$ e $\omega^{\mu}=\frac{d^{2} x^{\mu}}{d s^{2}}$. Assim $v^{\mu}=\frac{d x^{\mu}}{d s}=$ $\left(\frac{1}{\sqrt{1-\frac{v^{2}}{c^{2}}}}, \frac{\mathbf{v}}{c \sqrt{1-\frac{v^{2}}{c^{2}}}}\right)$. Vemos assim que $v^{\mu} v_{\mu}=g_{\mu \nu} \frac{d x^{\mu}}{d s} \frac{d x^{\nu}}{d s}=1$. Derivando esta relação em $s$ obtemos $v^{\mu} \omega_{\mu}=g_{\mu \nu} \frac{d x^{\mu}}{d s} \frac{d^{2} x^{\nu}}{d s^{2}}=0$.

Como estamos interessados numa aceleração uniforme ao longo do eixo $x$, temos que em relação ao tempo próprio $x^{\mu}(\tau)=(c t(\tau), x(\tau), y, z)$, em que $y$ e $z$ são constantes. Como a aceleração é uniforme, gostaríamos que ao longo de qualquer referencial inercial instantaneamente 
parado, ou seja, de velocidade nula em relação ao observador, tivéssemos $\omega^{\mu}=\left(0, \frac{\omega}{c^{2}}, 0,0\right)$ em que $\omega$ é a aceleração e é constante (uniforme). Logo, nestes referenciais (e em todos os referenciais inerciais por conseqüência) temos que $\omega^{\mu} \omega_{\mu}=-\frac{\omega^{2}}{c^{4}}$, o que implica:

$$
\omega^{\mu} \omega_{\mu}=\frac{1}{c^{2}-v^{2}}\left[\left(\frac{d}{d t} \frac{1}{\sqrt{1-\frac{v^{2}}{c^{2}}}}\right)^{2}-\left(\frac{d}{d t} \frac{v}{c \sqrt{1-\frac{v^{2}}{c^{2}}}}\right)^{2}\right]=-\frac{\omega^{2}}{c^{2}}
$$

Usando $v^{\mu} \omega_{\mu}=0$, obtemos a relação:

$$
\frac{d}{d t} \frac{1}{\sqrt{1-\frac{v^{2}}{c^{2}}}}=\frac{v}{c} \frac{d}{d t} \frac{v}{c \sqrt{1-\frac{v^{2}}{c^{2}}}} .
$$

Substituindo a relação acima em 2.2, obtemos:

$$
\frac{d}{d t} \frac{v}{c \sqrt{1-\frac{v^{2}}{c^{2}}}}=\frac{\omega}{c}
$$

Usando a condição inicial $v(0)=0$, chegamos à:

$$
\frac{v}{\sqrt{1-\frac{v^{2}}{c^{2}}}}=\omega t \therefore v=\frac{\omega t}{\sqrt{1+\frac{\omega^{2} t^{2}}{c^{2}}}} .
$$

Usando novamente as condições iniciais em que $x(0)=\frac{c^{2}}{\omega}$ obtemos:

$$
x(t)=\frac{c^{2}}{\omega}+\int_{0}^{t} \frac{\omega y}{\sqrt{1+\frac{\omega^{2}}{c^{2}} y^{2}}} d y=\frac{c^{2}}{\omega} \sqrt{1+\frac{\omega^{2}}{c^{2}} t^{2}} .
$$

A função $t(\tau)$, em que $\tau$ é o tempo próprio pode ser calculada por

$$
\tau=\int_{0}^{t} \sqrt{1-\frac{v\left(t^{\prime}\right)^{2}}{c^{2}}} d t^{\prime}=\int_{0}^{t} \frac{1}{\sqrt{1+\frac{\omega^{2} y^{2}}{c^{2}}}} d y=\frac{c}{\omega} \operatorname{arcsinh}\left(\frac{\omega}{c} t\right) .
$$

Isolando, obtemos o resultado principal $x^{\mu}(\tau)=\left(\frac{c^{2}}{\omega} \sinh \left(\frac{\omega}{c} \tau\right), \frac{c^{2}}{\omega} \cosh \left(\frac{\omega \tau}{c}\right), y, z\right)$.

Assim se parametrizarmos o espaço de Rindler $\mathcal{R}$ por:

$$
\begin{gathered}
c t=\xi \sinh T \\
x=\xi \cosh T \\
y=y \\
z=z
\end{gathered} .
$$

Concluímos, comparando as expressões, que $\xi=\frac{c^{2}}{\omega}$ e $T=\frac{\omega \tau}{c}$. Logo as trajetórias com $\xi$ constante correspondem a trajetórias uniformemente aceleradas com aceleração $\omega=\frac{c^{2}}{\xi}$ e tempo próprio dado por $\tau=\frac{c}{\omega} T$.

Se parametrizarmos o espaço de Rindler $\mathcal{R}$ por: 


$$
c t=\frac{1}{a} e^{a \xi} \sinh (a \eta) \quad x=\frac{1}{a} e^{a \xi} \cosh (a \eta) .
$$

Em que $a>0$ é uma constante, obtemos $\frac{1}{a} e^{a \xi}=\frac{c^{2}}{\omega}$, assim $\omega=c^{2} a e^{-a \xi}$. Também obtemos $a \eta=\frac{\omega}{c} \tau$, assim $\tau=\frac{\eta}{c} e^{a \xi}$. Estas duas parametrizações do espaço de Rindler foram expostas aqui porque serão usadas a seguir, na explicação do efeito Unruh.

\subsection{Efeito Unruh: ponto de vista informal}

Nesta seção faremos uma exemplificação do efeito Unruh para campos escalares de um ponto de vista informal. Para tanto, seguiremos de perto a referência [16]. A proposta será apresentar um resumo da quantização de campos escalares para espaços de Minkowski e, então, mostrar como se quantiza em espaços-tempos globalmente hiperbólicos. Ilustraremos assim o uso do grupo uniparamétrico de isometrias com campo de vetores de Killing globalmente tipo tempo. Mostraremos com isto uma segunda forma de quantizar o campo em espaços de Minkowski para então comparar o vácuo das duas formas de quantização.

\subsubsection{A quantização canônica do campo escalar livre no espaço de Minkowski}

Inicialmente vamos começar com um breve resumo da quantização canônica em espaços de Minkowski. A idéia é quantizar um campo escalar livre real, que pode ou não ter massa, que satisfaça a equação de Klein-Gordon (para $m \geq 0$ ), ou seja,

$$
\left(\square+m^{2}\right) \phi=0
$$

Lembrando que esta equação pode ser obtida de um ponto de vista variacional fazendo a variação do funcional ação $S$ e igualando esta variação a $0, \delta S=0$. O funcional ação no caso é dado por:

$$
S=\int \mathcal{L}(x) d^{n} x=\frac{1}{2} \int\left(\eta^{\alpha \beta} \frac{\partial \phi}{\partial^{\alpha} x} \frac{\partial \phi}{\partial^{\beta} x}-m^{2} \phi^{2}\right) d^{n} x .
$$

Um conjunto de soluções para a equação de Klein-Gordon pode ser dada por:

$$
u_{\vec{k}}(t, \vec{x})=\frac{1}{\sqrt{2 \omega(2 \pi)^{n-1}}} e^{i \vec{k} \cdot \vec{x}-i \omega t},
$$

em que $\omega=\sqrt{k^{2}+m^{2}}$ e $k=|\vec{k}|=\sqrt{k_{1}^{2}+\ldots+k_{n-1}^{2}}$ para $k_{i} \in \mathbb{R}$.

Estas soluções são autovetores do operador de derivação temporal, isto é,

$$
\frac{\partial}{\partial t} u_{\vec{k}}(t, \vec{x})=-i \omega u_{\vec{k}}(t, \vec{x})
$$


Se definirmos uma forma sesquilinear por:

$$
\left(\phi_{1}, \phi_{2}\right)=-i \int\left\{\phi_{1}(x)\left[\partial_{t} \phi_{2}^{*}(x)\right]-\left[\partial_{t} \phi_{1}(x)\right] \phi_{2}^{*}(x)\right\} d x^{n-1} \equiv-i \int \phi_{1}(x) \overleftrightarrow{\partial_{t}} \phi_{2}^{*}(x) d^{n-1} x
$$

Concluiremos que $\left(u_{\vec{k}}, u_{\vec{k}^{\prime}}\right)=\delta^{n-1}\left(\vec{k}-\vec{k}^{\prime}\right)$. Se preferirmos fazer a quantização numa caixa ao invés do espaço de Minkowski inteiro, ou seja, num conjunto $\mathbb{R} \times\left[-\frac{L}{2}, \frac{L}{2}\right]^{n-1}$, poderíamos escolher as funções como sendo dadas por:

$$
u_{\vec{k}}(\vec{x}, t)=\frac{1}{\sqrt{2 L^{n-1} \omega}} e^{i \vec{k} \cdot \vec{x}-i \omega t} .
$$

Sendo $k_{i}=\frac{2 \pi n}{L}, n \in \mathbb{N}$. Neste caso a normalização fica $\left(u_{\vec{k}}, u_{\vec{k}^{\prime}}\right)=\delta_{\vec{k} \vec{k}^{\prime}}$. Como esta é uma base completa podemos escrever o campo como:

$$
\phi(t, \vec{x})=\sum_{\vec{k}}\left[a_{\vec{k}} u_{\vec{k}}(t, \vec{x})+a_{\vec{k}}^{\dagger} u_{\vec{k}}^{*}(t, \vec{x})\right]
$$

A quantização ocorre no momento em que impomos que $\phi, \pi, a_{\vec{k}}, a_{\vec{k}}^{\dagger}$, em que $\pi(t, \vec{x})=$ $\frac{\partial \mathcal{L}}{\partial\left(\partial_{t} \phi\right)}=\partial_{t} \phi$, são operadores que satisfazem as relações de comutação para tempo iguais:

$$
\left[\phi(t, \vec{x}), \phi\left(t, \vec{x}^{\prime}\right)\right]=0\left[\pi(t, \vec{x}), \pi\left(t, \vec{x}^{\prime}\right)\right]=0\left[\phi(t, \vec{x}), \pi\left(t, \vec{x}^{\prime}\right)\right]=i \delta^{n-1}\left(\vec{x}-\vec{x}^{\prime}\right) .
$$

Estas relações equivalem a:

$$
\left[a_{\vec{k}}, a_{\vec{k}^{\prime}}\right]=0\left[a_{\vec{k}}^{\dagger}, a_{\vec{k}^{\prime}}^{\dagger}\right]=0\left[a_{\vec{k}}, a_{\vec{k}^{\prime}}^{\dagger}\right]=\delta_{\vec{k} \vec{k}^{\prime}}
$$

Estes operadores são pensados como atuando em um espaço de Fock simétrico construído a partir de um espaço de Hilbert $\mathcal{H}$. Tomaremos o espaço $\mathcal{H}$ como sendo o completamento do espaço de vetorial das combinações lineares das funções $u_{\vec{k}}$ no produto interno (., .). As funções $u_{\vec{k}}$ são neste caso uma base deste espaço como mostra a proposição abaixo:

PROPOSIÇÃo 2.2.1.1: Seja um espaço vetorial $\mathcal{E}$ com produto interno e formado por combinações lineares de elementos $\left\{f_{j}\right\}_{j \in \mathbb{N}}$, em que $f_{j}$ são elementos ortonormais. Então $\left\{f_{j}\right\}_{j \in \mathbb{N}}$ é uma base do espaço de Hilbert construído a partir do completamento canônico de $\mathcal{E}, \overline{\mathcal{E}}$.

Prova: No completamento canônico temos que $\mathcal{E}$ é denso em $\overline{\mathcal{E}}$ e $\left\{f_{j}\right\}_{j \in N}$ continua sendo ortonormal $\left(\mathcal{E}\right.$ e $\left\{f_{j}\right\}_{j \in N}$ visto como os elementos correspondentes em $\overline{\mathcal{E}}$, lembrando que no completamento canônico existe uma isometria entre $\mathcal{E}$ e $\overline{\mathcal{E}}$ ). Logo se existe um vetor $g \neq 0$ que é ortogonal a qualquer $f_{j}$ concluímos que $g$ é ortogonal a $\mathcal{E}$ e, portanto, $\mathcal{E}$ não é denso. Obtemos, então, uma contradição. Assim $\left\{f_{j}\right\}_{j \in N}$ é um conjunto maximal de elementos ortogonais e, desta forma, uma base.

O espaço de Fock, construído a partir de um espaço de Hilbert $\mathcal{H}$, será denotado por $\mathcal{F}(\mathcal{H})$ 
e é definido por

$$
\mathcal{F}(\mathcal{H})=\mathbb{C} \oplus \bigoplus_{k=1}^{\infty} \mathcal{H}^{(n)}
$$

para $\mathcal{H}^{(n)}$ definido como $\mathcal{H}^{(n)}=\otimes_{k=1}^{n} \mathcal{H}$, ou seja, o produto tensorial de $n$ cópias de $\mathcal{H}$. Ao vetor $1 \oplus 0 \oplus(0 \otimes 0) \oplus \ldots$ damos o nome de vácuo e denotamo-lo, em geral, por $|0\rangle$. Em cada um dos espaços $\mathcal{H}^{(n)}$ pode-se construir o operador de simetrização por:

$$
S^{(n)}\left(f_{1} \otimes \ldots \otimes f_{n}\right)=\frac{1}{n !} \sum_{\pi} f_{\pi_{1}} \otimes \ldots \otimes f_{\pi_{n}},
$$

a soma sendo feita para todas as permutações, $\pi$, do conjunto $\{1, \ldots, n\}$. Este operador de simetrização pode ser expandido por linearidade e depois pelo Teorema BLT [6], já que é contínuo e densamente definido, a todo o espaço $\mathcal{H}$. Pode-se verificar que o operador $S^{(n)}$ assim definido é um operador de projeção ortogonal. Dizemos que o subespaço fechado dado pela imagem de $S^{(n)}$ é o espaço dos estados bosônicos da teoria.

O subespaço dos estados bosônicos do espaço de Fock será definido como o subespaço projetado por $S=\oplus_{n=0}^{\infty} S^{(n)}$. Este subespaço é chamado de espaço de Fock simétrico e é, evidentemente, também um espaço de Hilbert. Ele é o espaço dos estados de uma teoria bosônica tal como a descrita nesta seção.

Com estas definições, os operadores $a_{\vec{k}}$ e $a_{\vec{k}}^{\dagger}$ serão pensados agora como operadores de criação e aniquilação no espaço de Fock simétrico definidos como:

$$
a_{\vec{k}}=S a(\vec{k}) S \quad a_{\vec{k}}^{\dagger}=S a^{\dagger}(\vec{k}) S
$$

Os operadores $a(\vec{k})$ e $a^{\dagger}(\vec{k})$ são definidos em $\mathcal{H}^{(n)}$ como:

$$
\begin{gathered}
a(\vec{k}) f_{1} \otimes f_{2} \otimes \ldots \otimes f_{n}=n^{\frac{1}{2}}\left(u_{\vec{k}}, f_{1}\right) f_{2} \otimes \ldots \otimes f_{n} \\
a^{\dagger}(\vec{k}) f_{1} \otimes f_{2} \otimes \ldots \otimes f_{n}=(n+1)^{\frac{1}{2}} u_{\vec{k}} \otimes f_{1} \otimes \ldots \otimes f_{n}
\end{gathered}
$$

em que $f_{1} \otimes \ldots \otimes f_{n} \in \mathcal{H}^{(n)}$. A partir destas definições obtemos as relações de comutação canônicas dadas anteriormente.

\subsubsection{A quantização canônica do campo escalar em espaços global- mente hiperbólicos.}

Consideraremos agora a quantização em espaços-tempos mais gerais (conforme Birrel-Davies [16]). Vamos considerar o espaço-tempo como uma variedade $C^{\infty}, n$-dimensional em que está definida uma métrica $g_{\mu \nu}$ Lorentziana como já foi dito anteriormente. Além disto, vamos trabalhar apenas com espaços-tempos globalmente hiperbólicos. É conveniente, portanto, que 
gastemos algumas linhas definindo estes espaços (tal qual feito em [33]).

Num espaço-tempo dizemos que um ponto é um ponto final futuro de uma curva $\lambda: \mathbb{R} \rightarrow M$ se, para qualquer vizinhança $O_{p}$ de $p$, existe um $t_{0} \in \mathbb{R}$ tal que $\lambda(t) \in O_{p}$ para $t>t_{0}$. A curva é inextensível ao futuro se não tem nenhum ponto final no futuro. De forma completamente análogo se define uma curva inextensível ao passado.

A partir deste conceito, podemos definir o domínio de dependência de um conjunto fechado e acronal, $S$, (ou seja, tal que não existe uma curva tipo tempo ligando dois de seus pontos) da seguinte forma:

$D^{+}(S)=\{p \in M ;$ toda curva inextensível ao passado que passa por $\mathrm{p}$ intersecta $\mathrm{S}\}$

Novamente, de forma análoga defini-se $D^{-}(S)$. Dizemos que um conjunto fechado e acronal é uma superfície de Cauchy se $M=D^{+}(S) \cup D^{-}(S)$. Pode-se mostrar que uma superfície de Cauchy é uma subvariedade contínua e que todo subespaço que contém uma superfície de Cauchy contém infinitas outras, podendo ser folheado por elas. Desta forma, existe uma função $f: M \rightarrow \mathbb{R}$ tal que $f^{-1}(t)$ é superfície de Cauchy para qualquer que seja $t \in \mathbb{R}$. Um espaço-tempo que possui uma superfície de Cauchy é chamado espaço globalmente hiperbólico.

Para estes espaços-tempos consideraremos a ação do campo escalar livre dada por:

$$
S=\frac{1}{2} \int[-g(x)]^{\frac{1}{2}}\left\{g^{\mu \nu}(x) \phi(x),_{\mu} \phi(x),_{\nu}-\left[m^{2}+\xi R(x)\right] \phi^{2}(x)\right\} d^{n} x .
$$

A variação desta ação nos dá a equação:

$$
\left[\square_{x}+m^{2}+\xi R(x)\right] \phi(x)=0 .
$$

De forma completamente análoga ao espaço de Minkowski, definiremos um produto interno para as soluções da equação acima como:

$$
\left(\phi_{1}, \phi_{2}\right)=-i \int_{\Sigma} \phi_{1}(x) \overleftrightarrow{\partial_{\mu}} \phi_{2}^{*}(x)\left[-g_{\Sigma}(x)\right]^{\frac{1}{2}} d \Sigma^{\mu}
$$

Em que $d \Sigma^{\mu}=n^{\mu} d \Sigma$, sendo $n^{\mu}$ um vetor unitário dirigido ao futuro ortogonal à $\Sigma$ (superfície de Cauchy) e $d \Sigma$ é um elemento de volume da superfície de Cauchy. O produto interno acima independe da superfície de Cauchy, $\Sigma$, tomada, assim como a álgebra dos operadores dos observáveis também independerá, como será visto na seção 2.2.2.1.

Prova-se (de acordo com [16]) que, para estes espaços-tempos, existem bases completas de soluções da equação de onda $\left\{u_{i}\right\}$. Notemos, no entanto, que nem sempre estas funções são normalizadas e as somas abaixo muitas vezes deveriam ser substituídas por integrais. Um procedimento mais preciso para a quantização será mostrado numa seção posterior.

Estas soluções satisfazem as relações: 


$$
\left(u_{i}, u_{j}\right)=\delta_{i j}, \quad\left(u_{i}^{*}, u_{j}^{*}\right)=-\delta_{i j}, \quad\left(u_{i}^{*}, u_{j}\right)=0 .
$$

Novamente as funções de onda podem ser escritas como

$$
\phi(x)=\sum_{i}\left[a_{i} u_{i}(x)+a_{i}^{\dagger} u_{i}^{*}(x)\right]
$$

Agora, basta fazer a quantização canônica, ou seja, considerar o espaço de Hilbert $\mathcal{H}$ como sendo o completamento canônico do espaço vetorial das combinações lineares de $u_{j}$ com o produto interno definido anteriormente e definir o espaço de Fock simétrico construído a partir de $\mathcal{H}$, com seus operadores de criação e aniquilação $a_{i}$ e $a_{i}^{\dagger}$ satisfazendo:

$$
\left[a_{i}, a_{j}\right]=0, \quad\left[a_{i}^{\dagger}, a_{j}^{\dagger}\right]=0, \quad\left[a_{i}, a_{j}^{\dagger}\right]=\delta_{i j} .
$$

Se tivermos uma outra base de soluções $\left\{\bar{u}_{i}\right\}$ podemos escrever a função de onda como:

$$
\phi(x)=\sum_{j}\left[\bar{a}_{i} \bar{u}_{i}(x)+\bar{a}_{i}^{\dagger} \bar{u}_{i}^{*}(x)\right] .
$$

Podemos fazer a quantização canônica a partir daí. Note que, devido a completeza da base, podemos escrever $\bar{u}_{i}$ como:

$$
\bar{u}_{j}=\sum_{i}\left(\alpha_{j i} u_{i}+\beta_{j i} u_{i}^{*}\right)
$$

Levando em conta as relações de ortogonalidade de $u_{i}$ e que o produto interno, tal como foi construído, é linear na primeira entrada e anti-linear na segunda variável obtemos:

$$
\bar{u}_{j}=\sum_{i}\left[\left(\bar{u}_{j}, u_{i}\right) u_{i}-\left(\bar{u}_{j}, u_{i}^{*}\right) u_{i}^{*}\right]
$$

Logo concluímos que $\alpha_{i j}=\left(\bar{u}_{i}, u_{j}\right)$ e $\beta_{i j}=-\left(\bar{u}_{i}, u_{j}^{*}\right)$. Da mesma forma, mostra-se que

$$
u_{i}=\sum_{j}\left[\alpha_{j i}^{*} \bar{u}_{j}-\beta_{j i} \bar{u}_{j}^{*}\right]=\sum_{j}\left[\left(u_{i}, \bar{u}_{j}\right) \bar{u}_{j}-\left(u_{i}, \bar{u}_{j}^{*}\right) \bar{u}_{j}^{*}\right]
$$

em que usamos que $\alpha_{i j}^{*}=\left(\bar{u}_{i}, u_{j}\right)^{*}=\left(u_{j}, \bar{u}_{i}\right)$ e $\beta_{i j}=-\left(\bar{u}_{i}, u_{j}^{*}\right)=-\left(u_{j}, \bar{u}_{i}^{*}\right)$. Estes são os chamados coeficientes de Bogolubov e as transformações entre $u$ e $\bar{u}$ são chamadas de transformações de Bogolubov.

Para comparar os operadores de criação e de aniquilação vamos igualar as expressões para $\phi(x)$ obtendo:

$$
\phi(x)=\sum_{i}\left[a_{i} u_{i}(x)+a_{i}^{\dagger} u_{i}^{*}(x)\right]=\sum_{j}\left[\bar{a}_{j} \bar{u}_{j}(x)+\bar{a}_{j}^{\dagger} \bar{u}_{j}^{*}(x)\right] .
$$

Logo usando as relações de ortogonalidade chegamos à: 


$$
\begin{aligned}
& a_{i}=\sum_{j}\left[\bar{a}_{j}\left(\bar{u}_{j}, u_{i}\right)+\bar{a}_{j}^{*}\left(\bar{u}_{j}^{*}, u_{i}\right)\right]=\sum_{j}\left[\alpha_{j i} \bar{a}_{j}+\beta_{j i}^{*} \bar{a}_{j}^{*}\right] \\
& \bar{a}_{j}=\sum_{i}\left[a_{i}\left(u_{i}, \bar{u}_{j}\right)+a_{i}^{\dagger}\left(u_{i}^{*}, \bar{u}_{j}\right)\right]=\sum_{i}\left[\alpha_{j i}^{*} a_{i}-\beta_{j i}^{*} a_{i}^{\dagger}\right] .
\end{aligned}
$$

Estaremos interessados em saber a média de quantas partículas, $N_{i}$, no modo $u_{i}$ encontraremos no vácuo do espaço de Fock construído com o espaço de Hilbert das funções $\bar{u}_{i}$, denotado por $\mid \overline{0}>$. O operador que corresponde ao observável $N_{i}$, partículas no modo $u_{i}$, é chamado de $\hat{N}_{i}$ e é dado por $\hat{N}_{i}=a_{i}^{\dagger} a_{i}$. Assim queremos saber $<\overline{0}\left|\hat{N}_{i}\right| \overline{0}>=<\overline{0}\left|a_{i}^{\dagger} a_{i}\right| \overline{0}>$. Usando a relação 2.7 obtemos que $a_{i}\left|\overline{0}>=\sum_{j} \beta_{j i}^{*}\right| \overline{1}_{j}>$. Assim

$$
<\overline{0}\left|\hat{N}_{i}\right| \overline{0}>=\sum_{j}\left|\beta_{j i}\right|^{2} .
$$

Além destas relações, será usada mais outra relação na seção abaixo entre os coeficientes de Bogolubov. Esta relação pode ser expressa por:

$$
\sum_{k}\left(\alpha_{i k} \alpha_{j k}^{*}-\beta_{i k} \beta_{j k}^{*}\right)=\delta_{i j} .
$$

Esta relação pode ser provada usando a relação de comutação 2.6 e a relação 2.8 da seguinte forma:

$$
\begin{gathered}
\delta_{i j}=\left[\bar{a}_{i}, \bar{a}_{j}^{\dagger}\right]=\left[\sum_{k}\left(\alpha_{i k}^{*} a_{k}-\beta_{i k}^{*} a_{k}^{\dagger}\right), \sum_{l}\left(\alpha_{j l} a_{l}^{\dagger}-\beta_{j l} a_{l}\right)\right]= \\
\sum_{k, l} \alpha_{i k}^{*} \alpha_{j l}\left[a_{k}, a_{l}^{\dagger}\right]+\sum_{k, l} \beta_{i k}^{*} \beta_{j l}\left[a_{k}^{\dagger}, a_{l}\right]=\sum_{k, l} \alpha_{i k}^{*} \alpha_{j l} \delta_{k l}-\sum_{k, l} \beta_{i k}^{*} \beta_{j l} \delta_{k l}= \\
\sum_{k}\left(\alpha_{i k}^{*} \alpha_{j k}-\beta_{i k}^{*} \beta_{j k}\right) .
\end{gathered}
$$

Utilizando-se destes resultados, vamos aplicar agora a quantização acima no espaço de Rindler, a fim de saber como é visto o vácuo convencional (dos observadores inerciais) da teoria de campos construída no espaço de Minkowski por observadores uniformemente acelerados.

Antes, porém, vamos fazer alguns comentários sobre a forma como o campo foi quantizado. Usamos uma abordagem bastante pouco precisa do ponto de vista matemático. Ela se assemelha às construções inicialmente vistas em cursos de teoria de campos para físicos e na referência Birrel e Davies [16].

No entanto, existem abordagens mais cuidadosas para a construção de campos escalares lineares em variedades globalmente hiperbólicas que contenham superfícies de Cauchy infinitamente diferenciáveis, como foi feito por Dimock [3]. Apenas com o objetivo de ilustrar estas idéias, vamos fazer um apanhado delas abaixo baseados no artigo [3]. 


\subsubsection{A quantização canônica em espaços globalmente hiperbólicos: $O$ trabalho de Dimock}

A idéia deste trabalho e dos trabalhos rigorosos matematicamente que antecederam-no e sucederamno é, inicialmente, estudar a equação clássica do campo que estaremos quantizando. Como estamos interessados em estudar campos que satisfaçam a equação de Klein-Gordon, estudaremos a equação dada por:

$$
\left(\square+m^{2}\right) u=0
$$

em que

$$
\square=\nabla^{\nu} \nabla_{\nu}=|g|^{-\frac{1}{2}} \partial_{\mu} g^{\mu \nu}|g|^{\frac{1}{2}} \partial_{\nu}
$$

para $|g|=\left|\operatorname{det}\left\{g_{\mu \nu}\right\}\right|$ e $m$ sendo a massa.

Como conseqüência da hiperbolicidade global, prova-se a existência e unicidade de certos operadores $E^{ \pm}: C_{0}^{\infty}(M) \rightarrow C^{\infty}(M)$. Notamos que estamos definindo $C^{\infty}(M)$ como o conjunto das funções de $M$ em $\mathbb{R}$ que são infinitamente diferenciáveis e por $C_{0}^{\infty}$ as funções de $C^{\infty}(M)$ que possuem suporte compacto. Estes operadores são os únicos que satisfazem as relações:

$$
\begin{gathered}
\left(\square+m^{2}\right) E^{ \pm}=E^{ \pm}\left(\square+m^{2}\right)=I \\
\operatorname{supp}\left(E^{ \pm} f\right) \subset J^{ \pm}(\operatorname{supp} f) .
\end{gathered}
$$

As funções $E^{ \pm} f$ são também chamadas de soluções fundamentais avançadas e retardadas da equação de Klein-Gordon.

Para $A \subset M$ definimos $J^{+}(A):=\{x \in M$; existe uma curva causal tipo tempo dirigida para o futuro que liga $A$ a um ponto $x\}$. A definição de $J^{-}(A)$ é a mesma, porém a curva é dirigida para o passado. Dada uma superfície de Cauchy $S$ do espaço-tempo, também devemos definir os operadores $\rho_{0}: C^{\infty}(M) \rightarrow C^{\infty}(S)$ e $\rho_{1}: C^{\infty}(M) \rightarrow C^{\infty}(S)$ como os operadores de restrição de uma função a $S$ e derivada normal ao futuro, respectivamente. Uma vez que definimos estes operadores podemos enunciar o Teorema fundamental para o problema clássico, ou seja, sobre as soluções da equação diferencial:

Teorema 2.2.2.1 (Problema de CAuchy): Seja $S$ uma superficie de Cauchy infinitamente diferenciável e $u_{0}, u_{1} \in C_{0}^{\infty}(S)$. Então existe um único $u \in C^{\infty}(M)$ tal que $\left(\square+m^{2}\right) u=$ $0, \rho_{0}(u)=u_{0}, \rho_{1}(u)=u_{1}$. Além disto, supp $u \subset\left(\cup_{i} \cup_{ \pm} J^{ \pm}\left(\right.\right.$supp $\left.\left.u_{i}\right)\right)$. A solução será dada por:

$$
u=E \rho_{0}^{\prime} u_{1}-E \rho_{1}^{\prime} u_{0}
$$

em que $E=E^{+}-E^{-}$e $\rho_{0}^{\prime}$ e $\rho_{1}^{\prime}$ são os operadores adjuntos de $\rho_{0}$ e $\rho_{1}$. 
Notamos que se $\rho_{0}: C^{\infty}(M) \rightarrow C^{\infty}(S)$ então $\rho_{0}^{\prime}: C^{\infty}(S)^{\prime} \rightarrow C^{\infty}(M)^{\prime}$ é uma função entre os duais. Porém no dual de $C^{\infty}(S), C^{\infty}(S)^{\prime}$, existem as funções de $C_{0}^{\infty}(S)$, identificadas com o funcional integração: $f \in C^{\infty}(S) \rightarrow \int f g d S$ para $g \in C_{0}^{\infty}(S)$. Assim a função $\rho_{0}^{\prime}$ pode também ser pensada como uma função restrita ao domínio $C_{0}^{\infty}(S)$.

Uma vez que conhecemos a solução do problema clássico, podemos passar ao problema quântico. Este é resolvido definindo as álgebras de Weyl. Elas são funções de $C_{0}^{\infty}(S) \times C_{0}^{\infty}(S)$ nos operadores unitários em um espaço de Hilbert qualquer tal que $t \rightarrow W\left(t h, t h^{\prime}\right)$ é fortemente contínuo e tal que valha a relação

$$
W\left(h_{1}, h_{1}^{\prime}\right) W\left(h_{2}, h_{2}^{\prime}\right)=W\left(h_{1}+h_{2}, h_{1}^{\prime}+h_{2}^{\prime}\right) \exp \left(-\frac{i}{2}\left(<h_{1}, h_{2}^{\prime}>-<h_{2}, h_{1}^{\prime}>\right)\right)
$$

Na expressão acima usamos a notação $<h_{1}, h_{2}^{\prime}>=\int h_{1} h_{2}^{\prime} d S$.

Pode-se mostrar que a existência de um tal espaço de Hilbert que permita a definição das álgebras de Weyl sempre existe. Podemos definir o operador de campo $\phi(f), f \in C_{0}^{\infty}(M)$, como o único operador auto-adjunto que, de acordo com o Teorema de Stone [6], satisfaz a igualdade

$$
e^{i \phi(f) t}=W\left(t \rho_{1} E f, t \rho_{0} E f\right), \text { qualquer } t \in \mathbb{R} .
$$

Podemos, partindo destas definições, definir uma rede de álgebras em conjuntos relativamente compactos $\mathcal{O}$ por

$$
\mathcal{A}(\mathcal{O})=\text { álgebra } \mathrm{C}^{*} \text { gerada por } e^{i \phi(f)}=W\left(\rho_{1} E f, \rho_{0} E f\right), \operatorname{supp} f \subset \mathcal{O}
$$

e definir uma álgebra $C^{*}$ dos observáveis da teoria por

$$
\mathcal{A}=\overline{\cup_{\mathcal{O}} \mathcal{A}(\mathcal{O})}=\mathcal{A}(M)
$$

Por fim, com estas definições pode-se mostrar dois Teoremas fundamentais.

TeOrema 2.2.2.2: A rede $\mathcal{A}(\mathcal{O})$ é independente da representação da álgebra CCR definida anteriormente sobre um determinado espaço de Hilbert, assim como é independente da superfície de Cauchy.

Por independência queremos dizer que se temos duas álgebras $\mathcal{A}$ e $\mathcal{A}^{\prime}$. Então existe um isomorfismo $i: \mathcal{A} \rightarrow \mathcal{A}^{\prime}$ tal que $i[\mathcal{A}(\mathcal{O})]=\mathcal{A}^{\prime}(\mathcal{O})$. Isto é análogo ao fato da integral 2.4 não depender da superfície de Cauchy. Por fim, o resultado físico mais importante desta discussão é que a rede de álgebras construídas desta maneira satisfaz axiomas análogos aos de Haag-Kastler [22]. Estes axiomas se assemelham os axiomas de Wightmann que serão enunciados na próxima seção. Ambos os esquemas são bastante relacionados. 
TeOrema: 2.2.2.3 Seja um espaço-tempo globalmente hiperbólico e $\mathcal{A}(\mathcal{O})$ uma rede de observáveis locais definidos acima. Então $\mathcal{A}(\mathcal{O})$ satisfaz:

1. Para todo aberto limitado $\mathcal{O}$ em $M$, existe uma álgebra $C^{*} \mathcal{A}(\mathcal{O})$. Se $\mathcal{O} \subset \mathcal{O}^{\prime}$ então $\mathcal{A}(\mathcal{O}) \subset \mathcal{A}\left(\mathcal{O}^{\prime}\right)$. A álgebra $C^{*} \mathcal{A}=\overline{\cup_{\mathcal{O}} \mathcal{A}(\mathcal{O})}$ é chamada de álgebra dos observáveis.

2. Existe uma representação irredutível e fiel de $\mathcal{A}$.

3. Se $\mathcal{O}$ tem uma separação tipo espaço de $\mathcal{O}^{\prime}$, então $\left[\mathcal{A}(\mathcal{O}), \mathcal{A}\left(\mathcal{O}^{\prime}\right)\right]=0$.

4. Se $\mathcal{O}$ é causualmente dependente de $\mathcal{O}^{\prime}$ então $\mathcal{A}(\mathcal{O}) \subset \mathcal{A}\left(\mathcal{O}^{\prime}\right)$.

5. Para qualquer isometria entre espaços-tempos $\kappa:(M, g) \rightarrow\left(M^{\prime}, g^{\prime}\right)$, existe uma isometria $\alpha_{\kappa}: \mathcal{A} \rightarrow \mathcal{A}^{\prime}$ tal que $\alpha_{\kappa}[\mathcal{A}(\mathcal{O})]=\mathcal{A}^{\prime}(\kappa(\mathcal{O}))$. Além disto $\alpha_{i d}=i d$ e $\alpha_{\kappa_{1}} \circ \alpha_{\kappa_{2}}=\alpha_{\kappa_{1} \circ \kappa_{2}}$.

É bastante instrutivo saber como aparecem os operadores $\phi(t, \vec{x})$ e $\pi(t, \vec{x})$ mostrados anteriormente para o espaço de Minkowski. Usando as relações CCR, sabemos que, devido ao Teorema de Stone, existem operadores $\theta(h)$ e $\pi\left(h^{\prime}\right)$ tal que

$$
e^{i \theta(h) t}=W(t h, 0) \quad e^{-i \pi\left(h^{\prime}\right) t}=W\left(0, t h^{\prime}\right)
$$

Se escolhermos a superfície de Cauchy como sendo a superfície $t=0$, então estes operadores poderão ser pensados como

$$
\theta(h)=\int \phi(0, \vec{x}) h(\vec{x}) d^{3} \vec{x} \text { e } \pi\left(h^{\prime}\right)=\int \pi(0, \vec{x}) h^{\prime}(\vec{x}) d^{3} \vec{x}
$$

A relação de comutação será

$$
\left[\theta(h), \pi\left(h^{\prime}\right)\right]=i \int h h^{\prime} d S=i \int h h^{\prime} d^{3} \vec{x} .
$$

que é o análogo a equação 2.3. Assim estabelecemos a relação entre as duas formulações.

\subsubsection{O vácuo dos observadores inerciais visto por observadores uni- formemente acelerados}

Nesta seção, trataremos apenas com o espaço de Minkowski. Vamos nos restringir a espaços em $1+1$ dimensão, conveniente para nossos propósitos. Sua métrica nas coordenadas $\bar{u}=t-x$ e $\bar{v}=t+x$ podem ser escritas como:

$$
d s^{2}=d t^{2}-d x^{2}=d \bar{u} d \bar{v}
$$


Podemos, como já foi feito anteriormente no espaço de Rindler, parametrizá-lo por $\eta$ e $\xi$, ambos variando em $\mathbb{R}$, da seguinte forma:

$$
\begin{gathered}
t=\frac{1}{a} e^{a \xi} \sinh (a \eta) \\
x=\frac{1}{a} e^{a \xi} \cosh (a \eta) .
\end{gathered}
$$

Ou de forma equivalente

$$
\begin{gathered}
\bar{u}=-\frac{1}{a} e^{-a u} \\
\bar{v}=\frac{1}{a} e^{a v}
\end{gathered}
$$

em que $u=\eta-\xi$ e $v=\eta+\xi$. A métrica nestas coordenadas fica, então, $d s^{2}=e^{2 a \xi} d u d v=$ $e^{2 a \xi}\left(d \eta^{2}-d \xi^{2}\right)$. As linhas em que $\eta$ é constante são retas e definem superfícies de Cauchy. As linhas em que $\xi$ são constantes definem hipérboles que representam linhas-mundo de observadores uniformemente acelerados, cuja aceleração própria é $a e^{-a \xi}$. O tempo próprio dos observadores é $\tau=e^{a \xi} \eta$.

Com estas parametrizações gostaríamos de quantizar um campo escalar complexo sem massa. A equação de onda será:

$$
\square \phi \equiv\left(\frac{\partial^{2}}{\partial t^{2}}-\frac{\partial^{2}}{\partial x^{2}}\right) \phi \equiv \frac{\partial^{2} \phi}{\partial \bar{u} \partial \bar{v}}=0
$$

Esta equação possui as soluções ortonormais dadas por $\bar{u}_{k}=(4 \pi \omega)^{-\frac{1}{2}} e^{i k x-i \omega t}$ em que $\omega=$ $|k|>0$ e $k \in \mathbb{R}$. Estes modos são de freqüência positiva em relação ao vetor de Killing $\partial_{t}$, ou seja, satisfazem:

$$
\mathcal{L}_{\partial_{t}} \bar{u}_{k}=-i \omega \bar{u}_{k}
$$

Utilizamos acima a notação de $\mathcal{L}_{v}$ para a derivada de Lie em relação ao vetor $v$. A definição mais geral de derivada de Lie pode ser encontrada no livro de Robert Wald [33]. Para nós apenas importa saber que a derivada de Lie em relação a um vetor $v$ quando aplicada a uma função corresponde ao valor do vetor aplicado aquela função. A equação acima pode ser lida como:

$$
\mathcal{L}_{\partial_{t}} \bar{u}_{k}=\partial_{t} \bar{u}_{k}=-i \omega \bar{u}_{k} .
$$

A equação de onda nas variáveis $\eta$ e $\xi$, ou $u$ e $v$, será dada por:

$$
e^{2 a \xi} \square \phi=\left(\frac{\partial^{2}}{\partial \eta^{2}}-\frac{\partial^{2}}{\partial \xi^{2}}\right) \phi=\frac{\partial^{2} \phi}{\partial u \partial v}=0 .
$$


Para a qual existem soluções:

$$
u_{k}=\frac{1}{\sqrt{4 \pi \omega}} e^{i k \xi \pm i \omega \eta}
$$

em que $\omega=|k|$ para $k \in \mathbb{R}$. O sinal + se aplica na região $\mathrm{L}=\left\{(t, x) \in \mathbb{R}^{2} ; x<-|t|\right\}$ e o sinal - se aplica na região $\mathrm{R}=\left\{(t, x) \in \mathbb{R}^{2} ; x>|t|\right\}$. As soluções são de freqüência positiva com respeito ao vetor de Killing $\partial_{\eta}$ em $\mathrm{R}$ e $-\partial_{\eta}$ em L, satisfazendo:

$$
\mathcal{L}_{ \pm \partial_{\eta}} u_{k}=-i \omega u_{k}
$$

Assim, usando $\bar{u}_{k}$ obteremos o campo visto por observadores inerciais e usando $u_{k}$ obteremos o campo tal como visto por observadores uniformemente acelerados.

Desta forma podemos expandir a função de onda $\phi(t, x)$ de duas formas utilizando as diferentes soluções acima $u_{k}$ e $\bar{u}_{k}$. Estas expansões podem ser vistas de acordo com a equação 2.5 da seguinte forma:

$$
\hat{\phi}(t, x)=\int_{-\infty}^{\infty} \frac{d k}{(2 \pi)^{\frac{1}{2}}} \frac{1}{\sqrt{2|k|}}\left[e^{-i|k| t+i k x} \hat{a}_{k}^{-}+e^{i|k| t-i k x} \hat{a}_{k}^{+}\right] .
$$

Se expandirmos usando as funções $\bar{u}_{\vec{k}}$. Se o fizermos usando as funções $u_{\vec{k}}$ obtemos:

$$
\hat{\phi}(\eta, \xi)=\int_{-\infty}^{\infty} \frac{d k}{(2 \pi)^{\frac{1}{2}}} \frac{1}{\sqrt{2|k|}}\left[e^{-i|k| \eta+i k \xi} \hat{a}_{k}^{-}+e^{i|k| \eta-i k \xi} \hat{a}_{k}^{+}\right] .
$$

Seguiremos agora [20] a fim de conhecer os coeficientes de Bogolubov que relacionam estas duas quantizações. A idéia do procedimento será notar que, pelas equações 2.11 e 2.12, concluímos que $\bar{u}$ é apenas função de $u$ e $\bar{v}$ é apenas função de $v$. Assim, escrevendo as equações 2.13 e 2.14 em função destas variáveis e fazendo com que os termos exponenciais contenham apenas um destes termos, poderemos calcular mais facilmente os coeficientes de Bogolubov.

Para que se possa entender melhor o que foi dito acima, vamos inicialmente escrever as fórmulas das expansões de onda em termos de $u, v, \bar{u}$ e $\bar{v}$ e de forma que as exponenciais contenham apenas um destes termos. Vamos fazer os cálculos explicitamente para as expansão 2.13. Os cálculos para 2.14 são exatamente os mesmos. O procedimento é o seguinte:

$$
\begin{gathered}
\hat{\phi}(t, x)=\int_{-\infty}^{\infty} \frac{d k}{(2 \pi)^{\frac{1}{2}}} \frac{1}{\sqrt{2|k|}}\left[e^{-i|k| t+i k x} \hat{a}_{k}^{-}+e^{i|k| t-i k x} \hat{a}_{k}^{+}\right]= \\
=\int_{-\infty}^{0} \frac{d k}{(2 \pi)^{\frac{1}{2}}} \frac{1}{\sqrt{2|k|}}\left[e^{-i|k| t+i k x} \hat{a}_{k}^{-}+e^{i|k| t-i k x} \hat{a}_{k}^{+}\right]+\int_{0}^{\infty} \frac{d k}{(2 \pi)^{\frac{1}{2}}} \frac{1}{\sqrt{2|k|}}\left[e^{-i|k| t+i k x} \hat{a}_{k}^{-}+e^{i|k| t-i k x} \hat{a}_{k}^{+}\right] \\
=\int_{0}^{\infty} \frac{d \omega}{(2 \pi)^{\frac{1}{2}}} \frac{1}{\sqrt{2 \omega}}\left[e^{-i \omega(t+x)} \hat{a}_{-\omega}^{-}+e^{i \omega(t+x)} \hat{a}_{-\omega}^{+}\right]+\int_{0}^{\infty} \frac{d \omega}{(2 \pi)^{\frac{1}{2}}} \frac{1}{\sqrt{2 \omega}}\left[e^{-i \omega(t-x)} \hat{a}_{\omega}^{-}+e^{i \omega(t-x)} \hat{a}_{\omega}^{+}\right] .
\end{gathered}
$$


Agora basta usar a definição de $\bar{u}$ e $\bar{v}$ e concluímos então que:

$$
\hat{\phi}(t, x)=\int_{0}^{\infty} \frac{d \omega}{(2 \pi)^{\frac{1}{2}}} \frac{1}{\sqrt{2 \omega}}\left\{\left[e^{-i \omega \bar{v}} \hat{a}_{-\omega}^{-}+e^{i \omega \bar{v}} \hat{a}_{-\omega}^{+}\right]+\left[e^{-i \omega \bar{u}} \hat{a}_{\omega}^{-}+e^{i \omega \bar{u}} \hat{a}_{\omega}^{+}\right]\right\} .
$$

Prosseguindo exatamente da mesma forma, podemos expressar a equação 2.14 em termos de $u$ e $v$ da forma

$$
\hat{\phi}(\eta, \xi)=\int_{0}^{\infty} \frac{d \Omega}{(2 \pi)^{\frac{1}{2}}} \frac{1}{\sqrt{2 \Omega}}\left\{\left[e^{-i \Omega v} \hat{b}_{-\Omega}^{-}+e^{i \Omega v} \hat{b}_{-\Omega}^{+}\right]+\left[e^{-i \Omega u} \hat{b}_{\Omega}^{-}+e^{i \Omega u} \hat{b}_{\Omega}^{+}\right]\right\} .
$$

Uma vez que conseguimos expressar a função de onda com termos contendo apenas $u, v, \bar{u}$ ou $\bar{v}$, podemos usar o fato de que $u$ é função apenas de $\bar{u}$ e $v$ é apenas função de $\bar{v}$ para então concluirmos que:

$$
\begin{gathered}
\int_{0}^{\infty} \frac{d \omega}{(2 \pi)^{\frac{1}{2}}} \frac{1}{\sqrt{2 \omega}}\left\{\left[e^{-i \omega \bar{v}} \hat{a}_{-\omega}^{-}+e^{i \omega \bar{v}} \hat{a}_{-\omega}^{+}\right]=\int_{0}^{\infty} \frac{d \Omega}{(2 \pi)^{\frac{1}{2}}} \frac{1}{\sqrt{2 \Omega}}\left\{\left[e^{-i \Omega v} \hat{b}_{-\Omega}^{-}+e^{i \Omega v} \hat{b}_{-\Omega}^{+}\right],\right.\right. \\
\int_{0}^{\infty} \frac{d \omega}{(2 \pi)^{\frac{1}{2}}} \frac{1}{\sqrt{2 \omega}}\left[e^{-i \omega \bar{u}} \hat{a}_{\omega}^{-}+e^{i \omega \bar{u}} \hat{a}_{\omega}^{+}\right]=\int_{0}^{\infty} \frac{d \Omega}{(2 \pi)^{\frac{1}{2}}} \frac{1}{\sqrt{2 \Omega}}\left[e^{-i \Omega u} \hat{b}_{\Omega}^{-}+e^{i \Omega u} \hat{b}_{\Omega}^{+}\right] .
\end{gathered}
$$

Para extrair enfim os coeficientes de Bogolubov vamos analisar a equação 2.16. Fazendo a transformada de Fourier em ambos os lados obtemos do lado direito:

$$
\begin{gathered}
\int_{-\infty}^{\infty} \frac{d u}{\sqrt{2 \pi}} e^{i x u} \int_{0}^{\infty} \frac{d \Omega}{(2 \pi)^{\frac{1}{2}}} \frac{1}{\sqrt{2 \Omega}}\left[e^{-i \Omega u} \hat{b}_{\Omega}^{-}+e^{i \Omega u} \hat{b}_{\Omega}^{+}\right]=\int_{0}^{\infty} \frac{d \Omega}{2 \pi} \frac{1}{\sqrt{2 \Omega}} \int_{-\infty}^{\infty} d u\left[e^{-i(\Omega-x) u} \hat{b}_{\Omega}^{-}+e^{i(\Omega+x) u} \hat{b}_{\Omega}^{+}\right] \\
=\int_{0}^{\infty} \frac{d \Omega}{\sqrt{2 \Omega}}\left[\delta(\Omega-x) \hat{b}_{\Omega}^{-}+\delta(\Omega+x) \hat{b}_{\Omega}^{+}\right]=\frac{1}{\sqrt{2|x|}}\left\{\begin{array}{c}
\hat{b}_{x}^{-}, x>0 \\
\hat{b}_{|x|}^{+}, x<0
\end{array} .\right.
\end{gathered}
$$

O lado esquerdo por sua vez fica:

$$
\begin{gathered}
\int_{-\infty}^{\infty} \frac{d u}{\sqrt{2 \pi}} e^{i x u} \int_{0}^{\infty} \frac{d \omega}{(2 \pi)^{\frac{1}{2}}} \frac{1}{\sqrt{2 \omega}}\left[e^{-i \omega \bar{u}} \hat{a}_{\omega}^{-}+e^{i \omega \bar{u}} \hat{a}_{\omega}^{+}\right]=\int_{0}^{\infty} \frac{d \omega}{\sqrt{2 \omega}} \int_{-\infty}^{\infty} \frac{d u}{2 \pi}\left[e^{i x u-i \omega \bar{u}} \hat{a}_{\omega}^{-}+e^{i x u+i \omega \bar{u}} \hat{a}_{\omega}^{+}\right] \\
=\int_{0}^{\infty} \frac{d \omega}{\sqrt{2 \omega}}\left[F(\omega, \Omega) \hat{a}_{\omega}^{-}+F(-\omega, \Omega) \hat{a}_{\omega}^{+}\right] .
\end{gathered}
$$

Acima, definimos

$$
F(\omega, \Omega):=\int_{-\infty}^{\infty} \frac{d u}{2 \pi} e^{i \Omega u-i \omega \bar{u}}
$$

Lembrando que $\bar{u}=-\frac{1}{a} e^{-a u}$, obtemos que $F(\omega, \Omega)$ também pode ser expresso por: 


$$
F(\omega, \Omega)=\int_{-\infty}^{\infty} \frac{d u}{2 \pi} e^{i \Omega u+i \frac{\omega}{a} e^{-a u}}
$$

Estas integrais devem ser entendidas no sentido de distribuição, ou seja, $F(\omega, \Omega)$ deve ser entendido como um funcional linear em $\mathcal{D}\left(\mathbb{R}^{2}\right)$ que associa a cada $f \in \mathcal{D}\left(\mathbb{R}^{2}\right)$ o valor $\int_{-\infty}^{\infty} \frac{d u}{2 \pi}\left\{\int e^{i \Omega u+i \frac{\omega}{a} e^{-a u}} f(\omega, \Omega) d \omega d \Omega\right\} \in \mathbb{C}$.

Assim, pelas equações acima, concluímos que os operadores $\hat{b}_{\Omega}^{-}$podem ser expressos em termos dos coeficientes de Bogolubov $\alpha_{\omega \Omega}$ e $\beta_{\omega \Omega}$ tais como em 2.7. Usando as relações anteriores obtemos:

$$
\hat{b}_{\Omega}^{-}=\int_{0}^{\infty} d \omega\left[\alpha_{\omega \Omega} \hat{a}_{\omega}^{-}+\beta_{\omega \Omega} \hat{a}_{\omega}^{+}\right]=\sqrt{\frac{\Omega}{\omega}} \int_{0}^{\infty} d \omega\left[F(\omega, \Omega) \hat{a}_{\omega}^{-}+F(-\omega, \Omega) \hat{a}_{\omega}^{+}\right]
$$

Em especial obtemos que $\beta_{\omega \Omega}=\sqrt{\frac{\Omega}{\omega}} F(-\omega, \Omega)$. Este resultado é particularmente interessante, pois se quisermos saber quantas partículas em média no modo $\Omega$ existem no vácuo de Minkowski basta usar o resultado 2.9 e obter:

$$
N_{\Omega}=\int_{0}^{\infty} d \omega\left|\beta_{\omega \Omega}\right|^{2}=\int_{0}^{\infty} d \omega \frac{\Omega}{\omega} F^{*}(-\omega, \Omega) F(-\omega, \Omega) .
$$

Para calcular este valor usaremos a relação:

$$
F(\omega, \Omega)=F(-\omega, \Omega) \exp \left(\frac{\pi \Omega}{a}\right)
$$

e a relação:

$$
\int d \omega\left(\alpha_{\omega \Omega} \alpha_{\omega \Omega^{\prime}}^{*}-\beta_{\omega \Omega} \beta_{\omega \Omega^{\prime}}^{*}\right)=\delta\left(\Omega-\Omega^{\prime}\right)
$$

que é obtida da mesma forma que 2.10, porém usando as relações $\left[\hat{a}_{\omega}^{-}, \hat{a}_{\omega^{\prime}}^{+}\right]=\delta\left(\omega-\omega^{\prime}\right)$ e análogas para $\hat{b}$, que por sua vez são as mesmas de 2.6, porém para funções de onda não normalizadas. Utilizando a equação acima, obtemos a seguinte relação:

$$
\begin{gathered}
\int_{0}^{\infty} d \omega \frac{\sqrt{\Omega \Omega^{\prime}}}{\omega}\left[F(\omega, \Omega) F^{*}\left(\omega, \Omega^{\prime}\right)-F(-\omega, \Omega) F^{*}\left(-\omega, \Omega^{\prime}\right)\right]= \\
=\left[\exp \left(\frac{\pi\left(\Omega+\Omega^{\prime}\right)}{a}\right)-1\right] \int_{0}^{\infty} d \omega \frac{\sqrt{\Omega \Omega^{\prime}}}{\omega} F(-\omega, \Omega) F^{*}\left(-\omega, \Omega^{\prime}\right)=\delta\left(\Omega-\Omega^{\prime}\right)
\end{gathered}
$$

em que na igualdade utilizou-se a equação 2.17. Obtemos assim o resultado previsto pelo efeito Unruh:

$$
N_{\Omega}=\int_{0}^{\infty} d \omega\left|\beta_{\omega \Omega}\right|^{2}=\int_{0}^{\infty} d \omega \frac{\Omega}{\omega}|F(-\omega, \Omega)|^{2}=\frac{\delta(0)}{\exp \left(\frac{2 \pi \Omega}{a}\right)-1} .
$$

O termo $\delta(0)$ evidentemente não faz sentido e aparece porque usamos funções de onda não 
normalizadas. Ele deve ser interpretado como o volume. Assim o número de partículas por volume é:

$$
n_{\Omega}=\frac{1}{\exp \left(\frac{2 \pi \Omega}{a}\right)-1}
$$

Este resultado é idêntico ao valor esperado de partículas bosônicas de freqüência $\Omega$ imersas num banho térmico de temperatura $T=\frac{\hbar a}{2 \pi k c}$. Este, enfim, é o efeito Unruh.

Por fim devemos justificar a equação 2.17. Novamente seguiremos [20]. Para justificar esta relação, basta mostrar que podemos mudar o contorno para $u \rightarrow u-\frac{i \pi}{a}$ e então teremos:

$$
\begin{gathered}
F(\omega, \Omega)=\int_{-\infty}^{\infty} \frac{d u}{2 \pi} e^{i \Omega u+i \frac{\omega}{a} e^{-a u}} \rightarrow \int_{-\infty}^{\infty} \frac{d u}{2 \pi} e^{i \Omega\left(u-\frac{i \pi}{a}\right)+i \frac{\omega}{a} e^{-a\left(u-\frac{i \pi}{a}\right)}}= \\
=\int_{-\infty}^{\infty} \frac{d u}{2 \pi} e^{i \Omega u+\frac{\Omega \pi}{a}+i \frac{\omega}{a} e^{-a u} e^{+i \pi}}=\int_{-\infty}^{\infty} \frac{d u}{2 \pi} e^{i \Omega u+\frac{\Omega \pi}{a}-i \frac{\omega}{a} e^{-a u}}=F(-\omega, \Omega) e^{\frac{\Omega \pi}{a}}
\end{gathered}
$$

Para justificar a última igualdade, basta notar que nos caminhos $\gamma:\left[0, \frac{\pi}{a}\right] \rightarrow \mathbb{C}$ dado por $\gamma(t)=u-i t$, temos que o integrando vai a zero quando $|u| \rightarrow \infty$. Para $u \rightarrow-\infty$ isto pode ser visto da forma:

$$
e^{i \Omega(u-i t)+i \frac{\omega}{a} e^{-a(u-i t)}}=e^{i \Omega u} e^{\Omega t} e^{i \frac{\omega}{a} e^{-a u} \cos (a t)} e^{-\frac{\omega}{a} e^{-a u} \sin (a t)} .
$$

Vemos que o último termo vai a zero quando $u \rightarrow-\infty$, já que $\sin (a t)$ é positivo. Os outros termos são limitados, de forma que de fato o integrando se anula. Para o caso $u \rightarrow \infty$ o integrando não converge. Porém $F(\omega, \Omega)$ está sendo entendida no sentido de distribuição. Logo podemos pensar em $F(\omega, \Omega)$ como:

$$
F(\omega, \Omega)=\lim _{b \rightarrow 0^{+}} \int_{-\infty}^{\infty} \frac{d u}{2 \pi} \exp \left(-b u^{2}+i \Omega u+i \frac{\omega}{a} e^{-a u}\right)
$$

no sentido de limite de distribuições, ou seja, dado $f \in \mathcal{D}\left(\mathbb{R}^{2}\right), f(\omega, \Omega)$, temos:

$$
\begin{gathered}
\int_{-\infty}^{\infty} \frac{d u}{2 \pi}\left[\int f(\omega, \Omega) \exp \left(i \Omega u+i \frac{\omega}{a} e^{-a u}\right) d \omega d \Omega\right]= \\
\lim _{b \rightarrow 0^{+}} \int_{-\infty}^{\infty} \frac{d u}{2 \pi}\left[\int f(\omega, \Omega) \exp \left(-b u^{2}+i \Omega u+i \frac{\omega}{a} e^{-a u}\right) d \omega d \Omega\right] .
\end{gathered}
$$

Escrita desta forma o fator $-b u^{2}$ se torna $-b u^{2}+2 i b u t+2 b t^{2}$ o que faz com que o integrando convirja quando $u$ for ao infinito. 


\section{Capítulo 3}

\section{O efeito Unruh: ponto de vista algébrico}

Neste capítulo, vamos estudar inicialmente os resultados que julgamos mais fundamentais a respeito dos estados KMS. Temos com isto o objetivo de saber como fazer uma caracterização matematicamente precisa dos estados de equilíbrio. Faremos também um breve resumo acerca da formulação axiomática da teoria de campos, em particular dos axiomas de Wightman e de Osterwalder-Schrader. Temos com isto um duplo objetivo: Primeiro gostaríamos de mostrar o arcabouço teórico para compreender o trabalho de Sewell [27] e sua explicação do efeito Unruh, que será mostrado ao final deste capítulo. Segundo, também gostaríamos de fornecer material que acreditamos ser essencial para a compreensão do trabalho de Klein-Landau e que nos permitirá traçar uma nova estratégia para a demonstração do efeito Unruh.

Antes de tudo isto, faremos um breve apanhado do que acreditamos ser um conjunto mínimo necessário de definições acerca de álgebra de operadores para a realização deste trabalho.

\subsection{Propriedades fundamentais das álgebras de operadores}

Vamos agora apresentar alguns aspectos básicos de álgebra de operadores que irão aparecer neste trabalho. A apresentação será breve e não daremos todas as demonstrações. Gostaríamos apenas de apresentar o que julgamos ser um mínimo para a compreensão do trabalho. Todos os resultados, sem excessão, podem ser encontrados nos livros do Kadison e Ringrose [14, 13]. Outra referência bastante conhecida são os livros de Bratteli e Robinson [8].

Inicialmente devemos definir uma álgebra $C^{*}$. Álgebras $C^{*}$ são cruciais neste trabalho por representarem os observáveis do sistemas físicos estudados. Vamos apenas estudar álgebras $C^{*}$ com unidade neste trabalho.

Para defini-las, é conveniente sabermos a princípio o que é uma álgebra de Banach.

DEFINIÇÃo 3.1.1: Uma álgebra $\mathcal{A}$ é uma álgebra de Banach se for associativa, com unidade $I$, normada e completa na métrica dada pela norma. Esta norma deve satisfazer $\|A B\| \leq$ $\|A\|\|B\|$, para todo $A$ e $B$ em $\mathcal{A}$, e $\|I\|=1$. 
Para definir corretamente uma álgebra $C^{*}$, é preciso, antes de mais nada, definir uma operação de involução em álgebras da seguinte forma.

Definição 3.1.2: Uma operação de involução numa álgebra $\mathcal{A}$ é uma operação ${ }^{*}: \mathcal{A} \rightarrow \mathcal{A}$ que satisfaz para todo $S$ e $T$ em $\mathcal{A}$, e todo $\alpha$ e $\beta$ em $\mathbb{C}$ :

- $(\alpha S+\beta T)^{*}=\bar{\alpha} S^{*}+\bar{\beta} T^{*}$.

- $(S T)^{*}=T^{*} S^{*}$.

- $\left(T^{*}\right)^{*}=T$.

Dada estas definições, podemos definir uma álgebra $C^{*}$ :

DeFinição 3.1.3: Uma álgebra de Banach-* $\mathcal{A}$ é uma álgebra de Banach, em que está definida uma operação de involução com a propriedade de que $\left\|A^{*}\right\|=\|A\|$ para qualquer $A \in \mathcal{A}$. Uma álgebra $C^{*}$ é uma álgebra de Banach- ${ }^{*}$ com a propriedade $\left\|A^{*} A\right\|=\|A\|^{2}$ para qualquer A contido na álgebra.

Devemos notar que a propriedade $\left\|A^{*} A\right\|=\|A\|^{2}$ implica em $\left\|A^{*}\right\|=\|A\|$. Isto pode ser visto pela relação $\|A\|^{2}=\left\|A^{*} A\right\| \leq\left\|A^{*}\right\|\|A\|$, ou seja, $\|A\| \leq\left\|A^{*}\right\|$. Trocando $A$ por $A^{*}$ obtemos $\|A\|=\left\|A^{*}\right\|$. É importante também que se perceba que a propriedade $\left\|A^{*} A\right\|=\|A\|^{2}$ é inspirada nas propriedades de operadores limitados agindo num espaço de Hilbert.

De fato, o exemplo mais imediato de uma álgebra $C^{*}$ é a álgebra dos operadores limitados agindo em um espaço de Hilbert, $\mathcal{H}$, denotada por $\mathcal{B}(\mathcal{H})$, com a operação de tomar o adjunto do operador no papel de involução. Como veremos, essencialmente toda álgebra $C^{*}$ pode ser vista desta forma. Para sabermos em que sentido esta afirmação é verdadeira, vamos definir uma representação de uma álgebra $C^{*}$ da seguinte forma:

Definição 3.1.4: Uma representação de uma álgebra $C^{*}, \mathcal{A}$, em um espaço de Hilbert $\mathcal{H}$ é uma função $\pi: \mathcal{A} \rightarrow \mathcal{B}(\mathcal{H})$ tal que para todo $S$ e $T$ em $\mathcal{A}$, e todo $\alpha$ e $\beta$ em $\mathbb{C}$

- $\pi(\alpha S+\beta T)=\alpha \pi(S)+\beta \pi(T)$.

- $\pi(S T)=\pi(S) \pi(T)$.

- $\pi\left(A^{*}\right)=\pi(A)^{*}$.

Dizemos que uma representação é fiel se ela for injetora.

Com a noção exata do que é uma representação, podemos dar um sentido mais preciso quando dizemos que "no fundo toda álgebra $C^{*}$ é uma álgebra de operadores agindo num 
espaço de Hilbert". A afirmação precisa pode ser vista no seguinte Teorema devido a Gelfand e Naimark.

TeOrema (Gelfand-Naimark) 3.1.5: Toda álgebra $C^{*}$ tem uma representação fiel.

Logo após a introdução destas álgebras, é importante definirmos estados. Os estados definidos abaixo representarão os estados físicos da teoria assim como a álgebra representa os observáveis, daí obviamente vem o nome.

DeFinição 3.1.6: Um estado é um funcional linear numa álgebra $C^{*}, \mathcal{A}$, tal que para qualquer $A \in \mathcal{A}$ temos

- $\rho(A) \geq 0$ se $A$ é positivo.

- $\rho(I)=1$.

Pode-se provar que um funcional com estas caracteristicas é necessariamente contínuo, com norma 1 e hermiteano, ou seja, $\rho\left(A^{*}\right)=\overline{\rho(A)}$. Dizemos que o estado é fiel se $\rho(A)=0$ e $A$ é positivo implique que $A=0$.

Uma vez que sabemos o que é um estado, podemos utilizá-lo a fim de construir uma representação da álgebra num espaço de Hilbert. Uma construção bastante importante é a chamada construção GNS. Ela pode ser enunciada da seguinte forma:

TeOrema (Construção GNS) 3.1.7: Se $\rho$ é um estado de uma álgebra $C^{*}$, $\mathcal{A}$, existe uma representação $\pi_{\rho}$ de $\mathcal{A}$ em um espaço de Hilbert $\mathcal{H}_{\rho}$ e um vetor unitário $x_{\rho}$ tal que para qualquer $A \in \mathcal{A}$ temos:

$$
\rho(A)=\left\langle x_{\rho}, \pi_{\rho}(A) x_{\rho}\right\rangle,
$$

e tal que a varredura dos vetores da forma $\pi(A) x_{p}$, para qualquer $A \in \mathcal{A}$, é densa no espaço $\mathcal{H}_{p}$ (dizemos, então que a representação é cíclica em relação a $x_{p}$ ).

Além disto, se existe uma representação cíclica em relação a um vetor $x$, denotada por $\pi$, de $\mathcal{A}$ em um espaço de Hilbert $\mathcal{H}$ tal que a relação acima valha para um vetor cíclico $x$, então existe um isomorfismo sobrejetor $U: \mathcal{H}_{\rho} \rightarrow \mathcal{H}$ tal que

$$
x=U x_{\rho} \quad \pi(A)=U \pi_{\rho}(A) U^{*}(\forall A \in \mathcal{A}) .
$$

Um tipo mais específico de álgebra $C^{*}$ também bastante utilizado em física são as chamadas álgebras de von Neumann. Para seu estudo é conveniente que façamos algumas recordações. Devemos lembrar que dado um espaço de Hilbert, $\mathcal{H}$, podemos introduzir outras topologias 
localmente convexas em $\mathcal{B}(\mathcal{H})$ além daquela gerada pela norma. Iremos introduzir duas delas que nos bastarão para este trabalho. Elas são a topologia fraca e forte dos operadores definidas abaixo:

DeFiniÇÃo 3.1.8: A topologia fraca dos operadores é a topologia induzida em $\mathcal{B}(\mathcal{H})$ pelos funcionais lineares $\omega_{x, y}(A)=<A x, y>$ para qualquer que seja $A \in \mathcal{B}(\mathcal{H})$ e $x, y \in \mathcal{H}$. Uma base de vizinhanças desta topologia é dada pelos conjuntos:

$$
V\left(T_{0}: \omega_{x_{1}, y_{1}}, \ldots, \omega_{x_{m}, y_{m}} ; \epsilon\right)=\left\{T \in \mathcal{B}(\mathcal{H}) ;\left|<\left(T-T_{0}\right) x_{j}, y_{j}>\right|<\epsilon \text { para } \mathrm{j}=1, \ldots, \mathrm{m}\right\}
$$

DEFInIÇÃo 3.1.9: A topologia forte dos operadores é a topologia induzida em $\mathcal{B}(\mathcal{H})$ pelas semi-normas $p_{x}(T)=\|T x\|$ para qualquer que seja $T \in \mathcal{B}(\mathcal{H})$ e $x \in \mathcal{H}$. Uma base de vizinhanças desta topologia é dada pelos conjuntos:

$$
V\left(T_{0}: x_{1}, \ldots, x_{m} ; \epsilon\right)=\left\{T \in \mathcal{B}(\mathcal{H}) ;\left\|\left(T-T_{0}\right) x_{j}\right\|<\epsilon \text { para } \mathrm{j}=1, \ldots, \mathrm{m}\right\}
$$

Podemos agora definir uma álgebra de von Neumann como:

DefiniçÃo 3.1.10: Uma álgebra de von Neumann, $\mathcal{M}$, agindo no espaço de Hilbert $\mathcal{H}$ é um conjunto de operadores limitados de $\mathcal{H}$, ou seja, $\mathcal{M} \subset \mathcal{B}(\mathcal{H})$, tal que $\mathcal{M}$ é uma álgebra $C^{*}$ e é um conjunto fechado na topologia fraca dos operadores.

Note que poderíamos ter definido como sendo fechado na topologia forte também devido ao seguinte fato.

Proposição 3.1.11: O fecho nas topologias fraca e forte dos operadores coincide em um subconjunto convexo de $B(\mathcal{H})$. Particularmente em uma álgebra $C^{*}$.

Por fim, notemos que existe uma outra caracterização de álgebras de von Neumann dada pelo Teorema do duplo comutante:

Teorema (Do Duplo COMUtante) 3.1.12: Se $\mathcal{A}$ é uma álgebra auto-adjunta de operadores contendo o operador identidade e agindo no espaço de Hilbert $\mathcal{H}$, em especial se for uma álgebra $C^{*}$, então as topologias fraca e forte dos operadores coincidem com $\mathcal{A}^{\prime \prime}$ (em que $\mathcal{A}^{\prime}$ é o conjunto de todos operadores em $\mathcal{B}(\mathcal{H})$ que comutam com $\mathcal{A}$ e $\mathcal{A}^{\prime \prime}$ é o conjunto dos operadores em $\mathcal{B}(\mathcal{H})$ que comutam com $\left.\mathcal{A}^{\prime}\right)$.

Dado um sistema físico, muitas vezes restringe-se como os estados físicos acessíveis os estados normais. Esta é uma definição importante e será feita a seguir. 
DEFINIÇÃo 3.1.13: Um estado $\omega$ de uma álgebra $C^{*}$ é normal se dada uma rede de operadores crescente $\left\{A_{\alpha}\right\}$, porém limitada, então vale que

$$
\rho\left(\sup _{\alpha}\left(A_{\alpha}\right)\right)=\sup _{\alpha} \rho\left(A_{\alpha}\right)
$$

Existem caracterizações equivalentes de estados normais agindo em uma álgebra de von Neumann, como pode ser visto através do seguinte Teorema.

TEOREMA 3.1.14: As seguintes condições de um estado $\rho$ de uma álgebra de von Neumann $\mathcal{M}$ agindo em um espaço de Hilbert $\mathcal{H}$ são equivalentes:

1. $\rho=\sum_{n=1}^{\infty} \omega_{y_{n}}$, em que $\omega_{y_{n}}(A)=<y_{n}, A y_{n}>e \sum_{n=1}^{\infty}\left\|y_{n}\right\|^{2}=1$ com $\left\{y_{n}\right\}_{n \in \mathbb{N}}$ um conjunto ortogonal.

2. $\rho=\sum_{n=1}^{\infty} \omega_{x_{n}}$, em que $\omega_{x_{n}}(A)=<x_{n}, A x_{n}>$ e $\sum_{n=1}^{\infty}\left\|x_{n}\right\|^{2}=1$.

3. $\rho$ é contínuo na topologia fraca dos operadores na bola unitária de $\mathcal{M}$.

4. $\rho$ é contínuo na topologia forte dos operadores na bola unitária de $\mathcal{M}$.

5. $\rho$ é normal.

6. $\rho$ é um estado completamente aditivo, ou seja, dada uma sequência ortogonal de projetores $\left\{E_{\alpha}\right\}$ em $\mathcal{M}$, vale a relação $\rho\left(\sum_{\alpha} E_{\alpha}\right)=\sum_{\alpha} \rho\left(E_{\alpha}\right)$.

Para a descrição de um sistema físico ainda falta definir um grupo uniparamétrico de automorfismos. Este grupo descreverá a dinâmica do sistema e é definido por:

DEFINIÇÃO 3.1.15: Um grupo uniparamétrico de *automorfismos é uma conjunto de funções $\alpha_{t}: \mathcal{A} \rightarrow \mathcal{A}$ indexadas pelos reais tais que cada função satisfaz, para todo $\beta, \gamma \in \mathbb{C} e$ todo $S, T \in \mathcal{A}$ :

1. $\alpha_{t}(\beta S+\gamma T)=\beta \alpha_{t}(S)+\gamma \alpha_{t}(T)$.

2. $\alpha_{t}(S T)=\alpha_{t}(S) \alpha_{t}(T)$.

3. $\alpha_{t}\left(S^{*}\right)=\alpha_{t}(S)^{*}$.

Além disto estas funções satisfazem $\alpha_{t} \circ \alpha_{s}=\alpha_{t+s}$.

Estas definições e Teoremas, embora mostrados de forma extremamente sucinta nesta seção, correspondem ao básico para a compreensão do que se seguirá. 


\subsection{Estados KMS}

Durante este trabalho, nossa principal motivação será mostrar que certos estados são estados de equilíbrio termodinâmico. Para tanto, é crucial que saibamos como caracterizar um estado de equilíbrio e quais são os principais resultados acerca desta caracterização.

Para estudar estados de equilíbrio, vamos utilizar a formulação algébrica da mecânica estatística quântica (tal qual desenvolvido em Bratteli e Robinson [8]). Os observáveis serão representados por uma álgebra $C^{*}$ e os estados físicos pelos estados desta álgebra. A dinâmica do sistema é descrita por um grupo uniparamétrico de ${ }^{*}$-automorfismos $\alpha_{t}$ agindo nesta álgebra $C^{*}$. Partindo destes ingredientes, gostaríamos de saber como se caracteriza-se um estados de equilíbrio. Para isto vamos utilizar os chamados estados KMS (tal qual feito por Haag e outros [26]).

A fim de motivar nossa discussão, partamos de alguns exemplos. Sabemos que as caracterizações mais comuns de estados de equilíbrio usam os ensembles microcanônico, canônico e grande canônico. As variáveis termodinâmicas fixas em cada um destes ensembles são respectivamente a energia e o número de partículas, a temperatura e o número de partículas, a temperatura e o potencial químico. Algebricamente, a descrição grande canônica é a mais conveniente.

Na descrição grande canônica, temos um espaço de Hilbert $\mathcal{H}$ e operadores $H$ e $N$ autoadjuntos (não limitados em geral) representando a energia e o número de partículas. O estado de equilíbrio grande canônico é representado por um funcional agindo num elemento $A \in \mathcal{B}(\mathcal{H})$ por:

$$
\omega_{\beta, \mu}(A)=\frac{\operatorname{Tr}\left(e^{-\beta K} A\right)}{\operatorname{Tr}\left(e^{-\beta K}\right)}
$$

em que $K=H-\mu N$, no qual $\mu$ é o potencial químico e $\beta=\frac{1}{k T}$, sendo $k$ a constante de Boltzmann e $T$ a temperatura, consideradas constantes neste ensemble. Se definirmos a evolução temporal generalizada por

$$
A \in \mathcal{B}(\mathcal{H}) \rightarrow \tau_{t}(A)=e^{i K t} A e^{-i K t} \in \mathcal{B}(\mathcal{H})
$$

temos que o estado $\omega_{\beta, \mu}$ é um estado KMS. Este estado pode ser definido como:

DeFINiÇÃo 3.2.1: Dizemos que um grupo uniparamétrico $\left\{\tau_{t}\right\}$ de *automorfismos de uma álgebra de von Neumann $\mathcal{M}$ satisfaz a condição KMS em relação a um estado $\omega$ (chamado de estado KMS) se dados dois elementos $A$ e $B$ de $\mathcal{M}$ existe uma função complexa limitada, $F_{A, B}(z)$, contínua na faixa $\{z \in \mathbb{C}: 0 \leq \operatorname{Im} z \leq \beta\}$ e analítica no interior dela, tal que

$$
F_{A, B}(t)=\omega\left(\tau_{t}(A) B\right) \quad \text { e } \quad F_{A, B}(t+i \beta)=\omega\left(B \tau_{t}(A)\right) \quad t \in \mathbb{R}
$$


Chamamos de condição modular se temos uma condição KMS para $\beta=1$. Esta condição implica (usando alguns simples argumentos de análise complexa) que $\omega\left(\alpha_{t}(A)\right)=\omega(A)$, que é um fato necessário paraa caracterização de estados de equilíbrio.

Pode se mostrar que esta condição determina o estado de Gibbs unicamente sobre a álgebra $C^{*}$ dos operadores compactos. Vemos assim que, de fato, esta condição caracteriza o estado de equilíbrio nestas circunstâncias.

Utilizando-se da teoria modular de Tomita e Takesaki [8, 30], é possível conhecer e determinar de forma única o grupo uniparamétrico de ${ }^{*}$-automorfismo que torna um estado fiel e normal um estado KMS, ou seja, qual grupo uniparamétrico satisfaz a condição KMS. Para isto, enunciaremos o Teorema a seguir:

TeOrema 3.2.2 (DE TOMita e TAKesAki): Seja $\mathcal{M}$ uma álgebra de von Neumann agindo num espaço de Hilbert $\mathcal{H}$ com um vetor $\Omega$ ciclico e separante (ou seja $\mathcal{M} \Omega$ é denso em $\mathcal{H}$ e se $A \Omega=0 \Rightarrow A=0)$. Então definindo os operadores

$$
\begin{gathered}
S_{0}: \mathcal{M} \Omega(\subset \mathcal{H}) \rightarrow \mathcal{H} \text { tal que } S_{0} A \Omega \rightarrow A^{*} \Omega \quad A \in \mathcal{M} \\
F_{0}: \mathcal{M}^{\prime} \Omega(\subset \mathcal{H}) \rightarrow \mathcal{H} \text { tal que } F_{0} A^{\prime} \Omega \rightarrow A^{\prime *} \Omega \quad A^{\prime} \in \mathcal{M}^{\prime},
\end{gathered}
$$

temos que ambos são fecháveis (com fechos $S=\overline{S_{0}}$ e $F=\overline{F_{0}}$ ) e satisfazem $\left(F^{*}=S\right.$ ). Além disto, fazendo a decomposição polar destes operadores obtemos:

$$
S=J \Delta^{\frac{1}{2}}, \quad \Delta=F S, \quad \Delta^{-1}=S F, \quad J^{2}=I, \quad J \Delta^{i t}=\Delta^{i t} J, \quad J \Omega=\Delta \Omega=\Omega
$$

$$
J \mathcal{M} J=\mathcal{M}^{\prime}, \quad \Delta^{i t} \mathcal{M} \Delta^{-i t}=\mathcal{M}, t \in \mathcal{R}
$$

em que J é um operador anti-unitário e $\Delta$ é um operador positivo. Chamaremo-los de conjugação modular e operador modular respectivamente.

Utilizando-se do Teorema acima podemos formar um grupo uniparamétrico na álgebra de von Neumann, $\mathcal{M}$, em questão. O grupo uniparamétrico, chamado de grupo modular, será dado por $\sigma_{t}(A)=\Delta^{i t} A \Delta^{-i t}$. Obtemos assim o importante resultado:

TEOREMA 3.2.3: Seja $\left\{\sigma_{t}, t \in \mathbb{R}\right\}$ o grupo uniparamétrico de automorfismo modular de uma álgebra de von Neumann e $\Omega$ o vetor cíclico e separante usado para construí-lo. Então $\sigma_{t}$ satisfaz a condição modular em relação ao estado $\omega(A)=(\Omega, A \Omega)$, com $\|\Omega\|=1$. 
Este resultado se torna ainda mais interessante se acrescentarmos certos fatos. Suponhamos que tenhamos uma álgebra de von Neumann, $\mathcal{M}$ e seja dado um estado normal e fiel. Então, prova-se que a construção GNS é tal que o vetor $\Omega$ associado ao estado $\omega$ é cíclico e separante além da representação GNS, $\varphi$, ser fiel. Desta forma podemos aplicar o Teorema de Tomita e obter um grupo de automorfismos em $\mathcal{M}$ da seguinte forma:

$$
\sigma_{t}(A)=\varphi^{-1}\left(\Delta^{i t} \varphi(A) \Delta^{-i t}\right)
$$

A importância deste grupo é grande devido à seguinte proposição:

Proposição 3.2.4: (E.G. [30]) Se w é um estado normal e fiel de uma álgebra de von Neumann, $\mathcal{M}$. Então existe um único grupo de ${ }^{*}$-automorfismos de $\mathcal{M}, \sigma_{t}$, que satisfaz a condição modular em relação à $\omega$ e ele é dado por

$$
\sigma_{t}(A)=\varphi^{-1}\left(\Delta^{i t} \varphi(A) \Delta^{-i t}\right)
$$

A relevância deste fato é que ele nos permite caracterizar um estado de equilíbrio. Vemos assim que um estado de equilíbrio, com temperatura inversa $\beta$, pode ser caracterizado como um estado fiel e normal $\omega$ cujo grupo de automorfismo modular é o grupo de translação temporal $\operatorname{com} t=-\beta \tau$.

Por fim, gostaríamos de terminar esta seção mostrando outra caracterização de estados KMS. Para tal seguiremos [15] e [30]. Inicialmente enunciaremos o Teorema "Edge of the Wedge" para uma variável complexa que será fundamental na discussão a seguir: (o enunciado foi extraído de [18])

TeOrema 3.2.5 (Edge of the WedGe): Seja $F_{1}$ uma função holomorfa num conjunto aberto $D_{1}$ na metade superior do plano complexo $\{z \in \mathbb{C} ; \operatorname{Im} z>0\}$ com um intervalo aberto $a<x<b$ como parte de sua fronteira. Seja $F_{2}$ uma função holomorfa num conjunto aberto $D_{2}$ na metade inferior do plano complexo com $a<x<b$ também fazendo parte de sua fronteira. Suponha que existam para $a<x<b$

$$
F_{1}(x)=\lim _{y \rightarrow 0^{+}} F_{1}(x+i y) \text { e } F_{1}(x)=\lim _{y \rightarrow 0^{-}} F_{2}(x+i y)=F_{2}(x)
$$

Se supormos também que $F_{1}(x)$ e $F_{2}(x)$ são contínuas e satisfazem $F_{1}(x)=F_{2}(x)$ para todo $a<x<b$. Então $F_{1}$ e $F_{2}$ são, na verdade, holomorfas em $a<x<b$ e são a mesma função holomorfa.

Também de grande utilidade para expressar a unicidade de certas expressões vamos enunciar 
o Teorema a seguir (extraído de [8]):

TeOrema 3.2.6: Seja $\mathcal{O} \subset \mathbb{C}$ um conjunto aberto e conexo tal que $\mathcal{V} \equiv \mathcal{O} \cap \mathbb{R} \neq \emptyset$ e definamos:

$$
\mathcal{D}=\{z ; z=x+i y \in \mathbb{C}, y>0\} \cap \mathcal{O}
$$

Seja $F$ uma função complexa que é holomorfa em $\mathcal{D}$ e contínua em $\mathcal{D} \cup \mathcal{V}$. Suponha, além disto, que $F(x)=0$ para $x \in \mathcal{V}$. Então segue que $F(z)=0$ para todo $z \in \mathcal{D}$.

Desta forma, se duas funções são holomorfas em $\mathcal{D}$ e coincidem em $\mathbb{R}$, então estas funções são iguais.

Seguiremos agora os passos de [15]. Seja $\omega$ um estado numa álgebra $C^{*}, \mathcal{B}$, que satisfaz a condição KMS. Então na representação GNS podemos construir um espaço de Hilbert $\mathcal{H}$, uma representação $\pi$ de $\mathcal{B}$ em $\mathcal{H}$, um vetor unitário $\Omega \in \mathcal{H}$ cíclico para $\pi(\mathcal{B})$, tal que $\omega(A)=$ $(\Omega, \pi(A) \Omega)$. Estes objetos são únicos a não ser por isomorfismos, tal como foi visto na seção anterior. Também existe um grupo uniparamétrico de operadores unitários $V(t)$ em $\mathcal{H}$ que deixa $\Omega$ invariante e é tal que para todo $A \in \mathcal{B}$ temos $\pi\left(\alpha_{t}(A)\right)=V(t) \pi(A) V(t)^{*}$. Para ver isto vamos seguir [13]:

Definamos o operador $V(t)$ por

$$
V(t) \pi(A) \Omega=\pi\left(\alpha_{t}(A)\right) \Omega .
$$

Para ver que este operador está bem definido basta notar que ele preserva a norma:

$$
\|V(t) \pi(A) \Omega\|^{2}=\left\|\pi\left(\alpha_{t}(A)\right) \Omega\right\|^{2}=\omega\left(\alpha_{t}\left(A^{*} A\right)\right)=\omega\left(A^{*} A\right)=\|\pi(A) \Omega\|^{2} .
$$

Usando o Teorema BLT (ver [6]) pode-se mostrar que este operador se estende a um operador unitário (lembrando que $\pi(\mathcal{B}) \Omega$ é denso em $\mathcal{H}$ ). Agora vamos ver que ele é uma representação do grupo aditivo dos reais por operadores unitários. Para isto vejamos que $U_{s} U_{t} \pi(A) \Omega=$ $U_{s} \pi\left(\alpha_{t}(A)\right) \Omega=\pi\left(\alpha_{s}\left(\alpha_{t}(A)\right)\right) \Omega=\pi\left(\alpha_{s+t}(A)\right) \Omega=U_{s+t} \pi(A) \Omega$, a relação $U_{s} U_{t}=U_{s+t}$ é então obtida utilizando a continuidade dos operadores e o fato do vetor $\Omega$ ser cíclico. Notamos agora que como $\omega\left(A \alpha_{t}(B)\right)=\left(\pi\left(A^{*}\right) \Omega, V(t) \pi(B) \Omega\right)$ é contínuo em $t$ (pela condição KMS), então $t \rightarrow V(t)$ é fracamente contínua num conjunto denso de $\mathcal{H}$ e como $V(t)$ são operadores limitados de norma $\leq 1$ concluímos que eles são fracamente contínuas em todo o espaço $\mathcal{H}$. Isto implica em continuidade forte pois (ver [6]):

$$
\|V(t) \varphi-\varphi\|^{2}=(V(t) \varphi-\varphi, V(t) \varphi-\varphi)=(V(t) \varphi, V(t) \varphi)-(\varphi, V(t) \varphi)-(V(t) \varphi, \varphi)+(\varphi, \varphi)
$$




$$
=2\|\varphi\|^{2}-(\varphi, V(t) \varphi)-(V(t) \varphi, \varphi) \rightarrow 0 \text { quando t vai a zero. }
$$

Assim quando $t$ vai à $t_{0}$ temos $V(t) \varphi=V\left(t_{0}\right) V\left(t-t_{0}\right) \varphi \rightarrow V\left(t_{0}\right) \varphi$, pois $t-t_{0} \rightarrow 0$. Pelo Teorema de Stone [6] podemos escrever $V(t)=e^{i t H}$ em que $H$ é um operador autoadjunto. Com estas definições, mostraremos agora uma segunda forma de caracterizar um estado KMS na representação GNS. Inicalmente iremos provar uma proposição extraída de [15] que completamos alguns detalhes.

ProposiçÃo 3.2 .7 [15]: Dado um estado KMS com as definições acima, então para todos $A \in \mathcal{B}$, temos $\pi(A) \Omega \in \mathcal{D}\left(e^{-\frac{\beta}{2} H}\right)$ e, portanto, $e^{i z H} \pi(A) \Omega$ é analitico na norma na faixa aberta $I_{\beta}=\{z \in \mathbb{C} ; 0<\operatorname{Im} z<\beta / 2\}$ e continuo na norma na faixa fechada $\bar{I}_{\beta}$.

Prova: Para a prova do Teorema usaremos o Teorema espectral.

Seja $f(t)=\left(\pi(A) \Omega, e^{i t H} \pi(A) \Omega\right)=\int_{]-\infty, \infty[} e^{i t \lambda} d \mu(\lambda)$ em que estamos integrando sobre a medida $d \mu(\lambda)=(\pi(A) \Omega, d E(\lambda) \pi(A) \Omega)$ que é uma medida de probabilidade regular de Borel. Sabemos então que $f(t)=\left(\Omega, \pi\left(A^{*}\right) e^{i t H} \pi(A) e^{-i t H} \Omega\right)=\left(\Omega, \pi\left(A^{*}\right) \pi\left(\alpha_{t}(A)\right) \Omega\right)=\omega\left(A^{*} \alpha_{t}(A)\right)$. Logo, como $\omega$ é um estado KMS, sabemos que $f(t)$ admite uma extensão analítica $F(z)$ em $I_{\beta}$ e contínua em $\bar{I}_{\beta}$. Vamos agora separar $f(t)$ em duas funções $f(t)=f_{-}(t)+f_{+}(t)$ em que $f_{+}(t)=\int_{[0, \infty[} e^{i t \lambda} d \mu(\lambda)$ e $f_{-}(t)=\int_{]-\infty, 0[} e^{i t \lambda} d \mu(\lambda)$. Nosso objetivo é estudar a função $f_{-}(t)$ a fim de mostrar que $\int_{]-\infty, 0[} e^{-\beta \lambda} d \mu(\lambda)<\infty$. Com isto, poderemos concluir que $\int_{\mathbb{R}} e^{-\beta \lambda} d \mu(\lambda)<\infty$ e, portanto, pelo Teorema espectral (vide por exemplo [14]), isto implica que $\pi(A) \in \mathcal{D}\left(e^{-\frac{\beta}{2} H}\right)$.

Inicialmente veremos que $f_{-}(t)$ tem uma continuação analítica para $\operatorname{Im} z<0$. Para isto, provaremos que $\frac{d}{d z} \int_{-\infty, 0[} e^{i z \lambda} d \mu(\lambda)=\int i \lambda e^{i z \lambda} d \mu(\lambda)$. Para tanto, fixemos um $z$ tal que $\operatorname{Im} z<0$ e consideremos uma seqüência $h_{n} \in \mathbb{C}$ que vai a zero, tal que $\operatorname{Im} h_{n}<-\operatorname{Im} z / 2$. Assim basta provar que

$$
\int_{]-\infty, 0[}\left(i \lambda e^{i z \lambda}-\frac{e^{i\left(z+h_{n}\right) \lambda}-e^{i z \lambda}}{h_{n}}\right) d \mu(\lambda) \rightarrow 0 .
$$

Notemos que a função no interior da integral converge pontualmente. Para terminar a prova basta mostrar que a função é dominada. Para tanto usaremos que para todo $n$, existe algum $h_{2}$ (que independe de $n$ ) que satisfaz $\operatorname{Im} h_{2} \leq-\operatorname{Im} z / 2$ e é tal que $\left|\frac{e^{i h_{n} \lambda}-1}{h_{n}}\right| \leq\left|i h_{2} e^{i h_{2} \lambda}\right|=$ $\left|h_{2}\right|\left|e^{i h_{2} \lambda}\right|$. Assim

$$
\begin{gathered}
\int_{]-\infty, 0[}\left|i \lambda e^{i z \lambda}-\frac{e^{i\left(z+h_{n}\right) \lambda}-e^{i z \lambda}}{h_{n}}\right| d \mu(\lambda) \leq \int_{]-\infty, 0[}\left|e^{i z \lambda}\left(i \lambda-\frac{e^{i h_{n} \lambda}-1}{h_{n}}\right)\right| d \mu(\lambda) \\
\leq \int_{]-\infty, 0[} e^{-\lambda \operatorname{Im} z}\left|i \lambda-\frac{e^{i h_{n} \lambda}-1}{h_{n}}\right| d \mu(\lambda) \leq \int_{]-\infty, 0[}\left(|\lambda| e^{-\lambda \operatorname{Im} z}+\left|\frac{e^{i h_{n} z}-1}{h_{n}} e^{-\lambda \operatorname{Im} z}\right|\right) d \mu(\lambda)
\end{gathered}
$$




$$
\leq \int_{]-\infty, 0[}|\lambda| e^{-\lambda I m z} d \mu(\lambda)+\int_{]-\infty, 0[}\left|h_{2}\right| e^{-\lambda\left(\operatorname{Im} h_{2}+\operatorname{Im} z\right)} d \mu(\lambda)<\infty .
$$

Acima usamos que as funções são limitadas e a medida é finita. De forma absolutamente análoga se mostra que $f_{+}(t)$ tem continuação analítica para $\operatorname{Im} z>0$.

Agora consideremos a função $f_{-}(t)=f(t)-f_{+}(t)$. Sabemos que $f(t)$ tem continuação analítica para $I_{\beta}$, como $f_{+}(t)$ também o tem, concluímos que $f_{-}(t)$ tem continuação analítica para $I_{\beta}$. Como também a tem para $\operatorname{Im} z<0$ e as duas continuações coincidem em $\mathbb{R}$ e são contínuas lá concluímos pelo Teorema "Edge of the Wedge" que $f_{-}(t)$ tem continuação analítica, $G(z)$, para $z$ tal que $\operatorname{Im} z \in]-\infty, \beta[$ e contínua para $z$ tal que $\operatorname{Im} z \in]-\infty, \beta] . G(z)$ será igual à continuação analítica de $f_{-}(t)$ na região $\left.\operatorname{Im} z \in\right]-\infty, 0[$. Agora calcularemos sua série de Taylor ao longo do ponto 0 . Para tanto notemos que para $y=\operatorname{Im} z<0$ temos por argumentos absolutamente análogo aos anteriores que $i^{n} \frac{d^{n}}{d z^{n}} G(i y)=\frac{d^{n}}{d y^{n}} G(i y)=\int_{-\infty, 0[}(-\lambda)^{n} e^{-\lambda y} d \mu(\lambda)$. Como $f_{-}$é holomorfa num aberto contendo 0 e todas as derivadas são contínuas na região em que ela é holomorfa, concluímos que para calcular a derivada anterior no 0 basta tomar uma seqüência $y_{n} \rightarrow 0$ tal que $y_{n}<0$ e fazer $\lim _{m \rightarrow \infty} \frac{d^{n}}{d y^{n}} G\left(i y_{m}\right)=\frac{d^{n}}{d y^{n}} G(0)$. Pelo Teorema da convergência monótona temos $\lim _{m \rightarrow \infty} \int_{]-\infty, 0[}(-\lambda)^{n} e^{-\lambda y m} d \mu(\lambda) \rightarrow \int_{]-\infty, 0[}(-\lambda)^{n} d \mu(\lambda)$. Assim como $G(z)$ é analítico para $\operatorname{Im} z<\beta$ concluímos que para $0 \leq y<\beta$ a série de Taylor converge absolutamente. Portanto como $\frac{d^{n} f}{d z^{n}}=\frac{1}{i^{n}} \frac{d f}{d y^{n}}$ concluímos

$$
\begin{gathered}
G(i y)=\sum_{n=0}^{\infty} \frac{1}{i^{n}} \frac{(i y)^{n}}{n !} \frac{d^{n} G}{d y^{n}}(0)=\sum_{n=0}^{\infty} \int_{]-\infty, 0[} \frac{(-\lambda y)^{n}}{n !} d \mu(\lambda)= \\
=\int_{]-\infty, 0[} \sum_{n=0}^{\infty} \frac{(-\lambda y)^{n}}{n !} d \mu(\lambda)=\int_{]-\infty, 0[} e^{-y \lambda} d \mu(\lambda)<\infty
\end{gathered}
$$

em que se usou o Teorema da convergência monótona (lembrando que $-\lambda y>0$ na região de integração).

Novamente pelo Teorema da convergência monótona e sabendo que $G(i \beta)<\infty$ concluímos que:

$$
G(i \beta)=\lim _{y \rightarrow \beta^{-}} G(i y)=\int_{]-\infty, 0[} e^{-\beta \lambda} d \mu(\lambda)<\infty .
$$

Assim, provamos que $\int_{\mathbb{R}} e^{-\beta \lambda} d \mu(\lambda)<\infty$ e, assim, o resultado sai do Teorema espectral, como se queria. Também nota-se que para $z \in I_{\beta}$ temos $\int_{\mathbb{R}} e^{i z \lambda} d \mu(\lambda)<\infty$ pois $\left|\int_{\mathbb{R}} e^{i z \lambda} d \mu(\lambda)\right|$ $<\int_{\mathbb{R}} e^{-\lambda y} d \mu(\lambda)<\infty$. Assim, de forma análoga à anterior, e usando o Teorema da convergência dominada, prova-se que $\int_{\mathbb{R}} e^{i z \lambda} d \mu(\lambda)$ é analítica em $I_{\beta}$ e contínua em seu fecho. Como esta função coincide em $\mathbb{R}$ com $f(t)$, então, pelos Teoremas enunciados anteriormente de análise complexa, concluímos que a extensão analítica de $f(t)$ em $I_{\beta}$ é igual à $\int_{\mathbb{R}} e^{i z \lambda} d \mu(\lambda)$.

Agora, para mostrar que a função $e^{i z H} \pi(A) \Omega$ é analítica na norma em $I_{\beta}$, basta mostrar a analiticidade fraca, ou seja, que $\left(\psi, e^{i z H} \pi(A) \Omega\right.$ ) é analítica para qualquer que seja $\psi$ (pois 
analiticidade fraca equivale a analiticidade forte como é provado em [6]). Para isto, vejamos que se $\psi=\pi(B) \Omega$ em que $B \in \mathcal{B}$ temos que

$$
\begin{gathered}
\left(\pi(B) \Omega, e^{i z H} \pi(A) \Omega\right)=\frac{1}{4}\left\{\left(\pi(A+B) \Omega, e^{i z H} \pi(A+B) \Omega\right)-\left(\pi(A-B) \Omega, e^{i z H} \pi(A-B) \Omega\right)\right. \\
\left.+i\left(\pi(A+i B) \Omega, e^{i z H} \pi(A+i B) \Omega\right)-i\left(\pi(A-i B) \Omega, e^{i z H} \pi(A-i B) \Omega\right)\right\} .
\end{gathered}
$$

Como já foi visto cada uma das expressões podem ser escritas como $\int e^{i z \lambda} d \mu(\lambda)$ pelo Teorema espectral, e portanto, são analíticas em $I_{\beta}$ como já foi provado anteriormente. Se supormos $\psi$ arbitrário, então existe uma seqüência $\pi\left(B_{n}\right) \Omega \rightarrow \psi$ e assim vemos que $\left(\pi\left(B_{n}\right) \Omega, e^{i z H} \pi(A) \Omega\right)$ vai a $\left(\psi, e^{i z H} \pi(A) \Omega\right)$ uniformemente e, portanto, $\left(\psi, e^{i z H} \pi(A) \Omega\right)$ é analítico, por ser limite uniforme de funções analíticas. Para ver que o limite é uniforme notemos que:

$$
\begin{aligned}
& \left|\left(\pi\left(B_{n}\right) \Omega, e^{i z H} \pi(A) \Omega\right)-\left(\psi, e^{i z H} \pi(A) \Omega\right)\right|=\left|\left(\pi\left(B_{n}\right) \Omega-\psi, e^{i z H} \pi(A) \Omega\right)\right| \\
& \leq\left\|\pi\left(B_{n}\right) \Omega-\psi\right\|\left\|e^{i z H} \pi(A) \Omega\right\| \leq\left\|\pi\left(B_{n}\right) \Omega-\psi\right\|\left\|\left(I+e^{-\beta H}\right) \pi(A) \Omega\right\|
\end{aligned}
$$

Sendo que a última expressão vai a zero independente de $z$. Note também que usamos o Teorema espectral na última expressão da seguinte forma:

$$
\begin{aligned}
& \left\|e^{i z H} \pi(A) \Omega\right\|=\left(\int\left|e^{i z t}\right|^{2} d \mu(t)\right)^{\frac{1}{2}}=\left(\int e^{-2 y t} d \mu(t)\right)^{\frac{1}{2}} \leq\left[\int\left(1+e^{-\beta t}\right) d \mu(t)\right]^{\frac{1}{2}} \\
& \leq\left[\int\left(1+2 e^{-\frac{\beta}{2}}+e^{-\beta t}\right) d \mu(t)\right]^{\frac{1}{2}} \leq\left[\int\left(1+e^{-\frac{\beta}{2}}\right)^{2} d \mu(t)\right]^{\frac{1}{2}}=\left\|\left(I+e^{-\frac{\beta}{2}}\right) \pi(A) \Omega\right\| .
\end{aligned}
$$

Agora podemos provar o fato de maior importância que se queria, uma segunda caracterização dos estados KMS.

TEOREMa 3.2.8: Dado um estado $\omega$ numa álgebra $C^{*}, \mathcal{A}$, e sua representação GNS, então $e^{i t H}$ define um grupo de automorfismo que satisfaz a condição KMS se, e somente se, tivermos:

$$
\left(e^{-(\beta / 2) H} \pi(A) \Omega, e^{-(\beta / 2) H} \pi(B) \Omega\right)=\left(\pi\left(B^{*}\right) \Omega, \pi\left(A^{*}\right) \Omega\right) .
$$

para $\Omega$ seu vetor $G N S, \pi$ sua representação GNS e qualquer que seja $A$ e $B$ em $\mathcal{A}$.

Esta condição equivale, pela fórmula de polarização, a 


$$
\left\|e^{-(\beta / 2) H} \pi(A) \Omega\right\|=\left\|\pi\left(A^{*}\right) \Omega\right\|
$$

para todo $A \in \mathcal{A}$.

Prova: Para provar este Teorema faremos a seguinte observação preliminar. Sejam duas funções $f(z)$ e $g(z)$ de $\mathbb{C}$ a $\mathcal{H}$, sendo $\mathcal{H}$ um espaço de Hilbert. Suponha que ambas sejam analíticas na norma. Então a função $(f(\bar{z}), g(z))$ é analítica. A prova é simples. Basta ver que:

$$
\begin{gathered}
\frac{1}{h}[(f(\bar{z}+\bar{h}), g(z+h))-(f(\bar{z}), g(z))]= \\
=\frac{1}{h}[(f(\bar{z}+\bar{h}), g(z+h))-(f(\bar{z}+\bar{h}), g(z))+(f(\bar{z}+\bar{h}), g(z))-(f(\bar{z}), g(z))]= \\
=\left(f(\bar{z}+\bar{h}), \frac{g(z+h)-g(z)}{h}\right)+\left(\frac{f(\bar{z}+\bar{h})-f(\bar{z})}{\bar{h}}, g(z)\right) .
\end{gathered}
$$

Esta expressão, no limite em que $h$ vai a zero, converge à $\left(f(\bar{z}), \frac{d g}{d z}(z)\right)+\left(\frac{d}{d z} f(\bar{z}), g(z)\right)$. Logo o limite existe e a função é analítica.

Agora vamos provar o Teorema.

$(\Rightarrow)$ Vamos definir a função

$$
h(z)=\left(e^{-i \frac{\bar{z}}{2} H} \pi(A) \Omega, e^{i \frac{z}{2} H} \pi(B) \Omega\right) .
$$

Esta função é analítica em $\{z \in \mathbb{C} ; \operatorname{Im} z \in] 0, \beta[\}$ e contínua no fecho deste conjunto pela observação anterior, já que $e^{i \frac{z}{2} H}$ é analítico nesta região e contínuo em seu fecho. Agora notemos que $h(t)=\left(\pi(A) \Omega, e^{i t H} \pi(B) \Omega\right)=\omega\left(A^{*} \alpha_{t}(B)\right)$. Logo pelo Teorema 3.2.6, enunciado acima, de análise complexa sabemos que $h(z)=F_{A^{*}, B}(z)$. Assim pela condição KMS concluímos que $h(i \beta)=\omega\left(B \alpha_{0}\left(A^{*}\right)\right)=\omega\left(B A^{*}\right)=\left(\pi\left(B^{*}\right) \Omega, \pi\left(A^{*}\right) \Omega\right)$. Porém $h(i \beta)=\left(e^{-\beta H} \pi(A) \Omega, e^{-\beta H} \pi(B) \Omega\right)$. Obtemos assim o resultado esperado.

$(\Leftarrow)$ Se $\pi(A) \Omega \in \mathcal{D}\left(e^{-\beta H}\right)$ para qualquer $A \in \mathcal{B}$ obtemos, utilizando-se do Teorema espectral de forma análoga às demonstrações feitas nos Teoremas anteriores que $e^{i z H} \pi(A) \Omega$ é analítico em $I_{\beta}$ e contínuo em $\bar{I}_{\beta}$. Desta forma podemos definir a função analítica $h(z)$, como feito anteriormente, que é analítica em $I_{\beta}$ e contínua em $\bar{I}_{\beta}$. Assim obtemos que $h(t)=\omega\left(A^{*} \alpha_{t}(B)\right)$ e

$$
\begin{gathered}
h(t+i \beta)=\left(e^{-i \frac{t}{2} H-\frac{\beta}{2} H} \pi(A) \Omega, e^{i \frac{t}{2} H-\frac{\beta}{2} H} \pi(B) \Omega\right)=\left(e^{-i t H-\frac{\beta}{2} H} \pi(A) \Omega, e^{-\frac{\beta}{2} H} \pi(B) \Omega\right) \\
\left(e^{-\frac{\beta}{2} H} \pi\left(\alpha_{t}(A)\right) \Omega, e^{-\frac{\beta}{2} H} \pi(B) \Omega\right)=\left(\pi\left(B^{*}\right) \Omega, \pi\left(\alpha_{t}\left(A^{*}\right)\right) \Omega\right)=\omega\left(B \alpha_{t}(A)\right) .
\end{gathered}
$$

Logo o estado $\omega$ satisfaz a condição KMS sendo que $F_{A^{*}, B}(z)=h(z)$ 
É interessante, por fim, entender o resultado acima sob o ponto de vista do Teorema de Tomita-Takesaki. Se $\omega$ é um estado normal e fiel que satisfaz a condição KMS, então na representação GNS temos que $V(t)=e^{i t H}=\Delta^{i \frac{t}{\beta}}$ em que $\Delta$ é o operador modular, logo $e^{H}=\Delta^{\frac{1}{\beta}}$. Assim obtemos a relação acima da seguinte forma:

$$
\begin{gathered}
\left(e^{-(\beta / 2) H} \pi(A) \Omega, e^{-(\beta / 2) H} \pi(B) \Omega\right)=\left(\Delta^{\frac{1}{2}} \pi(A) \Omega, \Delta^{\frac{1}{2}} \pi(B) \Omega\right)= \\
=\left(J \Delta^{\frac{1}{2}} \pi(B) \Omega, J \Delta^{\frac{1}{2}} \pi(A) \Omega\right)=\left(\pi\left(B^{*}\right) \Omega, \pi\left(A^{*}\right) \Omega\right) .
\end{gathered}
$$

Na segunda igualdade, usamos que $J$ é um operador anti-unitário e, na terceira igualdade, que $S=J \Delta^{\frac{1}{2}}$. Assim, a segunda caracterização pode ser vista como conseqüência do Teorema de Tomita-Takesaki (Porém ele não é necessário para tanto).

Após estudarmos um pouco sobre os estados de equilíbrio, é interessante conhecermos alguns fundamentos da teoria quântica de campos. Para tanto vamos enunciar e mostrar algumas características dos axiomas de Wightman.

\subsection{Axiomas de Wightman}

Os axiomas de Wightman são um esquema axiomático surgido na década de 50 numa tentativa de formular a teoria de campos de forma matematicamente mais precisa. Ele só foi publicado, entretanto, em 1964 após a Haag e Ruelle fazerem sua teoria de espalhamento baseada neste esquema. Os axiomas de Wightman além de fornecerem definições matemáticas precisas, também fornecem resultados qualitativos corretos, entre os quais talvez o mais famoso é a conexão spin-estatística. Esta conexão explica de certa forma porque campos de spin inteiro comutam e de spin semi-inteiro anti-comutam.

Os axiomas de Wightman permitem justificar uma abordagem Euclidiana da teoria de campos como será visto mais adiante. Eles permitem assim estudar conexões profundas entre teoria de campos e mecânica estatística, (ver, por exemplo, a referência [29]).

No trabalho desenvolvido, concentraremos nossa atenção nos campos escalares de um ponto de vista axiomático. Para tanto, restringiremo-nos ao estudo de um campo quântico escalar que satisfaça os axiomas de Wightman. A seguir, vamos enunciá-los:

Axiomas de Wightman para um campo escalar [18]:

(W1) Os estados na teoria são descritos por vetores (a não ser por um fator de módulo 1) num espaço de Hilbert separável $\mathcal{H}$. A lei de transformação relativística dos estados é dada por uma representação unitária fortemente contínua do grupo restrito de Poincaré $\mathcal{P}_{+}^{\uparrow}$ :

$$
\{a, A\} \rightarrow U(a, A)
$$

em que $A \in \mathcal{P}_{+}^{\uparrow}$ e $a \in \mathbb{R}^{4}$. Como o conjunto dos operadores $\left\{U(a, 1) ; a \in \mathbb{R}^{4}\right\}$ é um mapa 
fortemente contínuo de $\mathbb{R}^{4}$ nos operadores unitários, satisfazendo $U(0,1)=1$ e $U(a, 1) U(b, 1)=$ $U(a+b, 1)$ temos (pelo Teorema VIII.12 da referência [6]) que $U(a, 1)=\exp \left(i P^{\mu} a_{\mu}\right)$, em que $P^{\mu}$ é um operador auto-adjunto não limitado (operador de energia, $P^{0}$, e de momento, $P^{i}$ para $i=1,2,3)$. Interpretamos $P^{\mu} P_{\mu}=m^{2}$ como o valor da massa ao quadrado para um estado de uma partícula.

(W2) A medida de projeção espectral (vide [6]) de $\mathbb{R}^{4}$ correspondente ao operador $U(a, 1)=$ $\exp \left(i P^{\mu} a_{\mu}\right)$ tem suporte no cone futuro $V_{+} \equiv\left\{x \in \mathbb{R}^{4}: t^{2}-x^{2}>0\right.$ e $\left.t>0\right\}$.

(W3) Existe um único vetor unitário $\Psi_{0}$, chamado de vácuo, que é invariante por qualquer operador $U(a, A)$, ou seja, $U(a, A) \Psi_{0}=\Psi_{0}$.

(W4) Para cada função $f \in \mathcal{S}\left(\mathbb{R}^{4}\right)$ (funções no espaço de Schwartz), existe um operador $\varphi(f)$ tal que, tanto ele como seu adjunto, estão definidos num conjunto denso $D$ que independe da função $f$. O conjunto $D$ contém o vetor de vácuo $\Psi_{0}$ e é invariante pela ação dos campos e dos operadores $U(a, A)$, ou seja:

$$
U(a, A) D \subset D, \quad \varphi(f) D \subset D, \quad \varphi(f)^{*} D \subset D .
$$

Dados os elementos $\phi$ e $\psi$ que pertencem a $D$ temos que $(\phi, \varphi(f) \psi): \mathcal{S}\left(\mathbb{R}^{4}\right) \rightarrow \mathbb{C}$ é uma distribuição temperada.

(W5) Os campos se transformam da seguinte forma:

$$
U(a, A) \varphi(f) U(a, A)^{-1}=\varphi(\{a, A\} f)
$$

sendo $\{a, A\} f(x)=f\left(A^{-1}(x-a)\right)$. Simbolicamente também podemos escrever:

$$
U(a, A) \varphi(x) U(a, A)^{-1}=\varphi(A x+a) .
$$

(W6) Seja $f$ e $g$ que pertencem à $\mathcal{S}\left(\mathbb{R}^{4}\right)$ com suportes causalmente separados (ou seja, $\left.f(x) g(y)=0(x-y)^{2} \geq 0\right)$, então temos que

$$
[\varphi(f), \varphi(g)] \phi=0
$$

para qualquer $\phi$ que pertença à $D$.

(W7) O espaço de Hilbert $\mathcal{H}$ é igual ao fecho da varredura linear dos vetores $\varphi\left(f_{1}\right) \ldots \varphi\left(f_{n}\right) \Psi_{0}$ para qualquer $n$ natural e $f_{1}, \ldots f_{n} \in \mathcal{S}\left(\mathbb{R}^{4}\right)$.

Com os campos quânticos satisfazendo estes postulados podemos formar as funções de Wightman. As funções de Wightman, $\mathcal{W}^{n}$, são definidas como as únicas distribuições em $\mathbb{R}^{4 n}$ tais que:

$$
\mathcal{W}^{n}\left(f_{1} \ldots f_{n}\right)=\left(\Psi_{0}, \varphi\left(f_{1}\right) \ldots \varphi\left(f_{n}\right) \Psi_{0}\right)
$$


Na expressão acima $f_{j} \in \mathcal{S}\left(\mathbb{R}^{4}\right)$ para qualquer $j$. A existência e unicidade destas funções é garantida pelo Teorema nuclear. Estas distribuições têm as seguintes propriedades:

$(F W 1) \mathcal{W}^{n}\left(x_{1}, \ldots, x_{n}\right)$ com $x_{j} \in \mathbb{R}^{4}$ são distribuições temperadas, ou seja, pertencem à $\mathcal{S}\left(\mathbb{R}^{4 n}\right)$.

(FW2) (Covariância) Existem distribuições $\mathbb{W}^{n-1} \in \mathcal{S}\left(\mathbb{R}^{4(n-1)}\right)$ tais que

$$
\mathcal{W}^{n}\left(x_{1}, x_{2}, \ldots, x_{n}\right)=\mathbb{W}^{n-1}\left(x_{2}-x_{1}, \ldots, x_{n}-x_{n-1}\right)=\mathbb{W}^{n-1}\left(\xi_{1}, \ldots, \xi_{n-1}\right)
$$

em que $x_{1}, \ldots, x_{n}, \xi_{1}, \ldots, \xi_{n-1} \in \mathbb{R}^{4}$, e $\xi_{j}=x_{j+1}-x_{j}$.

Pela expressão acima queremos dizer (ver [6]) que dado $f \in \mathcal{S}\left(\mathbb{R}^{4 n}\right)$

$$
\mathcal{W}^{n}\left(f\left(x_{1}, \ldots, x_{n}\right)\right)=\mathbb{W}^{n-1}\left(\int d x f\left(x, x-\xi_{1}, x-\xi_{1}-\xi_{2}, \ldots, x-\xi_{1}-\ldots-\xi_{n-1}\right)\right)
$$

(FW3) Esta distribuições são tais que $\mathbb{W}^{n-1}\left(\xi_{1}, \ldots, \xi_{n-1}\right)=0$ a não ser que todos $\xi_{i}$ pertencem ao cone de luz futuro, ou seja, supp $\mathbb{W}^{n-1} \subset \bar{V}_{+}^{n-1}$.

(FW4) (Localidade)

$$
\mathcal{W}^{n}\left(x_{1}, \ldots, x_{j}, x_{j+1}, \ldots, x_{n}\right)=\mathcal{W}^{n}\left(x_{1}, \ldots, x_{j+1}, x_{j}, \ldots, x_{n}\right)
$$

se $\left(x_{j}-x_{j+1}\right)^{2}<0$.

( $F W 5$ ) Seja uma seqüência de funções teste $\left\{f_{j}\right\}_{j \in \mathbb{N}}$, em que $f_{j} \in \mathcal{S}\left(\mathbb{R}^{4 j}\right)$ e $f_{j}=0$ a não ser para finitos $j^{\prime} s$. Então

$$
\sum_{j, k=0}^{\infty} \int \ldots \int \bar{f}_{j}\left(x_{1}, \ldots, x_{j}\right) f_{k}\left(y_{1}, \ldots, y_{k}\right) \mathcal{W}^{n}\left(x_{j}, \ldots, x_{1}, y_{1}, \ldots, y_{n}\right) d x_{1} \ldots d y_{n} \geq 0
$$

Em que a integral deve ser interpretada no sentido de distribuição.

(FW6) Propriedade de cluster

$$
\mathcal{W}^{n}\left(x_{1}, \ldots, x_{j}, x_{j+1}+\lambda a, \ldots, x_{n}+\lambda a\right) \rightarrow \mathcal{W}^{j}\left(x_{1}, \ldots, x_{j}\right) \mathcal{W}^{n-j}\left(x_{j+1}, \ldots, x_{n}\right)
$$

quando $\lambda \rightarrow \infty$ para a um vetor tipo espaço. A convergência é no sentido de distribuição e $\mathcal{W}^{j} \mathcal{W}^{n-j}$ deve ser interpretado como a distribuição $\mathcal{W}^{j} \otimes \mathcal{W}^{n-j}$.

Inversamente, dadas distribuições $\left\{\mathcal{W}^{n}\right\}_{n \in \mathbb{N}}$ satisfazendo (W1) a (W5) podemos reconstruir campos quânticos satisfazendo os axiomas de Wightman [18]. Para o estudo dos estados de equilíbrio estaremos interessados nas propriedades analíticas das funções de Wightman. Basicamente usaremos o seguinte Teorema:

TEOREMA 3.3.1 [18] : Existem funções holomorfas $\mathcal{W}^{n}$ e $\mathbb{W}^{n-1}$ tais que 


$$
\mathcal{W}^{n}\left(z_{1}, \ldots, z_{n}\right)=\mathbb{W}^{n-1}\left(z_{1}-z_{2}, \ldots, z_{n-1}-z_{n}\right)
$$

para $z_{1}, \ldots, z_{n} \in \mathbb{C}^{4}$.

O dominio de $\mathcal{W}^{n}$ é tal que inclui os pontos $\left(i t_{1}, \overrightarrow{x_{1}}\right), \ldots,\left(i t_{n}, \overrightarrow{x_{n}}\right)$ quando eles são dois a dois distintos.

Partindo deste Teorema podemos definir as chamadas funções de Schwinger.

DEFiniçÃo 3.3.2: Definimos a função de Schwinger por

$$
S^{n}\left(y_{1}, \ldots, y_{n}\right)=\mathcal{W}^{n}\left(z_{1}, \ldots, z_{n}\right),
$$

em que $y_{j}=\left(t_{j}, \overrightarrow{x_{j}}\right)$ e $z_{j}=\left(i t_{j}, \overrightarrow{x_{j}}\right)$ para $y_{i} \neq y_{j}$ quando $i \neq j$.

Estas funções têm as seguintes propriedades (chamadas de axiomas de Osterwalder-Schrader [17]):

$(O S 1) S^{n} \in \mathcal{S}_{\neq}^{\prime}\left(\mathbb{R}^{4 n}\right)$, ou seja, é um funcional linear contínuo no espaço $\mathcal{S}_{\neq}\left(\mathbb{R}^{4 n}\right)=\{f \in$ $\mathcal{S}\left(\mathbb{R}^{4 n}\right) ; f^{(k)}=0 \forall k$ em pontos de $\mathbb{R}^{4}$ coincidentes $\}$. Assim em $S_{\neq} f\left(x_{1}, \ldots, x_{n}\right)$ e todas as suas derivadas se anulam se $x_{i}=x_{j}$ para $i \neq j$ em que $x_{i} \in \mathbb{R}^{4}$. Neste espaço estamos considerando a topologia induzida pelo espaço $\mathcal{S}\left(\mathbb{R}^{4 n}\right)$. Estas distribuições devem satisfazer:

$$
\overline{S\left(y_{1}, \ldots y_{n}\right)}=S\left(r y_{n}, \ldots, r y_{1}\right)
$$

em que $r y_{j}=r(t, x, y, z)=(-t, x, y, z)$, para $y_{j}=(t, x, y, z)$ e $j$ de 1 a $n$. E

$$
\left|\int d y_{1} \ldots d y_{n} S\left(y_{1}, \ldots, y_{n}\right) h\left(y_{2}-y_{1}, \ldots, y_{n}-y_{n-1}\right)\right| \leq\|\check{h}\|_{\mathcal{S}\left(\mathbb{R}^{4(n-1)}\right)}
$$

sendo que $\check{f}=\int e^{-p y} f(p) d p$.

(OS2) $S^{n}\left(y_{1}, \ldots, y_{n}\right)=S^{n}\left(R y_{1}, \ldots, R y_{n}\right)$ em que $R \in S O(4)$.

(OS3) Seja uma seqüência de funções teste $\left\{f_{j}\right\}_{j \in \mathbb{N}}$, cujas funções pertencem ao conjunto $\left\{\mathcal{S}_{+}\left(\mathbb{R}^{4 j}\right)=\left\{f_{j}\left(i t_{1}, \vec{x}_{1}, \ldots, i t_{j}, \vec{x}_{j}\right) ; f_{j}=0\right.\right.$ a não ser que $\left.0<t_{1}<\ldots<t_{n}\right\}$ e $f_{j}=0$ a não ser para finitos $j^{\prime} s$. Então

$$
\sum_{j, k=0}^{\infty} \int \ldots \int \bar{f}_{j}\left(x_{1}, \ldots, x_{j}\right) f_{k}\left(y_{1}, \ldots, y_{k}\right) S^{n}\left(x_{j}, \ldots, x_{1}, y_{1}, \ldots, y_{n}\right) d x_{1} \ldots d y_{n} \geq 0
$$

em que a integral deve ser interpretada no sentido de distribuição.

$\left(O S_{4}\right)$ Seja $\pi:\{1, \ldots, n\} \rightarrow\{1, \ldots, n\}$ uma permutação. Então $S\left(y_{1}, \ldots, y_{n}\right)=S\left(y_{\pi(1)}, \ldots, y_{\pi(n)}\right)$ para qualquer que seja esta permutação.

(OS5) A propriedade do cluster é agora: 


$$
\lim _{\lambda \rightarrow \infty} S^{n+m}\left(r y_{n}, \ldots, r y_{1}, y_{1}^{\prime}+\lambda a, \ldots y_{n}^{\prime}+\lambda a\right)=S^{n}\left(r y_{n}, \ldots, r y_{1}\right) S^{m}\left(y_{1}^{\prime}, \ldots, y_{m}^{\prime}\right)
$$

Da mesma forma, dadas funções $S^{n}$ que satisfaçam estas propriedades, podemos construir uma única teoria de campo, obtendo distribuições de Wightman que satisfaçam (W1) até (W6). Assim, as funções de Schwinger caracterizam totalmente uma teoria quântica de campos. As funções de Schwinger serão essenciais a nós.

No nosso trabalho, ao estudarmos o efeito Unruh do ponto de vista Euclidiano, no entanto, partiremos de funções de Schwinger que satisfaçam uma propriedade extra dada por:

(OS2') $S^{n}\left(y_{1}, \ldots, y_{n}\right)=S^{n}\left(R y_{1}, \ldots, R y_{n}\right)$ em que $R \in O(4)$.

Ou seja, substituímos o grupo $S O(4)$ por um grupo maior $O(4)$. Esta propriedade não é satisfeita por todos os campos que satisfaçam os axiomas de Wightman, no entanto é assumida em textos clássicos tais como [5] e satisfeita por vários campos de interesse.

Para seguirmos nosso estudo, usaremos o procedimento adotado por Fröhlich e Birke [12] e vamos parametrizar as coordenadas de $\mathbb{R}^{4},(t, x, y, z)$ por $(\theta, r, y, z)$ da seguinte forma:

$$
t=r \sin \theta, \quad x=r \cos \theta
$$

Definamos agora o espaço $\mathbb{S}=\left\{f(r, y, z) \in \mathcal{S}\left(\mathbb{R}^{3}\right) ;\right.$ supp $f \subset\left[0, \infty\left[\times \mathbb{R}^{2}\right\}\right.$ e seja $a_{j} \in \mathbb{S}$ para $j=1, \ldots, n$ em que $n \in \mathbb{N}$. Vamos agora usar as seguintes funções:

$$
\begin{gathered}
\phi_{2 \pi}\left(a_{1}, \theta_{1}, \ldots, a_{n}, \theta_{n}\right):=\int S^{n}\left(t^{1}\left(\theta_{1}, r_{1}\right), x^{1}\left(\theta_{1}, r_{1}\right), y^{1}, z^{1}, \ldots, t^{n}\left(\theta_{1}, r_{1}\right), x^{n}\left(\theta_{1}, r_{1}\right), y^{n}, z^{n}\right) \\
\times \prod_{j=1}^{n} a_{j}\left(r_{j}, y_{j}, z_{j}\right) d r_{j} d y_{j} d z_{j} .
\end{gathered}
$$

Em que $\left(\theta_{1}, \ldots, \theta_{n}\right) \in \mathbb{T}_{<}=\left\{\left(\theta_{1}, \ldots, \theta_{n}\right) \in[0,2 \pi]^{n} ; \theta_{i} \neq \theta_{j}\right.$ se $\left.i \neq j\right\}$.

Estas funções satisfazem propriedades que se mostrarão cruciais ao desenvolvimento do trabalho conforme [12]:

(P1) As funções $\phi_{2 \pi}\left(a_{1}, \theta_{1}, \ldots, a_{n}, \theta_{n}\right)$ estão definidas para quaisquer $\left(a_{1}, \ldots, a_{n}\right) \in \mathcal{S}^{\times n} \mathrm{e}$ $\left(\theta_{1}, \ldots, \theta_{n}\right) \in \mathbb{T}_{<}$. É contínua conjuntamente em $\mathcal{S}^{\times n}$ na topologia produto e contínua em $\left(\theta_{1}, \ldots, \theta_{n}\right) \in \mathbb{T}_{<}=\left\{\left(\theta_{1}, \ldots, \theta_{n}\right) \in[0,2 \pi]^{n} ; \theta_{i} \neq \theta_{j}\right.$ se $\left.i \neq j\right\}$.

(P2) $\phi_{2 \pi}\left(a_{1}, \theta_{1}, \ldots, a_{n}, \theta_{n}\right)=\phi_{2 \pi}\left(a_{1}, \theta_{1}+\theta, \ldots, a_{n}, \theta_{n}+\theta\right)$.

Prova: Basta notar que a transformação $(\theta, r, y, z) \rightarrow(\theta+\psi, r, y, z)$ corresponde à transformação $(t, x, y, z)=(r \sin \theta, r \cos \theta, y, z) \rightarrow(r \sin (\theta+\psi), r \cos (\theta+\psi), y, z) \rightarrow(t \cos \psi+$ $x \sin \psi,-t \sin \psi+r \cos \psi, y, z)$. Logo a matriz de transformação de coordenadas é: 


$$
\left(\begin{array}{cccc}
\cos \psi & \sin \psi & 0 & 0 \\
-\sin \psi & \cos \psi & 0 & 0 \\
0 & 0 & 1 & 0 \\
0 & 0 & 0 & 1
\end{array}\right)
$$

Que pertence a $S O(4)$. Logo o resultado é conseqüência de (OS2).

(P3) Vale a identidade KMS

$$
\phi_{2 \pi}\left(a_{1}, \theta_{1}, \ldots, a_{n}, \theta_{n}\right)=\phi_{2 \pi}\left(a_{j+1}, \theta_{j+1}, \ldots, a_{n}, \theta_{n}, a_{1}, \theta_{1}+2 \pi, \ldots, a_{j}, \theta_{j}+2 \pi\right) .
$$

Prova: Basta notar que podemos permutar $\left(a_{j}, \theta_{j}\right)$ em $\phi_{2 \pi}$ e que as funções $\phi_{2 \pi}$ são $2 \pi$ periódicas como função de $\theta$.

$(P 4)$ Seja dado um conjunto de finitas seqüências do tipo $\left(a_{1}^{j}, \theta_{1}, \ldots, a_{n_{j}}^{j}, \theta_{n_{j}}^{j}\right)$ e $\left(\theta_{1}^{j}, \ldots, \theta_{n_{j}}^{j}\right) \in$ $[0, \pi]^{n_{j}}$ e as variáveis $\theta$ são duas a duas distintas. Então a matriz abaixo é positiva:

$$
\Pi_{i j}=\phi_{2 \pi}\left(a_{1}^{i}, \theta_{1}^{i}, \ldots, a_{n_{i}}^{i}, \theta_{n_{i}}^{i}, a_{n_{j}}^{*}, 2 \pi-\theta_{n_{j}}^{j}, \ldots, a_{1}^{* j}, 2 \pi-\theta_{1}^{j}\right) .
$$

Prova: Isto é uma conseqüência da propriedade (OS3) dos axiomas de Osterwalder-Schrader e a observação de que, para $\theta$ entre 0 e $\pi$, temos que a reversão temporal pode ser dada por $2 \pi-\theta$, pois se $\theta \rightarrow 2 \pi-\theta$ então $\sin (\theta) \rightarrow-\sin (\theta)$ e $\cos (\theta)$ permanece igual. Notemos, no entanto, que a ordenação acima não é algo crucial já que os termos $(a, \theta)$ comutam em $\phi_{2 \pi}$.

(P5) $\phi_{2 \pi}\left(a_{1}, \theta_{1}, \ldots, a_{n}, \theta_{n}\right)=\phi_{2 \pi}\left(a_{1},-\theta_{1}, \ldots, a_{n},-\theta_{n}\right)$.

Prova: Basta notar que a transformação $(\theta, r, y, z) \rightarrow(-\theta, r, y, z)$ corresponde a transformação $(t, x, y, z)=(r \sin (\theta), r \cos (\theta), y, z) \rightarrow(r \sin (-\theta), r \cos (-\theta), y, z)=(-t, x, y, z)$. Logo a matriz de transformação de coordenadas é:

$$
\left(\begin{array}{cccc}
-1 & 0 & 0 & 0 \\
0 & 1 & 0 & 0 \\
0 & 0 & 1 & 0 \\
0 & 0 & 0 & 1
\end{array}\right)
$$

Que pertence a $O(4)$. Logo o resultado é conseqüência de $\left(O S 2^{\prime}\right)$.

Estas propriedades serão muito importantes no capítulo posterior, em especial para a seção 4.3.1. Vamos, antes disso, mostrar como o trabalho de Sewell utiliza os axiomas de Wightman e os estados KMS para obter uma nova dedução do efeito Unruh. 


\subsection{O efeito Unruh de um ponto de visto algébrico (o tra- balho de Sewell)}

Para demonstrar o efeito Unruh sob um ponto de vista algébrico, novamente consideraremos campos escalares tal como Sewell (em [27]). Para tanto, seja $\left(\mathcal{H}, \varphi, \Psi_{0}, U\right)$ um campo quântico satisfazendo os axiomas de Wightman, em que $\mathcal{H}$ é um espaço de Hilbert separável, $\varphi$ o campo da teoria, ou seja, uma função linear de $\mathcal{S}\left(\mathbb{R}^{4}\right)$ em operadores de $\mathcal{H}$ em si mesmo, $\Psi_{0}$ o estado de vácuo e $U$ o operador que representa as transformações de Poincaré em $\mathcal{P}_{+}^{\uparrow}$.

Dentro desta teoria, podemos caracterizar os estados em equilíbrio térmico de forma análoga ao já mostrado quando foram discutidos os estados KMS. Assim supondo dadas uma álgebra-* (álgebra fechada pela operação de tomar o adjunto) $\mathcal{A}$ de operadores em $\mathcal{H}$ com domínio denso comum $\mathcal{K}$, representando os observáveis da teoria, e $V(t)$ uma uma representação unitária fortemente contínua do grupo aditivo dos reais $\mathbb{R}$, tal que $V(\mathbb{R}) \mathcal{K} \subset \mathcal{K}$ e tal que $V(t) \mathcal{A} V(-t) \subset$ $\mathcal{A}$ para qualquer que seja $t \in \mathbb{R}$. Dizemos, então, que um estado $\Psi \in \mathcal{K}$, o qual é invariante sobre $V(\mathbb{R})$ e cíclico com respeito a álgebra $\mathcal{A}$, é um estado térmico a uma temperatura $T=(k \beta)^{-1}$ se ele satisfizer a condição KMS, ou seja, se

$$
\left(\exp \left(-\frac{1}{2} \beta \hbar K\right) A \Psi, \exp \left(-\frac{1}{2} \beta \hbar K\right) B \Psi\right)=\left(B^{*} \Psi, A^{*} \Psi\right) \quad \forall A, B \in \mathcal{A} .
$$

$\mathrm{Ou}$, equivalentemente, se existe uma (única) conjugação tal que

$$
J \exp \left(-\frac{1}{2} \beta \hbar K\right) A \Psi=A^{*} \Psi, \quad \forall A \in \mathcal{A} .
$$

Pois repetindo o argumento feito na seção anterior temos:

$\left(\exp \left(-\frac{1}{2} \beta \hbar K\right) A \Psi, \exp \left(-\frac{1}{2} \beta \hbar K\right) B \Psi\right)=\left(J \exp \left(-\frac{1}{2} \beta \hbar K\right) B \Psi, J \exp \left(-\frac{1}{2} \beta \hbar K\right) A \Psi\right)=\left(B^{*} \Psi, A^{*} \Psi\right)$

Adotaremos como álgebra $\mathcal{A}$ o conjunto dos operadores da forma $\varphi\left(f_{1}\right) \ldots \varphi\left(f_{n}\right)$ em que $f_{j} \in$ $\mathcal{S}\left(\mathbb{R}^{4}\right)$ para qualquer $j$. Vamos, agora, nos restringir ao subespaço de Rindler $\mathcal{R}=\{(t, x, y, z) \in$ $\left.\mathbb{R}^{4} ; x>|t|\right\}$ do espaço de Minkowski. Como feito anteriormente, podemos parametrizar o espaço por:

$$
t=\xi \sinh T \quad x=\xi \cosh T .
$$

Como já foi visto, temos que as curvas $\operatorname{com} \xi, y, z$ constantes e $T$ variando correspondem a trajetórias com aceleração uniforme $c^{2} / \xi$, com tempo próprio dado por $c s=c T / \alpha$. O grupo uniparamétrico de isometrias que correspondem as translações temporais para um observador uniformemente acelerado é dado por:

$$
L^{+}(T)\left(\xi, T^{\prime}, y, z\right)=\left(\xi, T+T^{\prime}, y, z\right)
$$


como pode ser visto facilmente fazendo:

$$
\left(\begin{array}{cccc}
\cosh (T) & \sinh (T) & 0 & 0 \\
\sinh (T) & \cosh (T) & 0 & 0 \\
0 & 0 & 1 & 0 \\
0 & 0 & 0 & 1
\end{array}\right)\left(\begin{array}{c}
\xi \sinh T^{\prime} \\
\xi \cosh T^{\prime} \\
y \\
z
\end{array}\right)=\left(\begin{array}{c}
\xi \sinh \left(T+T^{\prime}\right) \\
\xi \cosh \left(T+T^{\prime}\right) \\
y \\
z
\end{array}\right)
$$

Agora basta enunciarmos dois Teoremas a fim de obter o resultado esperado.

TEOREMA DE REEH-SChliEder 3.4.1 [18]: Seja $\Lambda$ um subconjunto aberto arbitrário de $M=\mathbb{R}^{4}$ e seja $\mathcal{A}(\Lambda)$ uma sub-álgebra de $\mathcal{A}$ dada pelos polinômios em $P(\Lambda)=\left\{\varphi(f) ; f \in \mathcal{S}\left(\mathbb{R}^{4}\right)\right.$ tal que supp $(f) \subset \Lambda\}$. Então o conjunto $\mathcal{A}(\Lambda) \Psi_{0}$ é denso em $\mathcal{H}$. Além disto, se o conjunto dos pontos de $\mathbb{R}^{4}$ que são de tipo espaço em relação a todo o ponto em $\Lambda$ não é vazio, então $\Psi_{0}$ é também separante para $P(\Lambda)$.

Teorema de Bisognano-Wichmann 3.4 .2 [9, 10]: Seja $i K$ o gerador infinitesimal de $\hat{L}(\mathbb{R}):=U(L(\mathbb{R}))$. Então:

$$
J_{0} \hat{\rho} e^{-\pi K} A \Psi_{0}=A^{*} \Psi_{0}, \quad \forall A \in \mathcal{A}(\mathcal{R}),
$$

em que $J_{0}$ é a única conjugação de $\mathcal{H}$, cuja existência é garantida pelo Teorema PCT [24], tal que

$$
J_{0} \varphi\left(f_{1}\right) \ldots \varphi\left(f_{k}\right) \Psi_{0}=\varphi\left(f_{k}^{\dagger}\right) \ldots \varphi\left(f_{1}^{\dagger}\right) \Psi_{0} \quad \forall f_{1}, \ldots, f_{k} \in \mathcal{S}\left(\mathbb{R}^{4}\right)
$$

onde definimos

$$
f^{\dagger}(x):=\bar{f}(-x)
$$

e $\hat{\rho}=U(\rho)$ para $\rho$ a inversão parcial $y \rightarrow-y$ e $z \rightarrow-z$

A partir destes Teoremas obtemos o seguinte resultado

Proposição 3.4 .3 [27]: A restrição do estado de vácuo $\Psi_{0}$ a $\mathcal{A}(\mathcal{R})$ é um estado térmico satisfazendo a seguinte condição KMS com respeito ao grupo de translação temporal $\hat{L}^{(+)}(\mathbb{R})$ $(:=U(L(\mathbb{R})))$, que corresponde aos observadores acelerados:

$$
J \exp (-\pi K) A \Psi=A^{*} \Psi, \quad \forall A \in \mathcal{A}(\mathcal{R})
$$

em que $i K^{(+)}$é o gerador de $\hat{L}^{(+)}(\mathbb{R})$ e $J=J_{0} \hat{\rho}$, em que $J_{0}$ é o operador PCT e $\hat{\rho}$ é a representação unitária da inversão parcial $y \rightarrow-y$ e $z \rightarrow-z$.

Prova:

Para ver isto lembramos que $\Psi_{0}$ é invariante sobre $U(L(\mathbb{R}))$ devido aos axiomas de Wightman 
e que $\mathcal{A}(\mathcal{R}) \Psi_{0}$ é denso em $\mathcal{H}$ devido ao Teorema de Reeh-Schlieder. Notamos também que devido aos axiomas de Wightman $U(L(\mathbb{R})) \mathcal{K} \subset \mathcal{K}$ em que $\mathcal{K}$ é o domínio comum dos campos. Como os boosts levam a região $\mathcal{R}$ nela mesma, eles são tais que $U(L(\mathbb{R})) \mathcal{A}(\mathcal{R}) U(L(\mathbb{R})) \subset \mathcal{A}(\mathcal{R})$. Logo tudo está de acordo com a definição dada nesta seção de estado KMS. Por fim, observamos que $J \exp (-\pi K) A \Psi=A^{*} \Psi, \quad \forall A \in \mathcal{A}(\mathcal{R})$ é uma conseqüência direta do Teorema de BisognanoWichmann. Esta expressão é a mesma do estado KMS para $\frac{1}{2} \beta \hbar=\pi$. Logo $\Psi_{0}$ é de fato um estado térmico.I

Notamos acima que para o estado KMS acima é obtido a temperatura $T=\frac{\hbar}{2 \pi k}$. Como na verdade o tempo próprio é $c \tau / \alpha$ temos, reescalonando, que a temperatura é $T_{\alpha}=\frac{\hbar \alpha}{2 \pi k c}$. Obtemos, assim, a generalização algébrica para campos em espaços-tempos planos. 


\section{Capítulo 4}

\section{O efeito Unruh: ponto de vista estocástico}

Neste capítulo, esboçaremos uma nova estratégia para explicar o efeito Unruh. Para tanto, vamos utilizar de resultados mostrados no capítulo anterior sobre a formulação axiomática da teoria quântica dos campos. Também precisaremos mostrar resultados adicionais acerca de certas relações entre análise funcional e probabilidade.

Procuraremos, na próxima subseção, listar as principais definições sobre processos estocásticos a fim de dar sentido ao que será apresentado na explicação do trabalho de Klein, Landau, Gérard e Jäkel [15, 7]. Apresentaremos resultados sobre equivalência de processos estocásticos, assim como relações entre esperança condicional num espaço de probabilidade e operadores definidos nele, entre outros resultados relevantes. Com estes resultados em mão, acreditamos que tenhamos os pré-requisitos necessários para descrevermos o trabalho de Klein e Landau [15] e, enfim, o trabalho de Christian Gérard e Christian Jäkel [7].

Mostraremos como os dois trabalhos se relacionam, em particular, como os processos estocásticos de [15] implicam nos espaços de trajetória generalizados de [7]. Por fim mostramos qual seria um possível procedimento para fazer uma demonstração alternativa do efeito Unruh.

\subsection{Relações entre probabilidade e análise funcional}

Nesta seção vamos mostrar alguns resultados estudados e que serão utilizados para a compreensão do trabalho. Basearemos principalmente em $[2,4,15,1]$. Nosso procedimento inicial será estudar aspectos básicos da teoria de processos estocásticos para, então, definir os operadores $U(t)$ e $R$ que atuarão nos espaços $L^{p}$ do espaço de medida considerado. Definiremos, então, a esperança condicional e veremos sua relação com os operadores previamente definidos. Por fim, ao longo da seção provaremos resultados que nos serão úteis em seções posteriores.

Antes disso iremos fixar alguma notação. Chamaremos de $\sigma$-álgebra $[\mathcal{A}]$ a $\sigma$-álgebra gerada pelo conjunto $\mathcal{A}$. A mesma notação será usada para anel, $\sigma$-anel, álgebra,... Denotaremos por:

$\mathbb{R}^{\infty}$ o conjunto das seqüências de números reais.

$\mathcal{B}_{n}=\sigma$-álgebra $\left[I_{1} \times \ldots \times I_{n} \times \mathbb{R} \times \mathbb{R} \times \ldots\right]$ em que $I_{1}, \ldots, I_{n}$ são intervalos, ou seja, $\mathcal{B}_{n}$ é a $\sigma$ 
-álgebra dos n-cilindros.

$\mathcal{B}_{\infty}=\sigma$-álgebra $\left[\cup_{n=1}^{\infty} \mathcal{B}_{n}\right]$, ou seja, é a $\sigma$-álgebra $\prod_{i=1}^{\infty} \mathcal{B}(\mathbb{R})$ (Produto infinito).

$n-$ cilindro $=B \times \prod_{i=n+1}^{\infty} \mathbb{R}$, em que $B \in \mathcal{B}\left(\mathbb{R}^{n}\right)=\mathcal{B}(\mathbb{R}) \otimes \ldots \otimes \mathcal{B}(\mathbb{R})$, em que há $n$ cópias de $\mathcal{B}(\mathbb{R})$.

Agora vamos dar a definição de um processo estocástico:

DEFINIÇÃO 4.1.1 [15]: Um processo estocástico, indexado por um conjunto $T$ com valores em um espaço topológico $K$, é uma família $\left\{X_{t}\right\}_{t \in T}$ de variáveis aleatórias (funções mensuráveis) em um espaço de probabilidade $(Q, \Sigma, \mu)$ com valores em $K$, em que tomamos a $\sigma$-álgebra de $K$ como sua $\sigma$-álgebra de Borel. Denota-se por $<>$ a esperança no espaço de probabilidade, ou seja, $\langle F\rangle=\int F d \mu$ para uma função integrável $F$ em $(Q, \Sigma, \mu)$.

Vamos agora estudar alguns aspectos dos processos estocásticos com um número enumerável de variáveis aleatórias. Seja $\left\{X_{n}\right\}_{n \in \mathbb{N}}$ um processo estocástico. Logo para cada $n \in \mathbb{N}$ temos que $X_{n}:(\Omega, \mathcal{F}, \mathcal{P}) \rightarrow(\mathbb{R}, \mathcal{B}(\mathbb{R}))$ é uma função mensurável. Notemos desde já que enunciaremos os Teoremas para processos estocásticos com valores em $\mathbb{R}$, porém em grande parte das provas abaixo podemos substituir $\mathbb{R}$ e $\mathcal{B}(\mathbb{R})$ por um espaço topológico $K$ qualquer e sua $\sigma$-álgebra de Borel $\mathcal{B}(K)$.

Vamos definir uma função $\left(X_{1}, \ldots, X_{n}, \ldots\right):(\Omega, \mathcal{F}, \mathcal{P}) \rightarrow\left(\mathbb{R}^{\infty}, \mathcal{B}_{\infty}\right)$ dada por $\left(X_{1}, X_{2}, \ldots\right)(\omega)=$ $\left(X_{1}(\omega), X_{2}(\omega), \ldots\right)$. Esta função é mensurável como pode ser visto por $\left(X_{1}, X_{2}, \ldots\right)^{-1}\left(I_{1} \times \ldots \times\right.$ $\left.I_{n} \times \mathbb{R} \times \mathbb{R} \times \ldots\right)=X_{1}^{-1}\left(I_{1}\right) \cap \ldots \cap X_{n}^{-1}\left(I_{n}\right) \in \mathcal{F} \mathrm{e}\left(I_{1} \times \ldots \times I_{n} \times \mathbb{R} \times \mathbb{R} \times \ldots\right)$ gera $\mathcal{B}_{\infty}$. Com estas considerações podemos fazer a seguinte definição:

DEFINIÇÃo 4.1.2: A distribuição de probabilidade do processo $\left\{X_{n}\right\}_{n \in \mathbb{N}}$ é dada por uma função $\hat{P}: \mathcal{B}_{\infty} \rightarrow \mathbb{R}$ dada por $\hat{P}(B)=\mathcal{P}\left(\left(X_{1}, X_{2}, \ldots\right)^{-1}(B)\right)$ em que $B \in \mathcal{B}_{\infty}$. Esta função é uma medida de probabilidade, pois $\hat{P}(\mathbb{R} \times \mathbb{R} \times \ldots)=\mathcal{P}(\Omega)=1, \hat{P}(\emptyset)=0$ e $\hat{P}\left(\cup_{n=1}^{\infty} A_{n}\right)=$ $\mathcal{P}\left(\cup_{n=1}^{\infty}\left(X_{1}, X_{2}, \ldots\right)^{-1}\left(A_{n}\right)\right)=\sum_{n=1}^{\infty} \mathcal{P}\left(\left(X_{1}, X_{2}, \ldots\right)^{-1}\left(A_{n}\right)\right)=\sum_{n=1}^{\infty} \hat{P}\left(A_{n}\right)$ se $A_{n}$ são conjuntos disjuntos.

DeFiniçÃo 4.1.3: Dizemos que dois processos $\left\{X_{n}\right\}$ e $\left\{X_{n}^{\prime}\right\}$ em que $X_{n}:(\Omega, \mathcal{F}, \mathcal{P}) \rightarrow$ $(\mathbb{R}, \mathcal{B}(\mathbb{R}))$ e $X_{n}^{\prime}:\left(\Omega^{\prime}, \mathcal{F}^{\prime}, \mathcal{P}^{\prime}\right) \rightarrow(\mathbb{R}, \mathcal{B}(\mathbb{R}))$ têm a mesma distribuição de probabilidade se dado $B \in \mathcal{B}_{\infty}$ temos que $\hat{P}(B)=\mathcal{P}\left(\left(X_{1}, X_{2}, \ldots\right)^{-1}(B)\right)=\hat{\mathcal{P}}^{\prime}\left(\left(X_{1}^{\prime}, X_{2}^{\prime}, \ldots\right)^{-1}(B)\right)=\hat{P}^{\prime}(B)$.

DEFINIÇÃo 4.1.4: Dado o processo $\left\{X_{n}\right\}_{n \in \mathbb{N}}$ em $(\Omega, \mathcal{F}, \mathcal{P})$ definimos a função de distribuição n-dimensional por $F\left(x_{1}, \ldots, x_{n}\right)=\mathcal{P}\left(X_{1}<x_{1}, \ldots, X_{n}<x_{n}\right)$. Usaremos a notação $F_{n}(.) \equiv F_{n}\left(x_{n}\right) \equiv F_{X_{n}}\left(x_{n}\right) \equiv F_{X_{1}, \ldots, X_{n}}\left(x_{1}, \ldots, x_{n}\right)$

Para relacionar função de distribuição com distribuição de probabilidade usaremos o seguinte lema [31]: 
LEMA 4.1 .5 [31]: Seja $\mathcal{A}$ um $\sigma$-anel e $\mu: \mathcal{A} \rightarrow[0, \infty[, \nu: \mathcal{A} \rightarrow[0, \infty[$ medidas finitas em $\mathcal{A}$. Se $\mathcal{C} \subset \mathcal{A}$ é uma classe fechada por interseç̧ão finita e $\mathcal{A}=\sigma-$ anel $[\mathcal{C}]$ e $\mu(A)=$ $\nu(A) \forall A \in \mathcal{C}$. Então $\mu=\nu$.

Para a prova deste lema usa-se o lema da classe aditiva.

Dadas estas definições podemos agora estudar como elas se relacionam.

TeOrema 4.1.6: Dois processos, $\left\{X_{n}\right\}_{n \in \mathbb{N}} e\left\{X_{n}^{\prime}\right\}_{n \in \mathbb{N}}$, têm a mesma distribuição de probabilidade se, e somente se, suas funções de distribuição são iguais.

Prova: Seja $\hat{P}$ e $\hat{P}^{\prime}$ definidas em $\mathcal{B}_{\infty}$ por $\hat{P}(B)=\mathcal{P}\left(\left(X_{1}, X_{2}, \ldots\right)^{-1}(B)\right)$ e pela expressão $\hat{P}^{\prime}(B)=\mathcal{P}^{\prime}\left(\left(X_{1}^{\prime}, X_{2}^{\prime}, \ldots\right)^{-1}(B)\right)$ para $B \in \mathcal{B}_{\infty}$. Se $\mathcal{C} \subset \mathcal{B}_{\infty}$ é a classe de conjuntos da forma $C=]-\infty, y_{1}[\times \ldots \times]-\infty, y_{n}\left[\times \mathbb{R} \times \mathbb{R} \times \ldots\right.$, então $\mathcal{C}$ é fechado por intersecções finitas e gera $\mathcal{B}_{\infty}$. Logo

$(\Rightarrow)$ Se os processos têm a mesma distribuição de probabilidade então:

$F_{X_{1}, \ldots, X_{n}}\left(y_{1}, \ldots, y_{n}\right)=\mathcal{P}\left(X_{1}^{-1}(]-\infty, y_{1}[) \cap \ldots \cap X_{n}^{-1}(]-\infty, y_{n}[)\right)=\hat{P}(]-\infty, y_{1}[\times \ldots \times]-$ $\infty, y_{n}[\times \mathbb{R} \times \mathbb{R} \times \ldots)=\hat{P}^{\prime}(]-\infty, y_{1}[\times \ldots \times]-\infty, y_{n}[\times \mathbb{R} \times \mathbb{R} \times \ldots)=\mathcal{P}^{\prime}\left(X_{1}^{\prime-1}(]-\infty, y_{1}[) \cap \ldots \cap\right.$ $\left.X_{n}^{\prime-1}(]-\infty, y_{n}[)\right)=F_{X_{1}^{\prime}, \ldots, X_{n}^{\prime}}\left(y_{1}, \ldots, y_{n}\right)$

$(\Leftarrow)$ Se suas funções de distribuição são iguais então:

$F_{X_{1}, \ldots, X_{n}}\left(y_{1}, \ldots, y_{n}\right)=F_{X_{1}^{\prime}, \ldots, X_{n}^{\prime}}\left(y_{1}, \ldots, y_{n}\right)$. Portanto $\hat{P}(]-\infty, y_{1}[\times \ldots \times]-\infty, y_{n}[\times \mathbb{R} \times \mathbb{R} \times \ldots)$ $=\hat{P}^{\prime}(]-\infty, y_{1}[\times \ldots \times]-\infty, y_{n}[\times \mathbb{R} \times \mathbb{R} \times \ldots)$. Desta forma $\hat{P}(C)=\hat{P}^{\prime}(C)$ para todo $C \in \mathcal{C}$ e, portanto, pelo lema anterior $\hat{P}=\hat{P}^{\prime}$.

DEFInição 4.1.7: Um processo $X_{1}, X_{2}, X_{3},$. é estacionário se para $\forall k \in \mathbb{N}$, o processo $X_{k+1}, X_{k+2}, X_{k+3}, \ldots$ tem a mesma distribuição de probabilidade de $X_{1}, X_{2}, X_{3}, \ldots$ Logo para qualquer $B \in \mathcal{B}_{\infty}$ temos:

$$
\mathcal{P}\left(\left(X_{1}, X_{2}, X_{3}, \ldots\right) \in B\right)=\mathcal{P}\left(\left(X_{k+1}, X_{k+2}, \ldots\right) \in B\right) B \in \mathcal{B}_{\infty}
$$

Isto equivale a $F_{X_{1}, \ldots, X_{n}}\left(x_{1}, \ldots, x_{n}\right)=F_{X_{k+1}, \ldots, X_{k+n}}\left(x_{1}, \ldots, x_{n}\right)$ para qualquer que sejam $k, n \in$ $\mathbb{N}$, logo a definição é equivalente a $P\left(X_{1}<x_{1}, \ldots, X_{n}<x_{n}\right)=P\left(X_{k+1}<x_{1}, \ldots, X_{k+n}<\right.$ $\left.x_{n}\right)$ para qualquer que seja $k, n \in \mathbb{N}$. Concluímos, então, que as definições equivalem a $\mathcal{P}\left(\left(X_{1}, \ldots, X_{n}\right)^{-1}(A)\right)=\mathcal{P}\left(\left(X_{k+1}, \ldots, X_{k+n}\right)^{-1}(A)\right)$ para qualquer que seja $k, n \in \mathbb{N}$ e $A \in \mathcal{B}\left(\mathbb{R}^{n}\right)$, utilizando-se sempre do lema 4.1.5.

Analogamente vamos fazer definições de processos estacionários e simétricos indexados por $\mathbb{R}$.

DEFINIÇÃO 4.1.8: Um processo estocástico $\left\{X_{t}\right\}_{t \in \mathbb{R}}$ é estacionário se para qualquer que seja $s \in \mathbb{R}$ e qualquer seqüência de números reais $\left\{t_{i}\right\}_{i=1}^{\infty}$ temos que o processo $X_{t_{1}+s}, X_{t_{2}+s}, \ldots$ tem 
a mesma distribuição de probabilidade de $X_{t_{1}}, X_{t_{2}}, \ldots$ Assim equivalem

- Para $\forall B \in \mathcal{B}_{\infty}$ temos $\mathcal{P}\left(\left(X_{t_{1}}, X_{t_{2}}, X_{t_{3}}, \ldots\right) \in B\right)=\mathcal{P}\left(\left(X_{t_{1}+s}, X_{t_{2}+s}, X_{t_{3}+s}, \ldots\right) \in B\right)$.

- Para $\forall n \in \mathbb{N}$ temos que $F_{X_{t_{1}}, . ., X_{t_{n}}}\left(x_{1}, \ldots x_{n}\right)=F_{X_{t_{1}+s}, . ., X_{t_{n}+s}}\left(x_{1}, \ldots x_{n}\right)$.

- Para $\forall n \in \mathbb{N}$ temos que $\mathcal{P}\left(X_{t_{1}}<x_{1}, \ldots, X_{t_{n}}<x_{n}\right)=\mathcal{P}\left(X_{t_{1}+s}<x_{1}, \ldots, X_{t_{n}+s}<x_{n}\right)$.

- Para $\forall A \in \mathcal{B}\left(\mathbb{R}^{n}\right) n \in \mathbb{N}$ temos que $\mathcal{P}\left(\left(X_{t_{1}}, \ldots, X_{t_{n}}\right)^{-1}(A)\right)=\mathcal{P}\left(\left(X_{t_{1}+s}, \ldots, X_{t_{n}+s}\right)^{-1}(A)\right)$.

DEFINIÇÃo 4.1.9: Um processo estocástico $\left\{X_{t}\right\}_{t \in \mathbb{R}}$ é simétrico se para qualquer seqüência de números reais $\left\{t_{i}\right\}_{i=1}^{\infty}$ temos que o processo $X_{t_{1}}, X_{t_{2}}, \ldots$ tem a mesma distribuição de probabilidade de $X_{-t_{1}}, X_{-t_{2}}, \ldots$ Vale assim as propriedades acima com $t_{i+s} \rightarrow-t_{i}$.

Uma última caracterização de processos estocásticos com mesma distribuição de probabilidade será mostrada abaixo. Elas nos será muito importante para o estudo dos operadores $U(t)$ e $R$, que serão definidos adiante, e será usada freqüentemente nas demonstrações seguintes. De certa forma, este Teorema vai justificar a importância dos processos estocásticos simétricos e estacionários para nós, ao indicar que o valor de certas integrais é invariante.

TEOREMA 4.1.10: Dois processos estocásticos $\left\{X_{n}\right\}_{n=1}^{\infty}$ e $\left\{Y_{n}\right\}_{n=1}^{\infty}$ têm a mesma distribuição de probabilidades se, e somente se, qualquer função $F:\left(\mathbb{R}^{n}, \mathcal{B}\left(\mathbb{R}^{n}\right)\right) \rightarrow(\mathbb{R}, \mathcal{B}(\mathbb{R}))$ mensurável (para qualquer $n \in \mathbb{N}$ ) satisfaz:

$$
\int_{\Omega} F\left(X_{1}, \ldots, X_{n}\right) d \mu=\int_{\Omega^{\prime}} F\left(Y_{1}, \ldots, Y_{n}\right) d \mu^{\prime}
$$

em que $X_{i}:(\Omega, \mathcal{F}, \mu) \rightarrow(\mathbb{R}, \mathcal{B}(\mathbb{R}))$ e $Y_{i}:\left(\Omega^{\prime}, \mathcal{F}^{\prime}, \mu^{\prime}\right) \rightarrow(\mathbb{R}, \mathcal{B}(\mathbb{R}))$ para $i=1,2,3, \ldots$

Prova:

$(\Leftarrow)$ Se tivermos a igualdade entre as integrais então dado $A \in \mathcal{B}\left(\mathbb{R}^{n}\right)$ temos:

$$
\begin{aligned}
& \mu\left(\left(X_{1}, \ldots, X_{n}\right)^{-1}(A)\right)=\int_{\Omega} \chi_{\left(X_{1}, \ldots, X_{n}\right)^{-1}(A)}(\omega) d \mu=\int_{\Omega} \chi_{A}\left(X_{1}(\omega), \ldots, X_{n}(\omega)\right) d \mu \\
& =\int_{\Omega^{\prime}} \chi_{A}\left(Y_{1}(\omega), \ldots, Y_{n}(\omega)\right) d \mu^{\prime}=\int_{\Omega^{\prime}} \chi_{\left(Y_{1}, \ldots, Y_{n}\right)^{-1}(A)}(\omega) d \mu^{\prime}=\mu^{\prime}\left(\left(Y_{1}, \ldots, Y_{n}\right)^{-1}(A)\right) .
\end{aligned}
$$

Como isto vale para qualquer que seja $n \in \mathbb{N}$ e $A \in \mathcal{B}\left(\mathbb{R}^{n}\right)$ então concluímos que $\left\{X_{n}\right\}_{n=1}^{\infty}$ e $\left\{Y_{n}\right\}_{n=1}^{\infty}$ tem a mesma distribuição de probabilidade (pois suas funções de distribuição são as mesmas).

$(\Rightarrow)$ Se os dois processos têm a mesma distribuição de probabilidade então consideremos F uma função mensurável simples. Logo $F\left(x_{1}, \ldots, x_{n}\right)=\sum_{k=1}^{N} a_{k} \chi_{A_{k}}\left(x_{1}, \ldots, x_{n}\right)$. Assim obtemos que $\int_{\Omega} F\left(X_{1}, \ldots, X_{n}\right) d \mu=\int_{\Omega} \sum_{k=1}^{N} a_{k} \chi_{A_{k}}\left(X_{1}, \ldots, X_{n}\right) d \mu=\int_{\Omega} \sum_{k=1}^{N} a_{k} \chi_{\left(X_{1}, \ldots, X_{n}\right)^{-1}\left(A_{k}\right)} d \mu=$ 
$\sum_{k=1}^{N} a_{k} \mu\left(\left(X_{1}, \ldots, X_{n}\right)^{-1}\left(A_{k}\right)\right)=\sum_{k=1}^{N} a_{k} \mu^{\prime}\left(\left(Y_{1}, \ldots, Y_{n}\right)^{-1}\left(A_{k}\right)\right)=\int_{\Omega^{\prime}} \sum_{k=1}^{N} a_{k} \chi_{\left(Y_{1}, \ldots, Y_{n}\right)^{-1}\left(A_{k}\right)} d \mu^{\prime}=$ $\int_{\Omega^{\prime}} \sum_{k=1}^{N} a_{k} \chi_{A_{k}}\left(Y_{1}, \ldots, Y_{n}\right) d \mu^{\prime}=\int_{\Omega^{\prime}} F\left(Y_{1}, \ldots, Y_{n}\right) d \mu^{\prime}$

Se $F$ é positivo então existe $F_{n}$ simples tal que $F_{n} \uparrow F$. Logo

$$
\begin{aligned}
& \int_{\Omega} F\left(X_{1}, \ldots, X_{n}\right) d \mu=\lim _{n \rightarrow \infty} \int_{\Omega} F_{n}\left(X_{1}, \ldots, X_{n}\right) d \mu= \\
& =\lim _{n \rightarrow \infty} \int F_{n}\left(Y_{1}, \ldots, Y_{n}\right) d \mu^{\prime}=\int_{\Omega^{\prime}} F\left(Y_{1}, \ldots, Y_{n}\right) d \mu^{\prime} .
\end{aligned}
$$

Se $F$ é qualquer então podemos fazer $F=F^{+}-F^{-}$. Como

$$
\int_{\Omega} F^{ \pm}\left(X_{1}, \ldots, X_{n}\right) d \mu=\int_{\Omega^{\prime}} F^{ \pm}\left(Y_{1}, \ldots, Y_{n}\right) d \mu^{\prime}
$$

temos que $F\left(X_{1}, \ldots, X_{n}\right)$ é integrável se, e somente se, $F\left(Y_{1}, \ldots, Y_{n}\right)$ é integrável. Neste caso concluímos que $\int_{\Omega} F\left(X_{1}, \ldots, X_{n}\right) d \mu=\int_{\Omega^{\prime}} F\left(Y_{1}, \ldots, Y_{n}\right) d \mu^{\prime}$

Vamos agora nos restringir ao estudo de processos estocásticos em que a $\sigma$-álgebra $\mathcal{F}$ é a menor $\sigma$-álgebra que torna todos os $X_{t}$ mensuráveis, ou seja, $\mathcal{F}=\sigma$-álgebra $\left[X_{t}^{-1}(A), A \in \mathcal{B}(\mathbb{R})\right.$ e $t \in \mathbb{R}]$. Vamos agora denotar a $\sigma$-álgebra gerada por funções $X_{t_{1}}, \ldots, X_{t_{n}}$ por $\mathcal{B}\left(X_{t_{1}}, \ldots, X_{t_{n}}\right)$ e a gerada por $X_{t}$, com $t \in \mathbb{R}$, por $\mathcal{B}\left(X_{t}, t \in \mathbb{R}\right)$. Em relação a estes processos, há propriedades muito interessantes e que serão usadas adiante. Por isto vamos enunciá-las e prová-las a seguir $(\operatorname{ver}[4])$ :

ProposiçÃo 4.1.11 [4]: Seja $\mathcal{B}\left(X_{1}, \ldots, X_{n}\right)$ a $\sigma$-álgebra gerada por $X_{1}, \ldots, X_{n}$ variáveis aleatórias de $\Omega \rightarrow \mathbb{R}$. Então se $\Lambda \in \mathcal{B}\left(X_{1}, \ldots, X_{n}\right)$ existe um $A \in \mathcal{B}\left(\mathbb{R}^{n}\right)$ tal que $\Lambda=\{x \in$ $\left.\Omega ;\left(X_{1}(x), \ldots, X_{n}(x)\right) \in A\right\}$.

Prova: Seja $\mathcal{F}$ a coleção de conjunto $\mathcal{F}=\left\{\Lambda \in \Omega ; \Lambda=\left(X_{1}, \ldots, X_{n}\right)^{-1}(A) \operatorname{com} A \in \mathcal{B}\left(\mathbb{R}^{n}\right)\right\}$.

$\mathcal{F}$ é uma $\sigma$-álgebra, pois

1) $\Omega=\left(X_{1}, \ldots, X_{n}\right)^{-1}\left(\mathbb{R}^{n}\right) \in \mathcal{F}$.

2) Se $\Lambda \in \mathcal{F}$ então $\Lambda^{c}=\left(X_{1}, \ldots, X_{n}\right)^{-1}(A)^{c}=\left(X_{1}, \ldots, X_{n}\right)^{-1}\left(A^{c}\right) \in \mathcal{F}$.

3) Se $\Lambda_{n} \in \mathcal{F}$ então $\cup_{n=1}^{\infty} \Lambda_{n}=\cup_{n=1}^{\infty}\left(X_{1}, \ldots, X_{n}\right)^{-1}\left(A_{n}\right)=\left(X_{1}, \ldots, X_{n}\right)^{-1}\left(\cup_{n=1}^{\infty} A_{n}\right) \in \mathcal{F}$, em que $\Lambda_{n}=\left(X_{1}, \ldots, X_{n}\right)^{-1}\left(A_{n}\right)$.

$X_{j}$ é mensurável em $\mathcal{F}$ para qualquer que seja $j=1, \ldots, n$, pois se $A \in \mathcal{B}(\mathbb{R})$ então $X_{j}^{-1}(A)=$ $\left(X_{1}, \ldots, X_{j}, \ldots, X_{n}\right)^{-1}(\mathbb{R} \times \ldots \times A \times \ldots \times \mathbb{R}) \in \mathcal{F} . \operatorname{Assim} \mathcal{B}\left(X_{1}, \ldots, X_{n}\right) \subset \mathcal{F}$

Sabe-se que se $F:\left(\mathbb{R}^{n}, \mathcal{B}\left(\mathbb{R}^{n}\right)\right) \rightarrow(\mathbb{R}, \mathcal{B}(\mathbb{R}))$ é mensurável e $X_{j}:\left(\Omega, \mathcal{B}\left(X_{1}, \ldots, X_{n}\right)\right) \rightarrow$ $(\mathbb{R}, \mathcal{B}(\mathbb{R}))$ é mensurável para qualquer que seja $j=1, \ldots, n$. Então a função dada por $F\left(X_{1}, \ldots, X_{n}\right)$ : $\left(\Omega, \mathcal{B}\left(X_{1}, . ., X_{n}\right)\right) \rightarrow(\mathbb{R}, \mathcal{B}(\mathbb{R}))$ é mensurável [31].

Logo dado $A \in \mathcal{B}\left(\mathbb{R}^{n}\right)$, então $\chi_{A}:\left(\mathbb{R}^{n}, \mathcal{B}\left(\mathbb{R}^{n}\right)\right) \rightarrow(\mathbb{R}, \mathcal{B}(\mathbb{R}))$ é mensurável. Assim, a função $\chi_{A}\left(X_{1}, \ldots, X_{n}\right):\left(\Omega, \mathcal{B}\left(X_{1}, \ldots, X_{n}\right)\right) \rightarrow(\mathbb{R}, \mathcal{B}(\mathbb{R}))$ é mensurável. Desta forma notamos que $\chi_{A}\left(X_{1}, \ldots, X_{n}\right)^{-1}(\{1\})=\left(X_{1}, \ldots, X_{n}\right)^{-1}(A) \in \mathcal{B}\left(X_{1}, \ldots, X_{n}\right)$ e, portanto, $\mathcal{F} \subset \mathcal{B}\left(X_{1}, \ldots, X_{n}\right)$. Concluímos então que $\mathcal{B}\left(X_{1}, \ldots, X_{n}\right)=\mathcal{F}$. 
ProposiçÃo 4.1 .12 [4]: Seja $X_{1}, \ldots, X_{n}$ variáveis aleatórias. Então $F:\left(\Omega, \mathcal{B}\left(X_{1}, \ldots, X_{n}\right)\right) \rightarrow$ $(\mathbb{R}, \mathcal{B}(\mathbb{R}))$ é mensurável se, e somente se, $F=G\left(X_{1}, \ldots, X_{n}\right)$ em que $G:\left(\mathbb{R}^{n}, \mathcal{B}\left(\mathbb{R}^{n}\right)\right) \rightarrow$ $(\mathbb{R}, \mathcal{B}(\mathbb{R}))$ é mensurável.

Prova: $(\Leftarrow)$ Vem da observação acima [31].

$(\Rightarrow)$ Seja $\Lambda_{j m}=\left\{\frac{j}{2^{m}}<F \leq \frac{j+1}{2^{m}}\right\} \in \mathcal{B}\left(X_{1}, \ldots, X_{n}\right)$. Existe então $A_{j m}^{\prime} \in \mathcal{B}\left(\mathbb{R}^{n}\right)$ tal que $\Lambda_{j m}=\left(X_{1}, \ldots, X_{n}\right)^{-1}\left(A_{j m}^{\prime}\right)$.

Sabemos então que $\Lambda_{j m}$ são disjuntos. Logo seja $A_{j m}=A_{j m}^{\prime}-\left[A_{j m}^{\prime} \cap\left(\cup_{i \neq j} A_{i m}^{\prime}\right)\right]$. As$\operatorname{sim} \Lambda_{j m}=\left(X_{1}, \ldots, X_{n}\right)^{-1}\left(A_{j m}\right)$ pois se $x \in \Lambda_{j m}$ então $\left(X_{1}, \ldots, X_{n}\right)(x) \in A_{j m}^{\prime}$. Mas se $\left(X_{1}, \ldots, X_{n}\right)(x) \in A_{j m}^{\prime} \cap\left(\cup_{i \neq j} A_{i m}^{\prime}\right)$ então $\left(X_{1}, \ldots, X_{n}\right)(x) \in A_{k m}$ para algum $k \neq j$. Logo $x \in$ $\Lambda_{k m}$. Como $\Lambda_{j m} \cap \Lambda_{k m}=\emptyset$, temos uma contradição. Logo $x \in A_{j m}^{\prime}-\left[A_{j m}^{\prime} \cap\left(\cup_{i \neq j} A_{i m}^{\prime}\right)\right]=A_{j m}$. Agora seja $F_{m}(\omega)=\frac{j}{2^{m}}$, para $\omega \in \Lambda_{j m} j=0, \pm 1, \ldots$ Temos então que $F_{m}(\omega)=G_{m}\left(X_{1}, \ldots, X_{n}\right)$ em que:

$$
G_{m}\left(\xi_{1}, \ldots, \xi_{n}\right)=\left\{\begin{array}{c}
\frac{j}{2^{m}}, \text { se }\left(\xi_{1}, \ldots, \xi_{n}\right) \in A_{j m} \\
0, \text { de outra forma }
\end{array} .\right.
$$

Assim na imagem de $\left(X_{1}, \ldots, X_{n}\right)(\Omega)$ temos

$$
\lim _{m \rightarrow \infty} G_{m}\left(X_{1}(\omega), \ldots, X_{n}(\omega)\right)=\lim _{m \rightarrow \infty} F_{m}(\omega)=F(\omega) .
$$

Logo definamos $G\left(\xi_{1}, \ldots, \xi_{n}\right)=\limsup _{m \rightarrow \infty} G_{m}\left(\xi_{1}, \ldots, \xi_{n}\right)$ (temos que fazer isto, pois só garantíamos que existe o limite na imagem da função $\left(X_{1}, \ldots, X_{n}\right)(\Omega)$ ). Assim $G$ é mensurável e $G\left(X_{1}, \ldots, X_{n}\right)(\omega)=F(\omega)$

ProposiçÃo 4.1.13 [4]: Seja $\left\{X_{t}, t \in T\right\}$ um processo estocástico num espaço de probabilidade $\left(\Omega, \mathcal{B}\left(X_{t}, t \in T\right), \mu\right)$.

1) Se $T$ não é enumerável e $\Lambda \in \mathcal{B}\left(X_{t}, t \in T\right)$, então existe $S \subset T$ enumerável tal que $\Lambda \in \mathcal{B}\left(X_{t}, t \in S\right)$.

2) Se $F:\left(\Omega, \mathcal{B}\left(X_{t}, t \in T\right)\right) \rightarrow(\mathbb{R}, \mathcal{B}(\mathbb{R}))$ é mensurável, então existe $S \subset T$ enumerável tal que $F:\left(\Omega, \mathcal{B}\left(X_{t}, t \in S\right)\right) \rightarrow(\mathbb{R}, \mathcal{B}(\mathbb{R}))$ é mensurável.

Prova:

1) Seja $\mathcal{G}=\left\{\Lambda \in \mathcal{B}\left(X_{t}, t \in T\right) ; \exists S \subset T\right.$ enumerável tal que $\left.\Lambda \in \mathcal{B}\left(X_{t}, t \in S\right)\right\}$. Então $\mathcal{G}$ é $\sigma$-álgebra, pois

(i) $\Omega \in \mathcal{B}\left(X_{t}\right)$ para qualquer que seja $t \in T$ o que implica que $\Omega \in \mathcal{G}$.

(ii) $\Lambda \in \mathcal{G}$ o que implica que $\Lambda \in \mathcal{B}\left(X_{t}, t \in S\right)$ logo que $\Lambda^{c} \in \mathcal{B}\left(X_{t}, t \in S\right)$ e $\Lambda^{c} \in \mathcal{G}$.

(iii) $\operatorname{se}\left\{\Lambda_{n}\right\}_{n \in \mathbb{N}} \in \mathcal{G}$ então $\Lambda_{n} \in \mathcal{B}\left(X_{t}, t \in S_{n}\right)$, logo $\Lambda_{n} \in \mathcal{B}\left(X_{t}, t \in \cup_{n=1}^{\infty} S_{n}\right)$ e $\cup_{n=1}^{\infty} \Lambda_{n} \in$ $\mathcal{B}\left(X_{t}, t \in \cup_{n=1}^{\infty} S_{n}\right)$.

Também vemos que $\mathcal{G} \subset \mathcal{B}\left(X_{t}, t \in T\right)$. Mas se $\Lambda \in \mathcal{B}\left(X_{t}\right)$ então $\Lambda \in \mathcal{G}$. Logo $X_{t}:(\Omega, \mathcal{G}) \rightarrow$ $(\mathbb{R}, \mathcal{B}(\mathbb{R}))$ é mensurável para qualquer que seja $t \in T$. Assim $\mathcal{B}\left(X_{t}, t \in T\right) \subset \mathcal{G}$. Portanto $\mathcal{G}=\mathcal{B}\left(X_{t}, t \in T\right)$.

2)Seja $r \in \mathbb{Q}$ então $\left.\left.F^{-1}(]-\infty, r\right]\right) \in \mathcal{B}\left(X_{t}, t \in S_{r}\right)$. Assim dado $q \in \mathbb{Q}$ então $\left.\left.F^{-1}(]-\infty, q\right]\right) \in$ 
$\mathcal{B}\left(X_{t}, t \in \cup_{r \in \mathbb{Q}} S_{r}\right) . \quad$ Logo $F:\left(\Omega, \mathcal{B}\left(X_{t}, t \in \cup_{r \in \mathbb{Q}} S_{r}\right)\right) \rightarrow(\mathbb{R}, \mathcal{B}(\mathbb{R}))$ é mensurável e $\cup_{r \in \mathbb{Q}} S_{r}$ é enumerável. Note que a mesma prova vale se substituirmos $\mathbb{R}$ por um espaço topológico segundo contável, para tanto basta substituir ] $-\infty, r$ ] por conjuntos de uma base enumerável deste espaço.

Sabendo dos fatos anteriores, podemos mostrar que as funções do tipo $F\left(X_{1}, \ldots, X_{n}\right)$ são $L_{p}$ -densas em um processo estocástico. Para tanto precisamos do seguinte lema.

LEMA 4.1 .14 [21]: Seja $(\Omega, \mathcal{F}, \mu)$ um espaço de probabilidade e $\mathcal{B} \subset \mathcal{F}, \mathcal{B}$ uma álgebra que gera $\mathcal{F}$. Seja

$$
\mathbb{H}=\left\{h: \Omega \rightarrow \mathbb{R}, h=\sum_{i=1}^{n} a_{i} \chi_{B_{i}}, a_{i} \in \mathbb{R} e B_{i} \in \mathcal{B} \text { são disjuntos }\right\}
$$

Assim $\mathbb{H}$ é denso em $L^{p}(\Omega, \mathcal{F}, \mu)$ para $1 \leq p<\infty$.

Prova: Seja $\mathbb{W}=\left\{h: \Omega \rightarrow \mathbb{R} ; h=\sum_{i=1}^{n} a_{i} \chi_{A_{i}}\right.$ em que $a_{i} \in \mathbb{R}$ e $A_{i} \in \mathcal{F}, A_{i}$ são disjuntos $\}$. Então $\mathbb{W}$ é denso em $L^{p}(\Omega, \mathcal{F}, \mu)$. Para vermos isto, seja dada $f \in L^{p}(\Omega, \mathcal{F}, \mu)$. Assim $f=f^{+}-$ $f^{-}$e existem seqüências $\left\{f_{n}^{+}\right\}$e $\left\{f_{n}^{-}\right\} \in \mathbb{W}$ tais que $f_{n}^{+} \nearrow f^{+} f_{n}^{-} \nearrow f^{-}$. Logo como $f_{n}^{+} \leq f^{+}$e $f_{n}^{-} \leq f^{-}$concluímos que $\left|f_{n}\right|=\left|f_{n}^{+}-f_{n}^{-}\right|=f_{n}^{+}+f_{n}^{-} \leq f^{+}+f^{-}=|f|$. Assim $f_{n} \rightarrow f$ e $\left|f_{n}\right| \leq|f|$. Logo $\left|f-f_{n}\right|^{p} \leq\left(|f|+\left|f_{n}\right|\right)^{p} \leq 2^{p}|f|$. Desta forma temos que $\left|f-f_{n}\right|^{p}(x) \rightarrow 0$ e $\left|f-f_{n}\right|^{p} \leq 2^{p}|f|$ (logo a função é dominada). Portanto, $\lim _{n \rightarrow \infty} \int_{\Omega}\left|f-f_{n}\right|^{p} d \mu=\int_{\Omega} \lim _{n \rightarrow \infty}\left|f-f_{n}\right|^{p} d \mu=0$. Logo $\mathbb{W}$ é denso em $L^{p}(\Omega, \mathcal{F}, \mu)$.

Agora mostraremos que $\mathbb{H}$ é denso em $\mathbb{W}$.

Seja $h \in \mathbb{W}$ tal que $h=\sum_{i=1}^{n} a_{i} \chi_{A_{i}}$. Pelo Teorema da aproximação temos que existe $B_{i} \in \mathcal{B}$ (pois $\mu$ é finita, $\left.\operatorname{logo} \mu\right|_{\mathcal{B}}$ é $\sigma$-finita e $\mu\left(A_{i}\right)<\infty$, ver [31] lema 5.2.15) tal que $\mu\left(A_{i} \triangle B_{i}\right)<$ $\left(\frac{\epsilon}{\sum_{i=1}^{n}\left|a_{i}\right|}\right)^{p}$.

Seja $h^{\prime}=\sum_{i=1}^{n} a_{i} \chi_{B_{i}}$ então

$$
\begin{gathered}
\left\|h-h^{\prime}\right\|_{p}=\|\left.\sum_{i=1}^{n} a_{i}\left(\chi_{A_{i}}-\chi_{B_{i}}\right)\right|_{p} \leq \sum_{i=1}^{n}\left|a_{i}\right||| \chi_{A_{i}}-\chi_{B_{i}}||_{p}=\sum_{i=1}^{n}\left|a_{i}\right|\left(\int\left|\chi_{A_{i}}-\chi_{B_{i}}\right|^{p} d \mu\right)^{\frac{1}{p}} \\
=\sum_{i=1}^{n}\left|a_{i}\right|\left(\int \chi_{A_{i} \triangle B_{I}}^{p} d \mu\right)^{\frac{1}{p}} \leq \sum_{i=1}^{n}\left|a_{i}\right|\left[\left(\frac{\epsilon}{\sum_{i=1}^{n}\left|a_{i}\right|}\right)^{p}\right]^{\frac{1}{p}}=\sum_{i=1}^{n}\left|a_{i}\right| \frac{\epsilon}{\sum_{i=1}^{n}\left|a_{i}\right|}=\epsilon .
\end{gathered}
$$

Na terceira igualdade usou-se a relação $\left|\chi_{A_{i}}-\chi_{B_{i}}\right|=\chi_{A_{i} \triangle B_{I}}$. Agora basta escrever $h^{\prime}$ na forma canônica, que é possível já que para isto basta fazer intersecções finitas e diferenças (logo é possível de fazer na álgebra $\mathcal{B}$ ). Vemos, então, que $h^{\prime} \in \mathbb{H}$.

Agora podemos enunciar o Teorema principal. Este Teorema nos permitirá estudar os ope- 
radores definidos em $L^{p}\left(\Omega, \mathcal{B}\left(X_{t}, t \in \mathbb{R}\right), \mu\right)$, que serão muito importante para nós.

TeOrema 4.1.15: Seja $\left\{X_{t}\right\}_{t \in \mathbb{R}}$ um processo estocástico de $\left(\Omega, \mathcal{B}\left(X_{t}, t \in \mathbb{R}\right), \mu\right)$ em $(\mathbb{R}, \mathcal{B}(\mathbb{R}))$. Então o conjunto $\mathcal{A}=\left\{G:\left(\Omega, \mathcal{B}\left(X_{t}, t \in \mathbb{R}\right), \mu\right) \rightarrow(\mathbb{R}, \mathcal{B}(\mathbb{R})) ; G\right.$ mensurável, limitado e da forma $G=F\left(X_{t_{1}}, \ldots, X_{t_{n}}\right)$ em que $F:\left(\mathbb{R}^{n}, \mathcal{B}\left(\mathbb{R}^{n}\right)\right) \rightarrow(\mathbb{R}, \mathcal{B}(\mathbb{R}))$ é mensurável $\}$ é denso no conjunto $L^{p}\left(\Omega, \mathcal{B}\left(X_{t}, t \in \mathbb{R}\right), \mu\right)$.

Prova:

Seja $G \in L^{p}\left(\Omega, \mathcal{B}\left(X_{t}, t \in \mathbb{R}\right), \mu\right)$. Logo existe $S$ enumerável tal que $S \subset \mathbb{R}$ e que $G$ : $\left(\Omega, \mathcal{B}\left(X_{t}, t \in S\right), \mu\right) \rightarrow(\mathbb{R}, \mathcal{B}(\mathbb{R}))$ é mensurável, pela proposição 4.1.13 mostrada acima. Logo $G \in L^{p}\left(\Omega, \mathcal{B}\left(X_{t}, t \in S\right), \mu\right)$. Seja $S=\left\{t_{1}, t_{2}, t_{3}, \ldots\right\}$. Sabemos que $\mathcal{B}\left(X_{t}, t \in S\right)$ é a menor $\sigma$ álgebra que torna todos os $X_{t}$ mensuráveis. Assim $\mathcal{B}\left(X_{1}\right), \mathcal{B}\left(X_{1}, X_{2}\right), \ldots \in \mathcal{B}\left(X_{t}, t \in S\right)$. Logo $\cup_{n=1}^{\infty} \mathcal{B}\left(X_{t_{1}}, \ldots, X_{t_{n}}\right) \subset \mathcal{B}\left(X_{t}, t \in S\right)$. Ao mesmo tempo $X_{t_{j}} \in \mathcal{B}\left(X_{t_{1}}, \ldots, X_{t_{j}}\right)$. Logo $X_{t_{j}} \in \sigma$ álgebra $\left[\cup_{n=1}^{\infty} \mathcal{B}\left(X_{t_{1}}, \ldots, X_{t_{n}}\right)\right]$, o que implica que $\mathcal{B}\left(X_{t}, t \in S\right) \subset \sigma$-álgebra $\left[\cup_{n=1}^{\infty} \mathcal{B}\left(X_{t_{1}}, . ., X_{t_{n}}\right)\right]$. Concluímos, então, que $\mathcal{B}\left(X_{t}, t \in S\right)$ é igual a $\sigma$-álgebra $\left[\cup_{n=1}^{\infty} \mathcal{B}\left(X_{t_{1}}, \ldots, X_{t_{n}}\right)\right]$

Assim $\cup_{n=1}^{\infty} \mathcal{B}\left(X_{t_{1}}, \ldots, X_{t_{n}}\right)$ é uma álgebra que gera $\mathcal{B}\left(X_{t}, t \in S\right)$. Logo, pelo lema 4.1.14 mostrado, temos que dado $G \in L^{p}\left(\Omega, \mathcal{B}\left(X_{t}, t \in S\right), \mu\right)$ existe $h=\sum_{n=1}^{N} a_{n} \chi_{A_{n}}$ em que $A_{n} \in \cup_{n=1}^{\infty} \mathcal{B}\left(X_{t_{1}}, \ldots, X_{t_{n}}\right)$ tal que $\|G-h\|_{p}<\epsilon$. Porém $A_{n} \in \mathcal{B}\left(X_{t_{1}}, . ., X_{t_{m(n)}}\right)$ o que implica (ver proposição 4.1.11) que $A_{n}=\left(X_{t_{1}}, \ldots, X_{t_{m(n)}}\right)^{-1}\left(B_{n}\right)$ em que $B_{n} \in \mathcal{B}\left(\mathbb{R}^{m}\right)$. Logo $h=\sum_{n=1}^{N} a_{n} \chi_{\left(X_{t_{1}}, \ldots, X_{t_{m(n)}}\right)^{-1}\left(B_{n}\right)}$. Seja $M=\max \{m(n) ; n=1, \ldots, N\}$. Sabemos então que $\left(X_{t_{1}}, \ldots, X_{t_{m(n)}}\right)^{-1}\left(B_{n}\right)$ pode ser escrito como $\left(X_{t_{1}}, \ldots, X_{t_{m(n)}}, X_{t_{m(n)+1}}, \ldots, X_{M}\right)^{-1}\left(B_{n} \times \mathbb{R}^{M-t_{m(n)}}\right)$. Assim $h=\sum_{n=1}^{N} a_{n} \chi_{\left(X_{t_{1}}, \ldots, X_{t_{n}}\right)^{-1}\left(B_{n}^{\prime}\right)}=\sum_{n=1}^{N} a_{n} \chi_{B_{n}^{\prime}}\left(X_{t_{1}}, \ldots, X_{t_{M}}\right)$, em que $B_{n}^{\prime}=B_{n} \times \mathbb{R}^{M-t_{m(n)}}$ e $\chi_{B_{n}^{\prime}} \in \mathcal{B}\left(\mathbb{R}^{M}\right)$. Logo $h=F\left(X_{t_{1}}, \ldots, X_{t_{M}}\right)$, em que $F\left(x_{1}, \ldots, x_{M}\right)=\sum_{n=1}^{N} a_{n} \chi_{B_{n}^{\prime}}\left(x_{1}, \ldots, x_{M}\right)$ e, como $F$ é mensurável em $\mathcal{B}\left(\mathbb{R}^{M}\right)$ e limitado, concluímos a demonstração do Teorema.

Agora que já estudamos aspectos básicos da teoria de processos estocásticos, estamos em condições de definir e estudar dois operadores que desempenharão um papel de grande importância no trabalho.

DEFINIÇÃo 4.1.16: Definimos os operadores $U(t)$ e $R$ agindo no conjunto das funções mensuráveis de $\left(\Omega, \mathcal{B}\left(X_{t}, t \in \mathbb{R}\right), \mu\right)$ em $(\mathbb{R}, \mathcal{B}(\mathbb{R}))$ que têm a forma $F\left(X_{t_{1}}, \ldots, X_{t_{n}}\right)$, em que $F$ é limitado e mensurável de $\left(\mathbb{R}^{n}, \mathcal{B}\left(\mathbb{R}^{n}\right)\right)$ em $(\mathbb{R}, \mathcal{B}(\mathbb{R}))$ por:

$$
\begin{gathered}
U(t) F\left(X_{t_{1}}, \ldots, X_{t_{n}}\right)=F\left(X_{t_{1}+t}, \ldots, X_{t_{n}+t}\right) \\
R F\left(X_{t_{1}}, \ldots, X_{t_{n}}\right)=F\left(X_{-t_{1}}, \ldots, X_{-t_{n}}\right) .
\end{gathered}
$$


1. Se o processo é estacionário então $U(t)$ se estende a grupo uniparamétrico de isometrias em $L^{p}\left(\Omega, \mathcal{B}\left(X_{t}, t \in \mathbb{R}\right), \mu\right)$

2. Se o processo é simétrico então $R$ se estende a uma isometria em $L^{p}\left(\Omega, \mathcal{B}\left(X_{t}, t \in \mathbb{R}\right), \mu\right)$

Prova:

Se o processo é estacionário então

$$
\left\|U(t) F\left(X_{t_{1}}, \ldots, X_{t_{n}}\right)\right\|_{p}=\left\|F\left(X_{t_{1}+t}, . ., X_{t_{n}+t}\right)\right\|_{p}=\left\|F\left(X_{t_{1}}, \ldots, X_{t_{n}}\right)\right\|_{p}
$$

pelo Teorema 4.1.10. Logo $U(t)$ é contínuo e definido num domínio denso de $L^{p}\left(\Omega, \mathcal{B}\left(X_{t}, t \in\right.\right.$ $\mathbb{R}), \mu$ ). Pelo Teorema B.L.T. ([6] pag.9) $U(t)$ pode ser estendido unicamente para um operador limitado e dado $F \in L^{p}\left(\Omega, \mathcal{B}\left(X_{t}, t \in \mathbb{R}\right), \mu\right)$ temos

$$
\|U(t) F\|=\lim _{n \rightarrow \infty}\left\|U(t) F_{n}\right\|=\lim _{n \rightarrow \infty}\left\|F_{n}\right\|=\|F\| .
$$

Logo $U(t)$ é isometria.

Se o processo é simétrico então

$$
\left\|R F\left(X_{t_{1}}, \ldots, X_{t_{n}}\right)\right\|_{p}=\left\|F\left(X_{-t_{1}}, \ldots, X_{-t_{n}}\right)\right\|=\left\|F\left(X_{t_{1}}, \ldots, X_{t_{n}}\right)\right\|_{p}
$$

pelo Teorema 4.1.10. Novamente pelo Teorema BLT, $R$ se estende de forma única a uma isometria.

Estaremos interessados agora em estender o resultado do Teorema 4.1.17, provando que $R$ e $U(t)$ se estendem a grupos de ${ }^{*}$-automorfismos em $L^{\infty}(Q, \Sigma, \mu)$. Para tanto provaremos resultados intermediários que serão interessantes no estudo da referência [7] que será feito nas próximas seções. Inicialmente vamos provar um resultado simples.

ProposiçÃo 4.1.18: Seja $A \in \Sigma=\mathcal{B}\left(X_{t}, t \in[-\beta / 2, \beta / 2]\right)$. Então existe uma seqüência de funções que convergem a $\chi_{A}$, no sentido de $L^{1}(Q, \Sigma, \mu)$. Estas funções assumem apenas valores 0 e 1 e são da forma $H\left(X_{t_{1}}, \ldots, X_{t_{n}}\right)$, em que $H:\left(\mathbb{R}^{n}, \mathcal{B}\left(\mathbb{R}^{n}\right)\right) \rightarrow(\mathbb{R}, \mathcal{B}(\mathbb{R}))$ é uma função limitada.

Prova: Sabemos que existem funções $F_{n}\left(X_{t_{1}}, \ldots, X_{t_{m(n)}}\right)$ que convergem a $\chi_{A}$ quando n vai ao infinito, no sentido de $L^{1}$, devido ao Teorema 4.1.15. Podemos então definir as funções $G_{n}$ por

$$
G_{n}(x)=\left\{\begin{array}{l}
1 \text { se } F_{n}\left(X_{t_{1}}(x), \ldots, X_{t_{m(n)}}(x)\right) \in\left[\frac{1}{2},+\infty\right] \\
0 \text { se } F_{n}\left(X_{t_{1}}(x), \ldots, X_{t_{m(n)}}(x)\right) \in\left[-\infty, \frac{1}{2}[\right.
\end{array} .\right.
$$

Notamos que o conjunto $F_{n}\left(X_{t_{1}}(x), \ldots, X_{t_{m(n)}}(x)\right) \in\left[\frac{1}{2},+\infty\right]=\left(X_{t_{1}}(x), \ldots, X_{t_{m(n)}}(x)\right)^{-1} \circ$ $F_{n}^{-1}\left[\frac{1}{2},+\infty\right]$ que pertence a $\mathcal{B}\left(X_{t_{1}}, \ldots, X_{t_{m(n)}}\right)$, o mesmo para o conjunto $F_{n}\left(X_{t_{1}}(x), \ldots, X_{t_{m(n)}}(x)\right) \in$ 
$\left[-\infty, \frac{1}{2}\right]$. Logo pela proposição 4.1 .12 , concluímos que $G_{n}=H_{n}\left(X_{t_{1}}, \ldots, X_{t_{m(n)}}\right)$, em que evidentemente podemos escolher $H_{n}$ assumindo apenas valores 0 e 1 . Mostraremos agora que $G_{n}$ vai a $\chi_{A}$ na norma de $L^{1}$. Definamos inicialmente os conjuntos

$$
\begin{aligned}
& \mathcal{E}=\left\{F_{n}\left(X_{t_{1}}(x), \ldots, X_{t_{m(n)}}(x)\right) \in\left[\frac{1}{2},+\infty\right]\right\} \cap\left\{\chi_{A}(x)=0\right\} \\
& \mathcal{F}=\left\{F _ { n } ( X _ { t _ { 1 } } ( x ) , \ldots , X _ { t _ { m ( n ) } } ( x ) ) \in \left[-\infty, \frac{1}{2}[\} \cap\left\{\chi_{A}(x)=1\right\}\right.\right.
\end{aligned} .
$$

Assim concluímos que

$$
\begin{gathered}
\int_{\mathcal{E}}\left|F_{n}-\chi_{A}\right| d \mu>\frac{1}{2} \mu(\mathcal{E}) \\
\int_{\mathcal{F}}\left|F_{n}-\chi_{A}\right| d \mu>\frac{1}{2} \mu(\mathcal{F}) .
\end{gathered}
$$

Se n é tal que $\left\|F_{n}-\chi_{A}\right\|_{1}<\frac{\epsilon}{4}$ concluímos que:

$$
\begin{gathered}
\left\|G_{n}-\chi_{A}\right\|_{1}=\int_{\mathcal{E}}\left|G_{n}-\chi_{A}\right| d \mu+\int_{\mathcal{F}}\left|G_{n}-\chi_{A}\right| d \mu+\int_{(\mathcal{E} \cup \mathcal{F})^{c}}\left|G_{n}-\chi_{A}\right| d \mu \\
\leq \mu(\mathcal{E})+\mu(\mathcal{F}) \leq 2 \frac{\epsilon}{4}+2 \frac{\epsilon}{4}=\epsilon .
\end{gathered}
$$

Usamos acima que $\left|G_{n}-\chi_{A}\right| \leq 1$ e que, em $(\mathcal{E} \cup \mathcal{F})^{c}$, as funções $G_{n}$ e $\chi_{A}$ coincidem. Também foram utilizadas as relações 4.1 e 4.2. Assim concluímos que $G_{n}$, de fato, converge a $\chi_{A}$ na norma $L^{1}$

Agora vamos provar o seguinte resultado que será usado no estudo da referência [7]:

Proposição 4.1.19: Seja $A \in \Sigma=\mathcal{B}\left(X_{t}, t \in[-\beta / 2, \beta / 2]\right.$ ) então $R \chi_{A}$ (se o processo for simétrico) e $U(t) \chi_{A}$ (se o processo for estacionário) são as funções características de algum conjunto em $\Sigma$ (ou melhor, estão na mesma classe de equivalência de uma função característica).

Prova: A prova é igual para $R$ ou $U(t)$. Vamos chamar estes operadores de $T$. Vimos no Teorema anterior que existem funções $G_{n}=H_{n}\left(X_{t_{1}}, \ldots, X_{t_{m(n)}}\right)$ que tendem a $\chi_{A}\left(\mathrm{em} L^{1}\right) \mathrm{e}$ assumem apenas valores 0 e 1 . Assim notamos que $G_{n}$ são funções características. As funções $T G_{n}$ também serão funções características como se pode ver abaixo:

$$
T G_{n}=H_{n}\left(X_{t_{1}^{*}}, \ldots, X_{t_{m(n)}^{*}}\right)
$$

em que $t_{j}^{*}$ é $t_{j}+t$ para $T=U(t)$ e $-t_{j}$ para $T=R$. Assim vemos que $T G_{n}$ assume apenas valores 0 ou 1 tal qual $H_{n}$. Como $T$ é uma isometria em $L^{1}$ temos que $T \chi_{A}=\lim _{n \rightarrow \infty} T G_{n}$, no limite em $L^{1}$. Agora basta notar que uma seqüência de funções características converge também a uma função que assume valores 0 ou 1 em quase toda parte; e, portanto, $T \chi_{A}$ é uma função característica (ou melhor, está na mesma classe de equivalência de uma). 
A última afirmação acerca da convergência de funções características pode ser vista da seguinte forma. Suponha que $K_{n}$ converge em $L^{1}$ para $K$ e todo $K_{n}$ é uma função característica. Seja o conjunto $A_{n}=K(x) \in\left[-\frac{1}{n}, \frac{1}{n}\right]^{c} \cup\left[1-\frac{1}{n}, 1+\frac{1}{n}\right]^{c}$. Então

$$
\int\left|K-K_{m}\right| d \mu=\int_{A_{n}}\left|K-K_{m}\right| d \mu+\int_{A_{n}^{c}}\left|K-K_{m}\right| d \mu \geq \frac{1}{n} \mu\left(A_{n}\right) .
$$

Como o limite do lado esquerdo é 0 , concluímos que $\mu\left(A_{n}\right)=0$. Logo $\mu\left(K(x)^{-1}\{0,1\}^{c}\right)=$ $\mu\left(\cup_{n=1}^{\infty} A_{n}\right) \leq \sum_{n} \mu\left(A_{n}\right)=0$. Assim $K$ assume valores 0 ou 1 em quase toda parte.

Agora vamos provar que além de isometrias os operadores $U(t)$ e $R$ satisfazem as relações $T(F G)=[T F][T G]$ se $F$ e $G$ pertencem a $L^{\infty}(Q, \Sigma, \mu)$ (em que $T$ é $R$ ou $U(t)$ ).

TEOREMA 4.1.20: Seja $F$ e $G$ pertencentes a $L^{\infty}$, então valem as relações $R(F G)=$ $[R F][R G]$ se o processo é simétrico e $U(t)(F G)=[U(t) F][U(t) G]$ se o processo é estacionário. Note que estamos pensando em $R$ e $U(t)$ definidos em $L^{p}(Q, \Sigma, \mu)$ para $p \in[1, \infty$ [ (tanto faz o p já que restritos a $L^{\infty}$ as aplicações $U(t)$ e $R$ são todas iguais, independente de $\left.p\right)$.

Prova:

Para provar isto, basta notar que se $F_{n} \rightarrow F$ então $F_{n} G \rightarrow F G$ independente da norma $L^{p}$ que se tome já que $G$ é essencialmente limitado. Da mesma forma notamos que $F_{n} G_{m} \rightarrow F_{n} G$ quando $m$ vai a infinito, desde que $F_{n}$ seja essencialmente limitado.

Assim seja $F_{n}\left(X_{t_{1}}, \ldots, X_{t_{m(n)}}\right)$ e $G_{n}\left(X_{t_{1}}, \ldots, X_{t_{q(n)}}\right)$ tais que $F_{n} \rightarrow F$ e $G_{n} \rightarrow G$ tal qual o Teorema 4.1.15. Então seja $T$ um dos operadores $R$ ou $U(t)$, então

$$
T(F G)=T\left(\lim _{n} F_{n} G\right)=\lim _{n} T\left(F_{n} G\right)=\lim _{n} T\left(F_{n} \lim _{k} G_{k}\right)=\lim _{n} \lim _{k} T\left(F_{n} G_{k}\right)=
$$

$\lim _{n} \lim _{k} T\left(F_{n}\left(X_{t_{1}}, \ldots, X_{t_{m(n)}}\right) G_{k}\left(X_{t_{1}}, \ldots, X_{t_{q(k)}}\right)\right)=\lim _{n} \lim _{k} F_{n}\left(X_{t_{1}^{*}}, \ldots, X_{t_{m(n)}^{*}}\right) G_{k}\left(X_{t_{1}^{*}}, \ldots, X_{t_{q(k)}^{*}}\right)=$

$$
\lim _{n} \lim _{k} T\left(F_{n}\left(X_{t_{1}}, \ldots, X_{t_{m(n)}}\right)\right) T\left(G_{k}\left(X_{t_{1}}, \ldots, X_{t_{q(k)}}\right)\right)=\lim _{n} T\left(F_{n}\right) \lim _{k} T\left(G_{k}\right)=T(F) T(G)
$$

em que $t_{j}^{*}$ é $t_{j}+t$ se o processo é estacionário e $-t_{j}$ se o processo é simétrico. O limite está sendo pensado como em qualquer das normas $L^{p}$ com $p$ entre $[1, \infty[$.

Podemos agora estender o Teorema 4.1.17. para os espaços $L^{\infty}\left(\Omega, \mathcal{B}\left(X_{t}, t \in \mathbb{R}\right), \mu\right)$. O resultado pode ser visto no seguinte Teorema:

\section{TEOREMA 4.1.21:}

1. Se o processo é estacionário então $U(t)$ se estende a grupo uniparamétrico de automor- 
fismos de $L^{\infty}\left(\Omega, \mathcal{B}\left(X_{t}, t \in \mathbb{R}\right), \mu\right)$.

2. Se o processo é simétrico então $R$ se estende a um automorfismo de $L^{\infty}\left(\Omega, \mathcal{B}\left(X_{t}, t \in\right.\right.$ $\mathbb{R}), \mu)$.

Prova:

A prova é a mesma para os operadores $R$ e $U(t)$. Vamos denotá-los genericamente de $T$. Dado $F \in L^{\infty}(Q, \Sigma, \mu)$ então como $\mu$ é uma medida de probabilidade, temos que $F \in$ $L^{1}(Q, \Sigma, \mu)$. Vamos definir o operador $T: L^{\infty}(Q, \Sigma, \mu) \rightarrow L^{\infty}(Q, \Sigma, \mu)$ como sendo a restrição do operador $T$, pensado como a isometria $T: L^{1}(Q, \Sigma, \mu) \rightarrow L^{1}(Q, \Sigma, \mu)$ do Teorema 4.1 .17 ao espaço $L^{\infty}(Q, \Sigma, \mu)$. Devemos notar que $T$ definido assim em $L^{\infty}(Q, \Sigma, \mu)$ é bem definido, pois se $F=0$ em relação a norma $L^{\infty}$, então $F=0$ em relação a norma $L^{1}$, e, portanto, $T F=0 \mathrm{em}$ relação a norma $L^{1}$ e também em relação a $L^{\infty}$. Evidentemente este operador é linear. Agora basta notar que ele é limitado. Vejamos a prova abaixo.

Dado $F \in L^{\infty}(Q, \Sigma, \mu)$, então existe uma seqüência de funções $F_{n}$ da forma $F_{n}=\sum_{k} a_{k}^{(n)} \chi_{A_{k}^{(n)}}$, em que $A_{k}^{(n)}$ são disjuntos, tais que $\left\|F_{n}\right\|_{\infty} \leq\|F\|_{\infty}$ e $F_{n} \rightarrow F$ tanto nas normas $L^{\infty}$ quanto na norma $L^{1}$. Como a aplicação de $T$ coincide com a isometria do Teorema 4.1 .17 e esta é contínua temos que:

$$
T F=T \lim _{n} F_{n}=\lim _{n} T F_{n}=\lim _{n} \sum_{k} a_{k}^{(n)} T \chi_{A_{k}^{(n)}}
$$

em que o limite é na norma $L^{1}$. Notamos agora que $T \chi_{A_{k}^{(n)}}$ são funções características pela proposição 4.1 .19 e são funções características de conjuntos disjuntos (a não ser por um conjunto de medida nula), pois se $k \neq l$ então $0=\left\|\chi_{A_{l}^{(n)}} \chi_{A_{k}^{(n)}}\right\|_{1}=\left\|T\left(\chi_{A_{l}^{(n)}} \chi_{A_{k}^{(n)}}\right)\right\|_{1}$ que, como vimos, é o mesmo de ||$T\left(\chi_{A_{l}^{(n)}}\right) T\left(\chi_{A_{k}^{(n)}}\right) \|_{1}$, logo $\left\|T F_{n}\right\|_{\infty} \leq \max _{k}\left|a_{k}^{(n)}\right| \leq\|F\|_{\infty}$. Logo como $T F_{n}$ tende a $T F$ na norma $L^{1}$, concluímos que $\|T F\|_{\infty} \leq\|F\|_{\infty}$. Assim $T$ é contínuo na norma $L^{\infty}$ e é um automorfismo, pelo Teorema 4.1.20.

Note que usamos acima que se funções $f_{n}$ são tais que $\left\|f_{n}\right\|_{\infty} \leq M$ e $f_{n} \rightarrow f$ na norma $L^{1}$, então $\|f\|_{\infty} \leq M$. A prova deste fato pode ser obtida definindo $A_{n}=\left\{x \in Q ;|f(x)|>M+\frac{1}{n}\right\}$. Assim

$$
\int\left|f-f_{m}\right| d \mu \geq \frac{1}{n} \mu\left(A_{n}\right)
$$

Tomando o limite no lado esquerdo, concluímos que $\mu\left(A_{n}\right)=0$. Logo $\mu(\{x \in Q ;|f(x)|>$ $M\})=\mu\left(\cup_{n=1}^{\infty} A_{n}\right)=0$. Assim $\|f\|_{\infty} \leq M$.

Vamos agora provar mais dois resultados que serão usados no estudo da referência [7]. Basicamente estamos interessados em conhecer um conjunto de funções densas em $L^{p}\left(Q, \mathcal{B}\left(X_{t}, t \in\right.\right.$ $[-\beta / 2, \beta / 2]), \mu)$, formado por combinações lineares de funções características.

ProposiçÃo 4.1.22: Seja $\left(\Omega_{i}, \mathcal{A}_{i}, \mu_{i}\right)_{i=1, \ldots, n}$ espaços de probabilidade. Então combinações 
lineares de funções do tipo $\chi_{A_{1}}\left(x_{1}\right) \ldots \chi_{A_{n}}\left(x_{n}\right)$ com $A_{1} \in \mathcal{A}_{1}, \ldots, A_{n} \in \mathcal{A}_{n}$ são densas em $L^{p}\left(\Omega_{1} \times\right.$ $\left.\ldots \times \Omega_{n}, \mathcal{A}_{1} \otimes \ldots \otimes \mathcal{A}_{n}, \mu_{1} \times \ldots \times \mu_{n}\right)$ para $p \in[1, \infty[$.

Prova: Sabemos que $\mathcal{A}_{1} \otimes \ldots \otimes \mathcal{A}_{n}$ é a $\sigma$-álgebra gerada por conjuntos do tipo $A_{1} \times \ldots \times A_{n}$ com $A_{1} \times \ldots \times A_{n} \in \mathcal{A}_{1} \times \ldots \times \mathcal{A}_{n}$ o que implica que $\mathcal{A}_{1} \otimes \ldots \otimes \mathcal{A}_{n}$ é a $\sigma$-álgebra $\left[\mathcal{A}_{1} \times \ldots \times \mathcal{A}_{n}\right]$. Porém sabemos que $\mathcal{A}_{1} \times \ldots \times \mathcal{A}_{n}$ é um semi-anel. Sabemos que anel[ $\left.\mathcal{A}_{1} \times \ldots \times \mathcal{A}_{n}\right]=\left\{A_{i} ; A\right.$ é a união finita disjunta de elementos de $\left.\mathcal{A}_{1} \times \ldots \times \mathcal{A}_{n}\right\}$ (lema 5.1.21, [31]). Também sabe-se que $\sigma$-anel $\left[\mathcal{A}_{1} \times \ldots \times \mathcal{A}_{n}\right]=\sigma$-anel $\left[\operatorname{anel}\left[\mathcal{A}_{1} \times \ldots \times \mathcal{A}_{n}\right]\right]$.

Pelo lema 4.1.14 temos que funções do tipo $h=\sum_{i=1}^{n} a_{i} \chi_{B_{i}}$, em que $B_{i} \in$ álgebra $\left[\mathcal{A}_{1} \times\right.$ $\left.\ldots \times \mathcal{A}_{n}\right]\left(=\right.$ anel $\left.\left[\mathcal{A}_{1} \times \ldots \times \mathcal{A}_{n}\right]\right)$ são densas em $L^{p}\left(\Omega_{1} \times \ldots \times \Omega_{n}, \mathcal{A}_{1} \otimes \ldots \otimes \mathcal{A}_{n}, \mu_{1} \times \ldots \times \mu_{n}\right)$.

Mas $B_{i}=\cup_{j=1}^{m(i)} A_{1 i}^{j} \times \ldots \times A_{n i}^{j}$, tal que $\left(A_{1 i}^{j} \times \ldots \times A_{n i}^{j}\right) \cap\left(A_{1 i}^{k} \times \ldots \times A_{n i}^{k}\right)=\emptyset$ se $i \neq k$.

$\operatorname{Assim} \chi_{B_{i}}=\sum_{j=1}^{m(i)} \chi_{A_{1 i}^{j} \times \ldots \times A_{n i}^{j}}\left(x_{1}, \ldots, x_{n}\right)=\sum_{j=1}^{m(i)} \chi_{A_{1 i}^{j}}\left(x_{1}\right) \ldots \chi_{A_{n i}^{j}}\left(x_{n}\right)$.

$\operatorname{Logo} h=\sum_{i=1}^{n} a_{i} \sum_{j=1}^{m(i)} \chi_{A_{1 i}^{j}}\left(x_{1}\right) \ldots \chi_{A_{n i}^{j}}\left(x_{n}\right)=\sum_{i=1}^{n} \sum_{j=1}^{m(i)} a_{i} \chi_{A_{1 i}^{j}}\left(x_{1}\right) \ldots \chi_{A_{n i}^{j}}\left(x_{n}\right)$.

CoroláRIo 4.1.23: O mesmo resultado vale para um espaço de probabilidade do tipo $\left(\Omega_{1} \times\right.$ $\left.\ldots \times \Omega_{n}, \mathcal{A}_{1} \otimes \ldots \otimes \mathcal{A}_{n}, \mu\right)$, ou seja, combinações lineares de funções do tipo $\chi_{A_{1}}\left(x_{1}\right) \ldots \chi_{A_{n}}\left(x_{n}\right)$ com $A_{1} \in \mathcal{A}_{1}, \ldots, A_{n} \in \mathcal{A}_{n}$ são densas em $L^{p}\left(\Omega_{1} \times \ldots \times \Omega_{n}, \mathcal{A}_{1} \otimes \ldots \otimes \mathcal{A}_{n}, \mu\right)$ para $p \in[1, \infty[$, se $\mu$ é uma medida de probabilidade.

Prova: a mesma dada acima.

Usando o Teorema acima, podemos provar o resultado desejado e já anunciado sobre funções densas em $L^{p}\left(Q, \mathcal{B}\left(X_{t}, t \in[-\beta / 2, \beta / 2]\right), \mu\right)$. Este resultado, como já foi dito, que nos será útil quando estudarmos o trabalho de Gérard e Jäkel [7].

TEOREMA 4.1.24: Seja $\left\{X_{t}\right\}_{t \in[-\beta / 2, \beta / 2]}$ um processo estocástico de $\left(Q, \mathcal{B}\left(X_{t}, t \in[-\beta / 2, \beta / 2]\right), \mu\right)$ em $\mathbb{R}$. Então combinações lineares de funções do tipo $\chi_{A_{1}}\left(x_{1}\right) \ldots \chi_{A_{n}}\left(x_{n}\right) \operatorname{com} A_{1} \in \mathcal{B}\left(X_{t_{1}}\right), \ldots, A_{n} \in$ $\mathcal{B}\left(X_{t_{n}}\right)$ são densas em $L^{p}\left(Q, \mathcal{B}\left(X_{t}, t \in[-\beta / 2, \beta / 2]\right), \mu\right)$ para $p \in[1, \infty[$.

Prova: Dado $F \in L^{p}\left(Q, \mathcal{B}\left(X_{t}, t \in[-\beta / 2, \beta / 2]\right), \mu\right)$. Então pelo Teorema 4.1 .15 existe uma função da forma $G\left(X_{t_{1}}, \ldots, X_{t_{n}}\right)$ tal que $G$ é mensurável e limitada e $\|F-G\|_{p}<\frac{\epsilon}{2}$.

Para continuar a prova, vamos construir a seguinte medida de probabilidade em $\mathbb{R}^{n}: A \ni$ $\mathbb{R}^{n} \rightarrow \mu^{\prime}(A)=\mu\left(\left(X_{t_{1}}, \ldots, X_{t_{n}}\right)^{-1}(A)\right)$. Então, pelo corolário anterior, temos uma função do tipo $H=\sum_{m} a_{m} \chi_{A_{1}^{m}}\left(x_{1}\right) \ldots \chi_{A_{n}^{m}}\left(x_{n}\right)$, tal que $\left[\int|H-G|^{p} d \mu^{\prime}\right]^{\frac{1}{p}}<\frac{\epsilon}{2}$. Porém sabemos por resultados conhecidos em teoria da medida (exemplo [31]) que:

$$
\left[\int\left|H\left(x_{1}, \ldots, x_{n}\right)-F\left(x_{1}, \ldots, x_{n}\right)\right|^{p} d \mu^{\prime}\right]^{\frac{1}{p}}=\left[\int\left|H\left(X_{t_{1}}, \ldots, X_{t_{n}}\right)-F\left(X_{t_{1}}, \ldots, X_{t_{n}}\right)\right|^{p} d \mu\right]^{\frac{1}{p}}
$$

Assim $\|F-H\|_{p} \leq\|F-G\|_{p}+\|G-H\|_{p}<\epsilon$. Agora basta notarmos que $\chi_{A}\left(X_{t}\right)=\chi_{X_{t}^{-1}(A)}$ é a função característica de um conjunto em $\mathcal{B}\left(X_{t}\right)$

A continuação desta seção terá como objetivo relacionar os operadores $R$ e $U(t)$ definidos 
anteriormente com os operadores de probabilidade condicional. Para isto vamos antes recordar a definição de probabilidade condicional [2].

Seja $\mathcal{F}$ uma $\sigma$-álgebra, $\mathcal{B} \subset \mathcal{F}$ uma sub- $\sigma$-álgebra de $\mathcal{F}$ e $\mu$ uma medida em $\mathcal{F}$. Dado uma função integrável $f$, mensurável por $\mathcal{F}$, dizemos que a probabilidade condicional de $f$ em relação $\mathcal{B}$, denotada por $E_{\mathcal{B}} f$, é a única função (a não ser por um conjunto de medida nula) mensurável em $\mathcal{B}$ e tal que para qualquer $A \in \mathcal{B}$ temos:

$$
\int_{A} f d \mu=\int_{A} E_{\mathcal{B}} f d \mu .
$$

Denotaremos a probabilidade condicional de $f$ em relação a $\mathcal{B}\left(X_{t}, t \in V\right)$ por $E_{V} f$. O procedimento padrão usado para mostrar que $g=E_{V} f$ será primeiro mostrar que $g$ é mensurável em $\mathcal{B}\left(X_{t}, t \in V\right)$ e, segundo, mostrar que vale a relação acima para qualquer que seja $A \in$ $\mathcal{B}\left(X_{t}, t \in V\right)$. Dadas estas definições podemos estudar as relações existentes entre $E_{V}, U(t)$ e $R$. Antes porém vejamos algumas propriedades interessantes da probabilidade condicional vista como um operador $E_{\mathcal{B}}: L^{p}(\Omega, \mathcal{F}, \mu) \rightarrow L^{p}(\Omega, \mathcal{F}, \mu)$.

ProposiçÃo 4.1.25: Seja $(\Omega, \mathcal{F}, \mu)$ um espaço de probabilidade e $\mathcal{B} \subset \mathcal{F}$. Se $F \in$ $L^{p}(\Omega, \mathcal{F}, \mu) . \quad$ Então $F=E_{\mathcal{G}} F+F^{\prime}$ tal que $\int_{\Omega} F^{\prime} G d \mu=0$ para qualquer que seja $G \in \mathcal{B}$ limitado $(p \in[1, \infty])$.

Prova: Seja $F^{\prime}=F-E_{\mathcal{G}} F$. Então para qualquer $A \in \mathcal{G}$ temos $\int_{A} F^{\prime} d \mu=\int_{A}\left(F-E_{\mathcal{G}} F\right) d \mu=$ 0 . Desta forma seja $G_{n}$ simples tal que $\left|G_{n}\right| \leq|G|$ e $G_{n}(x) \rightarrow G(x)$ e $G_{n}=\sum_{n=1}^{N} a_{n} \chi_{A_{n}}$, $A_{n} \in \mathcal{G}$. Assim como $\int\left|F^{\prime} G\right| d \mu \leq\|G\|_{\infty}|| F^{\prime} \|_{1}<\infty$ temos que $\left|F^{\prime} G_{n}\right|=\left|F^{\prime}\right|\left|G_{n}\right| \leq\left|F^{\prime}\right||G|$ que é integrável e, portanto, $\int_{\Omega} F^{\prime} G d \mu=\lim _{n \rightarrow \infty} \int_{\Omega} F^{\prime} G_{n} d \mu=\lim _{n \rightarrow \infty} \sum_{k=1}^{N(n)} a_{k}^{(n)} \int_{\Omega} \chi_{A_{k}^{(n)}} F^{\prime} d \mu=0$, pelo Teorema da convergência dominada.

ProposiçÃo 4.1.26: Seja $(\Omega, \mathcal{F}, \mu)$ um espaço de probabilidade e $\mathcal{G} \subset \mathcal{F}$. Então $E_{\mathcal{G}}:$ $L^{2}(\Omega, \mathcal{F}, \mu) \rightarrow L^{2}(\Omega, \mathcal{F}, \mu)$ é um operador linear contínuo.

Prova: Seja $F \in L^{2}(\Omega, \mathcal{F}, \mu)$. Logo

$$
\begin{gathered}
\int_{\Omega} E_{\mathcal{G}} F^{*} E_{\mathcal{G}} F d \mu=\int_{\Omega}\left|E_{\mathcal{G}} F^{*}\right|\left|E_{\mathcal{G}} F\right| d \mu \leq \int_{\Omega}\left(E_{\mathcal{G}}\left|F^{*}\right|\right)\left(E_{\mathcal{G}}|F|\right) d \mu= \\
=\int_{\Omega}\left(E_{\mathcal{G}}|F|\right)^{2} d \mu \leq \int_{\Omega} E_{\mathcal{G}}|F|^{2} d \mu=\int_{\Omega}|F|^{2} d \mu .
\end{gathered}
$$

Assim $<E_{\mathcal{G}} F, E_{\mathcal{G}} F>\leq<F, F>$ (Vide [2] pag. 316 e 317 para as desigualdades usadas, ou seja, $\left|E_{\mathcal{G}} F\right| \leq E_{\mathcal{G}}|F|$ e $\left.\left(E_{\mathcal{G}}|F G|\right)^{2} \leq\left(E_{\mathcal{G}}|F|^{2}\right)\left(E_{\mathcal{G}}|G|^{2}\right)\right)$.

Como corolário das duas proposições anteriores temos:

Corolário 4.1.27: Se $F \in L^{2}(\Omega, \mathcal{F}, \mu)$ então $F=E_{\mathcal{G}} F+F^{\prime}$, com $\mathcal{G} \subset \mathcal{F}$ e $E_{\mathcal{G}} F$ e $F^{\prime}$ $\in L^{2}(\Omega, \mathcal{F}, \mu)$. F' satisfaz $\int F^{\prime} G d \mu=0$ para qualquer que seja $G \in L^{2}(\Omega, \mathcal{G}, \mu)$. 
Prova: Basta repetir o argumento anterior, notando que $\int\left|F^{\prime} G\right| d \mu \leq\left(\int\left|F^{\prime}\right| d \mu\right)^{\frac{1}{2}}\left(\int|G|^{2} d \mu\right)^{\frac{1}{2}}$ $<\infty \operatorname{logo}\left|F^{\prime} G_{n}\right|$ está dominado por $\left|F^{\prime} G\right|$

CorolÁRIo 4.1.28: O operador $E_{\mathcal{G}}: L^{2}(\Omega, \mathcal{F}, \mu) \rightarrow L^{2}(\Omega, \mathcal{F}, \mu)$ é o operador de projeção no subespaço fechado $L^{2}(\Omega, \mathcal{G}, \mu)$, em que $\mathcal{G} \subset \mathcal{F}$.

Prova: Primeiro notamos que como $L^{p}(\Omega, \mathcal{G}, \mu)$ é um espaço de Banach, então ele é um subespaço fechado de $L^{p}(\Omega, \mathcal{F}, \mu)$. Depois notemos que $F=E_{\mathcal{G}} F+F^{\prime}$, em que $E_{\mathcal{G}} F \in L^{2}(\Omega, \mathcal{G}, \mu)$ e $F^{\prime} \in L^{2}(\Omega, \mathcal{F}, \mu)$. Como $F^{\prime}$ é ortogonal a qualquer elemento em $L^{2}(\Omega, \mathcal{G}, \mu)$ pelo corolário anterior, concluímos que $E_{\mathcal{G}} F+F^{\prime}$ é a decomposição de $F$ em $L^{2}(\Omega, \mathcal{G}, \mu) \oplus L^{2}(\Omega, \mathcal{G}, \mu)^{c}$. Como esta decomposição é única, concluímos que $E_{\mathcal{G}} F$ é a projeção de $F$ em $L^{2}(\Omega, \mathcal{G}, \mu)$.

Por fim, vamos ver um outro critério menos restritivo para saber se uma função é a esperança condicional em relação a uma sub- $\sigma$-álgebra.

LEMA 4.1.29: Seja $(\Omega, \mathcal{F}, \mu)$ um espaço de probabilidade. Seja $\mathcal{G} \subset \mathcal{F}$ uma $\sigma$-álgebra contido em $\mathcal{F}$. Se $\mathcal{R}$ é uma álgebra tal que $\mathcal{G}=\sigma$-álgebra $[\mathcal{R}$ ] e $F$ é integrável, então se $H \in \mathcal{G}$ (ou seja, $H$ é mensurável por $\mathcal{G}$ ) e $\int_{\Lambda} H d \mu=\int_{\Lambda} F d \mu$ para qualquer que seja $\Lambda \in \mathcal{R}$ temos que $H=E_{\mathcal{G}} F$.

Prova: Como $H \in \mathcal{G}$, basta provar que $\int_{\Lambda} H d \mu=\int_{\Lambda} F d \mu$ para qualquer que seja $\Lambda \in \mathcal{G}$. Seja $\mathcal{P}=\left\{\Lambda \in \mathcal{F} ; \int_{\Lambda} H d \mu=\int_{\Lambda} F d \mu\right\}$. Então $\mathcal{P} \ni \phi$ e $\mathcal{P}$ é uma classe monotônica, pois se $A_{n} \in \mathcal{P}$ e $A_{n} \uparrow A$ ou $A_{n} \downarrow A$ temos

$$
\int_{A} H d \mu=\lim _{n \rightarrow \infty} \int_{A_{n}} H d \mu=\lim _{n \rightarrow \infty} \int_{A_{n}} F d \mu=\int_{A} F d \mu
$$

pois $F$ e $H$ são integráveis, portanto $\left|\chi_{A_{n}} H\right| \leq|H|$ e podemos usar o Teorema da convergência dominada. Assim $\mathcal{P} \supset \sigma$-álgebra $[\mathcal{R}]$ e, portanto, $\mathcal{P} \supset \mathcal{G}$ pelo lema da classe monotônica. Assim, para qualquer $\Lambda \in \mathcal{G}$, vale que $\int_{\Lambda} H d \mu=\int_{\Lambda} F d \mu$.

Com estes resultados preliminares podemos, enfim, estudar as relações entre os diversos operadores.

TeORema 4.1.30: Se o processo é simétrico, então valem as relações:

$$
R^{2}=I, \quad R E_{t}=E_{-t} R, \quad R E_{0}=E_{0} R=E_{0}
$$

para qualquer que seja $t \in \mathbb{R}$. Se o processo é, além de simétrico, estacionário, então vale $R U(t)=U(-t) R$.

Prova: Vamos provar primeiro que $R^{2}=I$ e $R U(t)=U(-t) R$.

Dado $F\left(X_{t_{1}}, \ldots, X_{t_{n}}\right)$ então $R^{2} F\left(X_{t_{1}}, \ldots, X_{t_{n}}\right)=R F\left(X_{-t_{1}}, \ldots, X_{-t_{n}}\right)=F\left(X_{t_{1}}, \ldots, X_{t_{n}}\right)$ e

$R U(t) F\left(X_{t_{1}}, \ldots, X_{t_{n}}\right)=R F\left(X_{t_{1}+t}, \ldots, X_{t_{n}+t}\right)=F\left(X_{-t_{1}-t}, \ldots, X_{-t_{n}-t}\right)$

$U(-t) R F\left(X_{t_{1}}, \ldots, X_{t_{n}}\right)=U(-t) F\left(X_{-t_{1}}, \ldots, X_{-t_{n}}\right)=F\left(X_{-t_{1}-t}, \ldots, X_{-t_{n}-t}\right)$.

Logo $R^{2}=I$ e $R U(t)=U(-t) R$ coincidem nas funções da forma $F\left(X_{t_{1}}, \ldots, X_{t_{n}}\right)$. Como 
elas são densas para $L^{p}$ com $p \in[1, \infty$ [, de acordo com o Teorema 4.1.15, então isto basta para mostrar a igualdade entre os operadores. Como a atuação de $U(t)$ e $R$ agindo em $L^{\infty}$ é igual a restrição destes operadores agindo em $L^{1}$ (proposição 4.1.21), concluímos que o Teorema vale também para $L^{\infty}$.

Vamos provar agora que $R E_{t}=E_{-t} R$. Para isto vejamos que

1) $R E_{t} F=R \varphi\left(X_{t}\right)=\varphi\left(X_{-t}\right) \in \mathcal{B}\left(X_{-t}\right)$, em que $\varphi:(\mathbb{R}, \mathcal{B}(\mathbb{R})) \rightarrow(\mathbb{R}, \mathcal{B}(\mathbb{R}))$ é mensurável e $E_{t} F=\varphi\left(X_{t}\right)$.

2)Dado $\Lambda \in \mathcal{B}\left(X_{-t}\right) \log 0 \Lambda=X_{-t}^{-1}(A)$ para $A \in \mathcal{B}(\mathbb{R})$ e $F \in \mathcal{B}\left(X_{t}, t \in \mathbb{R}\right)$

$$
\begin{gathered}
\int_{\Lambda} R E_{t} F d \mu=\int_{\Omega} \chi_{A}\left(X_{-t}\right) R E_{t} F d \mu=\int_{\Omega} R\left(\chi_{A}\left(X_{t}\right) E_{t} F\right) d \mu=\int_{\Omega} E_{t}\left(\chi_{A}\left(X_{t}\right) F\right) d \mu \\
=\int_{\Omega} \chi_{A}\left(X_{t}\right) F d \mu=\int_{\Omega} R\left(\chi_{A}\left(X_{t}\right) F\right) d \mu=\int_{\Omega} \chi_{A}\left(X_{-t}\right)(R F) d \mu=\int_{X_{-t}^{-1}(A)} R F d \mu=\int_{\Lambda} R F d \mu .
\end{gathered}
$$

Assim temos que $R E_{t}=E_{-t} R$. Agora notemos que para $t=0$ temos:

1) $R E_{0} F=R \varphi\left(X_{0}\right)=\varphi\left(X_{0}\right) \in \mathcal{B}\left(X_{0}\right)$, em que $\varphi:(\mathbb{R}, \mathcal{B}(\mathbb{R})) \rightarrow(\mathbb{R}, \mathcal{B}(\mathbb{R}))$ é mensurável e $E_{0} F=\varphi\left(X_{0}\right)$.

2)Dado $\Lambda \in \mathcal{B}\left(X_{0}\right)$ temos que $\Lambda=X_{0}^{-1}(A)$ e, portanto,

$$
\begin{gathered}
\int_{\Lambda} R E_{0} F d \mu=\int_{\Omega} \chi_{A}\left(X_{0}\right) R E_{0} F d \mu=\int_{\Omega} R \chi_{A}\left(X_{0}\right) E_{0} F d \mu=\int_{\Omega} \chi_{A}\left(X_{0}\right) E_{0} F d \mu \\
=\int_{\Omega} E_{0} \chi_{A}\left(X_{0}\right) F d \mu=\int_{\Omega} \chi_{A}\left(X_{0}\right) F d \mu=\int_{X_{0}^{-1}(A)} F d \mu=\int_{\Lambda} F d \mu .
\end{gathered}
$$

Assim temos $R E_{0} F=E_{0} F \log 0 R E_{0}=E_{0}$

Agora vamos adicionar mais duas definições interessantes de processo estocástico: processos periódicos e processos OS-positivos.

DeFinição 4.1.31: Dizemos que um processo estocástico é periódico de período $\beta$ se $X_{t+\beta}=$ $X_{\beta}$ para todo $t \in \mathbb{R}$. Neste caso podemos indexá-lo por $S_{\beta}=\mathbb{R} / \beta \mathbb{Z}$. Se $\pi: \mathbb{R} \rightarrow S_{\beta}$ é a aplicação quociente temos que, dado $X_{t}$ com $t \in \mathbb{R}, X_{t}=X_{\pi(t)}$.

DeFINiÇÃo 4.1.32: Dizemos que um processo estocástico simétrico e periódico de período $\beta$ é OS-positivo em $S_{\beta}$ se

$$
\left\langle F^{*}\left(X_{-t_{1}}, \ldots, X_{-t_{n}}\right) F\left(X_{t_{1}}, \ldots, X_{t_{n}}\right)\right\rangle \geq 0
$$

sempre que $t_{1}, \ldots, t_{n} \in[0, \beta / 2]$ e $F$ é uma função mensurável e limitada em $\mathbb{R}^{n}$ para qualquer $n$ 
Podemos, então, relacionar estes conceitos da seguinte forma:

TeOrema 4.1.33: Se o processo é simétrico e periódico com perído $\beta$, então o processo é $O S$-positivo em $S_{\beta}$ se, e somente se, $E_{[0, \beta / 2]} R E_{[0, \beta / 2]} \geq 0$ como um operador em $L^{2}\left(\Omega, \mathcal{B}\left(X_{t}, t \in\right.\right.$ $[-\beta / 2, \beta / 2]), \mu)$, isto é, $<(R F)^{*} F>\geq 0$ para qualquer que seja $F \in L^{2}\left(\Omega, \mathcal{B}\left(X_{t}, t \in[0, \beta / 2]\right), \mu\right)$.

Prova:

$(\Leftarrow)$ Seja $F\left(X_{t_{1}}, \ldots, X_{t_{n}}\right), t_{1}, . ., t_{n} \in[0, \beta / 2]$, para $F$ limitada e mensurável. Logo a função $F\left(X_{t_{1}}, \ldots, X_{t_{n}}\right) \in L^{2}\left(\Omega, \mathcal{B}\left(X_{t}, t \in[0, \beta / 2]\right), \mu\right)$ e

$$
\begin{gathered}
\left\langle E_{[0, \beta / 2]} R E_{[0, \beta / 2]} F, F\right\rangle \geq 0 \Rightarrow \int_{\Omega}\left(E_{[0, \beta / 2]} R E_{[0, \beta / 2]} F\right)^{*} F d \mu \geq 0 \\
\Rightarrow \int_{\Omega}\left(E_{[0, \beta / 2]} F\left(X_{-t_{1}}, \ldots, X_{-t_{n}}\right)\right)^{*} F\left(X_{t_{1}}, \ldots, X_{t_{n}}\right) d \mu \geq 0
\end{gathered}
$$

e, portanto,

$\int_{\Omega}\left[E_{[0, \beta / 2]} F\left(X_{-t_{1}}, \ldots, X_{-t_{n}}\right)^{*}\right] F\left(X_{t_{1}}, \ldots, X_{t_{n}}\right) d \mu=\int_{\Omega} E_{[0, \beta / 2]}\left(F\left(X_{-t_{1}}, \ldots X_{-t_{n}}\right)^{*} F\left(X_{t_{1}}, \ldots, X_{t_{n}}\right)\right) d \mu$

$$
=\int_{\Omega} F\left(X_{-t_{1}}, \ldots, X_{-t_{n}}\right)^{*} F\left(X_{t_{1}}, \ldots, X_{t_{n}}\right) d \mu=\left\langle F^{*}\left(X_{-t_{1}}, \ldots, X_{-t_{n}}\right) F\left(X_{t_{1}}, \ldots, X_{t_{n}}\right)\right\rangle \geq 0
$$

$(\Rightarrow)$ Seja $F \in L^{2}\left(\Omega, \mathcal{B}\left(X_{t}, t \in[-\beta / 2, \beta / 2]\right), \mu\right)$ então temos

$$
<E_{[0, \beta / 2]} R E_{[0, \beta / 2]} F, F>=\int_{\Omega}\left(E_{[0, \beta / 2]} R E_{[0, \beta / 2]} F^{*}\right) F d \mu .
$$

Mas $F=E_{[0, \beta / 2]} F+F^{\prime}$ tal que $<F^{\prime}, G>=0$ para qualquer que seja $G \in L^{2}\left(\Omega, \mathcal{B}\left(X_{t}, t \in\right.\right.$ $[0, \beta / 2]), \mu)$.

Logo

$<E_{[0, \beta / 2]} R E_{[0, \beta / 2]} F, F>=<E_{[0, \beta / 2]} R E_{[0, \beta / 2]} F, E_{[0, \beta / 2]} F+F^{\prime}>=<E_{[0, \beta / 2]} R E_{[0, \beta / 2]} F, E_{[0, \beta / 2]} F>$

$=\int_{\Omega} E_{[0, \beta / 2]}\left(R E_{[0, \beta / 2]} F^{*}\right) E_{[0, \beta / 2]} F d \mu=\int_{\Omega}\left(R E_{[0, \beta / 2]} F^{*}\right) E_{[0, \beta / 2]} F d \mu=<R E_{[0, \beta / 2]} F, E_{[0, \beta / 2]} F>$.

Porém sabemos que função do tipo $G=G\left(X_{t_{1}}, \ldots, X_{t_{n}}\right) t_{1}, \ldots t_{n} \in[0, \beta / 2]$ são densas em $L^{2}\left(\Omega, \mathcal{B}\left(X_{t}, t \in[0, \beta / 2]\right), \mu\right)$. Seja $G_{n} \rightarrow E_{[0, \beta / 2]} F \Rightarrow R G_{n} \rightarrow E_{[0, \beta / 2]} F$ (pois $R$ é contínuo). 
Assim $<R E_{[0, \beta / 2]} F, E_{[0, \beta / 2]} F>=\lim _{n \rightarrow \infty}<R G_{n}, G_{n}>\geq 0$. (Notemos que se $x_{n} \rightarrow x$ e $y_{n} \rightarrow y$ então $<x_{n}, y_{n}>-<x, y>=<x_{n}, y_{n}>-<x, y_{n}>+<x, y_{n}>-<x, y>$ que vai a zero).

\subsection{O trabalho de Klein e Landau}

O passo crucial para caracterizar estados quânticos em equilíbrio térmico usado neste trabalho foi dado em um artigo escrito em 1979 por Abel Klein e Lawrence Landau [15]. A idéia deste artigo é fazer uma correspondência biunívoca entre certos estados KMS e certos tipos de processos estocásticos. Para uma afirmação mais precisa é necessário que façamos certas definições.

O tipo de estado KMS utilizado neste artigo é o sistema KMS estocasticamente positivo. Para tratar estes estados precisamos de alguns Teoremas a fim de dar sentido às definições necessárias. Vamos, então, enunciar o Teorema de Araki sobre extensão analítica de certas funções de operadores.

TEOREMa 4.2.1 (ARAKI) [15]: Dados $\mathcal{B}$ uma álgebra $C^{*}$ e $\omega$ um estado fiel que satisfaz a condição KMS. Seja $\overline{\mathcal{B}}$ a álgebra de von Neumann gerada pela imagem da representação de $\mathcal{B}$ na representação $G N S$ de $\omega$. Definindo o conjunto $I_{\beta}(n)=\left\{\left(z_{1}, \ldots, z_{n}\right) \in\right.$ $\mathbb{C}^{n} ;$ Im $z_{j}>0$ paraj $\left.=1, \ldots, n \sum_{j=1}^{n} \operatorname{Im} z_{j}<\beta / 2\right\}$ temos que se $\left(z_{1}, \ldots, z_{n}\right) \in \bar{I}_{\beta}(n)$, então para todo $A_{1}, \ldots, A_{n} \in \overline{\mathcal{B}}$ vale que $A_{n} e^{i z_{n-1} H} A_{n-1} \ldots e^{i z_{1} H} A_{1} \Omega \in \mathcal{D}\left(e^{i z_{n} H}\right)$, e, como função de $\left(z_{1}, \ldots, z_{n}\right), e^{i z_{n} H} A_{n} e^{i z_{n-1} H} A_{n-1} \ldots e^{i z_{1} H} A_{1} \Omega$ é analítico em norma em $I_{\beta}(n)$, contínuo em em norma em $\bar{I}_{\beta}(n)$, e uniformemente limitado em $\bar{I}_{\beta}(n)$ por $\left\|A_{1}\right\|\left\|A_{2}\right\| \ldots\left\|A_{n}\right\|$. Além disto o vetor $\exp \left(-\left(\beta / 2+i \sum_{j=1}^{n} z_{j}\right) H\right) A_{n} e^{i z_{n-1} H} A_{n-1} \ldots e^{i z_{1} H} \Omega$ é analítico em norma em $I_{\beta}(n-1)$, $e$ continuo em norma em $\bar{I}_{\beta}(n-1)$.

Como conseqüência deste Teorema a função de correlação:

$$
\Gamma_{A_{1}, \ldots, A_{n}}\left(t_{1}, \ldots, t_{n}\right)=\left(\Omega, \bar{\alpha}_{t_{1}}\left(A_{1}\right) \ldots \bar{\alpha}_{t_{n}}\left(A_{n}\right) \Omega\right)
$$

pode ser analiticamente continuada para variáveis puramente imaginárias $z_{j}=i s_{j}$ em que $-\beta / 2 \leq s_{1} \leq \ldots \leq s_{k} \leq 0 \leq s_{k+1} \leq . . \leq s_{n} \leq \beta / 2$. Desta forma:

$$
\begin{gathered}
\Gamma_{A_{1}, \ldots, A_{n}}\left(i s_{1}, \ldots, i s_{n}\right)= \\
\left(e^{s_{k}} A_{k}^{*} e^{-\left(s_{k}-s_{k-1}\right) H} A_{k-1}^{*} \ldots e^{-\left(s_{2}-s_{1}\right) H} A_{1}^{*} \Omega, e^{-s_{k+1} H} A_{k+1} e^{-\left(s_{k+2}-s_{k+1}\right) H} A_{k+2} \ldots e^{-\left(s_{n}-s_{n-1}\right) H} A_{n} \Omega\right) .
\end{gathered}
$$

Desta forma se $A_{1}, \ldots, A_{n} \in \mathcal{B}$, então $\omega\left(\alpha_{t_{1}}\left(A_{1}\right) \ldots \alpha_{t_{n}}\left(A_{n}\right)\right)$ tem uma continuação analítica da forma dada pela fórmula anterior. Com estas informações, já é possível dar sentido a seguinte 
definição:

DEFINIÇÃO 4.2.2: O estado KMS é dito ser estocasticamente positivo com respeito a sub-álgebra $C^{*}, \mathcal{U}$ de $\mathcal{B}$ se, para todo $A_{1}, \ldots, A_{n} \in \mathcal{U}_{+}=\{A \in \mathcal{U} ; A \geq 0\}$, as funções $\Gamma_{A_{1}, \ldots, A_{n}}\left(i s_{1}, \ldots, i s_{n}\right)$ não são negativas.

Partindo desta definição já é possível especificar os "certos" estados KMS aos quais o artigo de Klein e Landau [15] se refere. Chamamos estes estados de sistemas KMS estocasticamente positivos. A definição é dada por:

DEFINIÇÃO 4.2.3 [15]: Um sistema KMS estocasticamente positivo com temperatura inversa $\beta$ é uma quádrupla $\left(\mathcal{B}, \mathcal{U}, \alpha_{t}, \omega\right)$ que consiste em

- Uma álgebra $C^{*} \mathcal{B}$.

- Um grupo uniparamétrico de automorfismos $\alpha_{t}$ agindo em $\mathcal{B}$.

- Uma sub-álgebra abeliana, $\mathcal{U}$, de $\mathcal{B}$ tal que a álgebra gerada por $\cup_{t \in \mathbb{R}} \alpha_{t}(\mathcal{U})$ é igual à $\mathcal{B}$.

- Um estado fiel em $\mathcal{B}$ satisfazendo a condição KMS na temperatura inversa $\beta$, relativo a $\alpha_{t}$.

Este estado deve ser estocasticamente positivo.

Uma vez que se especificou o estado KMS que se trabalha iremos agora especificar a quais processos estocásticos nos referimos.

Usaremos processos indexados por $T=\mathbb{R}$ com variáveis aleatórias $X_{t}:(\Omega, \Sigma, \mu) \rightarrow(K, \mathcal{B}(K))$ que são:

1) Simétricos.

2) Estacionários.

3) Fracamente estocasticamente contínuo: Dizemos que um processo é fracamente contínuo se dado $\eta$ e $\epsilon$, existe $\delta>0$ tal que se $\left|t-t_{0}\right|<\delta$ implica que $\mu\left(\left|f\left(X_{t}\right)-f\left(X_{0}\right)\right|>\eta\right)<\epsilon$, para qualquer $f:(K, \mathcal{B}(K)) \rightarrow(\mathbb{R}, \mathcal{B}(\mathbb{R}))$ limitada e mensurável, ou seja, que

$$
\lim _{t \rightarrow t_{0}} \mu\left(\left|f\left(X_{t}\right)-f\left(X_{t_{0}}\right)\right|>\eta\right)=0
$$

para qualquer $\eta>0$.

4) Fiel: Dizemos que o processo é fiel se $<f\left(X_{t}\right)>\neq 0$ para toda $f \in C_{\mathbb{R}}(K)$ não negativa e não nula, ou seja, toda função contínua em $K$ com valores reais com $f \geq 0$ e $f \neq 0$.

5) Periódico (de período $\beta$ ).

6) OS-positivo. 
Com estas definições estamos em posição de enunciar o Teorema de Klein Landau que será usado então:

TeOrema De Klein Landau 4.2.4 [15]: Seja $\left(\mathcal{B}, \mathcal{U}, \alpha_{t}, \omega\right)$ um sistema estocasticamente positivo KMS na temperatura inversa $\beta$. Então existe um processo estocástico $\left\{X_{t}\right\}_{t \in \mathbb{R}}$, com valores no espectro de $\mathcal{U}$, que é periódico com período $\beta$, estacionário, simétrico, fracamente estocasticamente continuo, fiel e OS-positivo tal que:

$$
<A_{1}\left(X_{t_{1}}\right) \ldots A_{n}\left(X_{t_{n}}\right)>=\Gamma_{A_{1}, \ldots, A_{n}}\left(i t_{1}, \ldots, i t_{n}\right)
$$

para todo $A_{1}, \ldots, A_{n} \in \mathcal{U}$ e $t_{1}, \ldots, t_{n} \in \mathbb{R}$ com $t_{1} \leq t_{2} \leq \ldots \leq t_{n}$ e $t_{n}-t_{1} \leq \beta$. Tal processo é único a não ser por equivalência.

Conversamente seja $\left\{X_{t}\right\}_{t \in \mathbb{R}}$ um processo estocástico com valores num espaço Hausdorff compacto $K$, periódico com período $\beta$, estacionário, simétrico, fracamente estocasticamente continuo, fiel e OS-positivo em $S_{\beta}$. Então existe um sistema estocasticamente positivo KMS $\left(\mathcal{B}, \mathcal{U}, \alpha_{t}, \omega\right)$ na temperatura inversa $\beta$ tal que $\mathcal{U} \cong C(K)$ e a relação acima vale. Um sistema KMS construído desta forma é único a não ser por isometria.

\subsection{O efeito Unruh do ponto de vista estocástico}

Nesta seção mostraremos uma nova forma de ver o efeito Unruh partindo do trabalho de Klein-Landau [15] e de Gérard-Jäkel [7]. Não pretende-se aqui provar todas as passagens, nem sabemos como fazê-lo. Queremos apenas esboçar uma estratégia, uma outra maneira de se ver o problema baseado no Teorema de Klein-Landau. O que se fará aqui é seguir passo a passo estes artigos e fazer as devidas alterações a fim de aplicá-lo ao problema do efeito Unruh.

Antes de mais nada, vamos primeiramente esboçar o procedimento que será seguido nesta seção. O que se fará é pegar as funções de Schwinger parametrizadas por $(r, \theta, y, z)$ tal qual o artigo de Fröhlich e Birke [12]. A seguir vamos definir o conceito de espaço de trajetória generalizado. Mostraremos, então, como o Teorema de Klein-Landau pode ser escrito em termos destes espaços de trajetória. Após isto, daremos um esquema que mostra, usando as funções de Schwinger e tomando o ângulo $\theta$ como o tempo imaginário, como obter um espaço de trajetória generalizado, que por sua vez implica na obtenção de um estado KMS (de acordo com o Teorema de Klein-Landau escrito para espaços de trajetórias generalizados, como será enunciado nas próximas seções).

Para entendermos melhor como isto explica o efeito Unruh, vamos mostrar como a rotação de ângulo $\theta$ se relaciona com o boost de Lorentz na direção $x$. Lembrando que o boost de Lorentz na direção $x$ é dado por: 


$$
\Lambda(s)=\left(\begin{array}{cccc}
\cosh (s) & \sinh (s) & 0 & 0 \\
\sinh (s) & \cosh (s) & 0 & 0 \\
0 & 0 & 1 & 0 \\
0 & 0 & 0 & 1
\end{array}\right)
$$

Podemos, então, fazer a extensão analítica de $\Lambda(s)$ para o grupo de Lorentz complexo e obter a transformação $\Lambda(i s)$ em que $s$ é um número real. Se aplicarmos esta transformação à $(i t, x, y, z)$, que são as coordenadas em que estão definidas as funções de Schwinger, e parametrizarmos $(t, x, y, z)$ usando coordenadas $(r, \theta, y, z)$ dadas por $t=i r \sin (\theta)$ e $x=r \cos (\theta)$ obtemos o seguinte resultado:

$$
\begin{gathered}
\Lambda(i s)\left(\begin{array}{c}
i t \\
x \\
y \\
z
\end{array}\right)=\Lambda(i s)\left(\begin{array}{c}
i r \sin (\theta) \\
r \cos (\theta) \\
y \\
z
\end{array}\right)=\left(\begin{array}{cccc}
\cos (s) & i \sin (s) & 0 & 0 \\
i \sin (s) & \cos (s) & 0 & 0 \\
0 & 0 & 1 & 0 \\
0 & 0 & 0 & 1
\end{array}\right)\left(\begin{array}{c}
i r \sin (\theta) \\
r \cos (\theta) \\
y \\
z
\end{array}\right) \\
=\left(\begin{array}{c}
i r \sin (\theta+s) \\
r \cos (\theta+s) \\
y \\
z
\end{array}\right) .
\end{gathered}
$$

Observamos, assim, que $\Lambda(i s)(r, \theta, y, z)=(r+s, \theta, y, z)$. Desta forma, concluímos que a rotação por um ângulo $s$ corresponde a ação do boost $\Lambda(i s)$. Porém notemos que a rotação por um ângulo $s$ corresponde, no nosso esquema, justamente ao tempo imaginário. Logo a translação de tempo real do estado KMS corresponde ao grupo de automorfismo do boost de Lorentz na direção $x$. Portanto, concluímos que o estado KMS (de equilíbrio) se refere aos referenciais uniformemente acelerados, obtendo assim uma nova versão do efeito Unruh.

Vamos agora esclarecer melhor o esquema e dar a definição de espaço de trajetória generalizado.

\subsubsection{Espaço de trajetória generalizado}

Para fazer a reconstrução do espaço de Hilbert físico vamos seguir a referência [7]. De acordo com este artigo, para fazê-la não é necessário um conhecimento detalhado das variáveis aleatórias $X_{t}$, tampouco do espaço topológico $K$ no qual as variáveis aleatórias tomam seus valores. Precisa-se apenas da estrutura de espaço de trajetórias generalizado. A definição será dada a seguir.

DEFINIÇÃO 4.3.1.1 [7]: Um espaço de trajetória generalizado $\left(Q, \Sigma, \Sigma_{0}, U(t), R, \mu\right)$ consiste de: 
(i) Um espaço de probabilidade $(Q, \Sigma, \mu)$.

(ii) Uma sub-álgebra distinta $\Sigma_{0}$.

(iii) Um grupo uniparamétrico $\mathbb{R} \ni t \rightarrow U(t)$ de ${ }^{*}$-automorfismos de $L^{\infty}(Q, \Sigma, \mu)$ que preservam a medida e que são fortemente contínuos em medida.

(iv) $U m^{*}$-automorfismo $R$ que preserva medidas em $L^{\infty}(Q, \Sigma, \mu)$ tal que $R U(t)=U(-t) R$, $R^{2}=I$ e $R E_{0}=E_{0} R$, em que $E_{0}$ é a esperança condicional com respeito a $\sigma$-álgebra $\Sigma_{0}$.

Além disto queremos que

(v) $\Sigma=\bigvee_{t \in \mathbb{R}} U(t) \Sigma_{0}$.

Entendemos um automorfismo $T$ que preserva medidas da seguinte forma: $T$ é tal que quando aplicado a uma função característica o resultado também é uma função característica, ou seja, dado um conjunto $A \in \Sigma$ e sua função característica $\chi_{A}$ então $T \chi_{A}$ também é uma função característica de um conjunto $B \in \Sigma$. Assim podemos entender $T$ também como uma função de $\Sigma$ em $\Sigma$, usando a relação biunívoca entre funções características e conjuntos. (Note, no entanto, que como função de $\Sigma$ em $\Sigma$ consideramos conjuntos que diferem por um subconjunto de medida nula iguais, logo mais precisamente estamos tratando de classes de equivalências). $\mathrm{O}$ fato de preservar medidas deve ser entendido da seguinte forma: seja $A \in \Sigma$ e $B$ o conjunto cuja função característica é $T \chi_{A}$, então $\mu(A)=\mu(B)$, ou seja $\mu(A)=\mu(T A)$.

A parte $(\mathrm{v})$ acima significa que a $\sigma$-álgebra $\Sigma$ é gerada pelos conjuntos $U(t) S$ para todo $t \in \mathbb{R}$ e $S \in \Sigma_{0}$. De (iii) e (iv) segue que $U(t)$ e $R$ se estendem a um grupo de isometria fortemente contínuo de $L^{p}(Q, \Sigma, \mu)$ para $1 \leq p<\infty$.

Em termos do espaço de trajetórias a periodicidade e a OS-positividade podem ser formuladas da seguinte maneira: O espaço de trajetória $\left(Q, \Sigma, \Sigma_{0}, U(t), R, \mu\right)$ é $\beta$-periódico se $U(\beta)=I$. Podemos então considerar $U(t)$ indexado pelo círculo $S_{\beta}=[-\beta / 2, \beta / 2]$. Ele é OS-positivo se $E_{[0, \beta / 2]} R E_{[0, \beta / 2]} \geq 0$ como um operador em $L^{2}(Q, \Sigma, \mu)$.

Neste momento é interessante e importante saber exatamente como a estrutura proposta por Klein e Landau [15] se relaciona com um espaço de trajetória generalizado. Para isto provaremos o seguinte Teorema que relacionará os processos estocásticos apresentados em [15] com a estrutura apresentada acima.

TEOREMa 4.3.1.2: Seja $(Q, \Sigma, \mu)$ um espaço de probabilidade e $\left\{X_{t}\right\}$ um processo estocástico com valores em $K$, um espaço compacto (com a $\sigma$-álgebra de Borel). Seja $\Sigma=\mathcal{B}\left(X_{t}, t \in\right.$ $[-\beta / 2, \beta / 2])$. Suponhamos que o processo é periódico de periodo $\beta$, estacionário, simétrico, fiel, fracamente estocasticamente positivo em $S_{\beta}$ e $O S$-positivo. Então $\left(Q, \Sigma, \Sigma_{0}, U(t), R, \mu\right)$ é um espaço de trajetória generalizado OS-positivo e períódico de periodo $\beta$, em que $\Sigma_{0}=\mathcal{B}\left(X_{0}\right)$, $U(t)$ e $R$ são os operadores definidos na seção 4.1.

Prova: Vamos analisar ítem por ítem da definição acima de espaço de trajetória generalizado. Os ítens (i) e (ii) são imediatos.

No ítem (iii) podemos observar que dado $A \in \Sigma$, temos $\mu(A)=\left\|\chi_{A}\right\|_{1}=\left\|U(t) \chi_{A}\right\|_{1}=$ 
$\mu\left(U(t) \chi_{A}\right)$, em que usamos o fato de que $\chi_{A} \in L^{1}(Q, \Sigma, \mu)$ e que $U(t)$ é uma isometria em $L^{1}(Q, \Sigma, \mu)$, tal como explicado no Teorema 4.1.17. Também usamos o fato de que $U(t) \chi_{A}$ é a função característica de algum conjunto, tal qual mostrado na proposição 4.1.19. O fato de ser um automorfismo de $L^{\infty}$ pode ser visto no Teorema 4.1.21. A continuidade forte em medida é mais complicada e pode ser vista da seguinte forma.

Primeiramente notamos que $\lim _{t \rightarrow t_{0}} \mu\left(\left|f\left(X_{t}\right)-f\left(X_{t_{0}}\right)\right|>\eta\right) \rightarrow 0$, para qualquer que seja $\eta>0$ (com $f$ limitada e mensurável), implica que $\lim _{t \rightarrow t_{0}}\left\|f\left(X_{t}\right)-f\left(X_{t_{0}}\right)\right\|_{1} \rightarrow 0$. Para provar isto basta escolhermos $\eta=\epsilon$ e assim obter:

$$
\begin{gathered}
\int_{Q}\left|f\left(X_{t}\right)-f\left(X_{t_{0}}\right)\right| d \mu=\int_{\left[\left|f\left(X_{t}\right)-f\left(X_{t_{0}}\right)\right|>\eta\right]}\left|f\left(X_{t}\right)-f\left(X_{t_{0}}\right)\right| d \mu+\int_{\left[\left|f\left(X_{t}\right)-f\left(X_{t_{0}}\right)\right|<\eta\right]}\left|f\left(X_{t}\right)-f\left(X_{t_{0}}\right)\right| d \mu \\
\leq 2 \mu\left(\left|f\left(X_{t}\right)-f\left(X_{t_{0}}\right)\right|>\eta\right)\|f\|_{\infty}+\epsilon .
\end{gathered}
$$

Logo $\lim \sup _{t \rightarrow t_{0}}\left\|f\left(X_{t}\right)-f\left(X_{t_{0}}\right)\right\|_{1}<\epsilon$ para qualquer $\epsilon$ e assim $\lim _{t \rightarrow t_{0}}\left\|f\left(X_{t}\right)-f\left(X_{t_{0}}\right)\right\|_{1} \rightarrow$ 0. Assim dado $A \in \mathcal{B}\left(X_{t_{0}}\right)$ então $A=X_{t_{0}}^{-1}(B) \operatorname{com} B \in K$ e $\chi_{A}=\chi_{X_{t_{0}}^{-1}(B)}=\chi_{B}\left(X_{t_{0}}\right) \mathrm{e}$ assim $\lim _{t \rightarrow 0}\left\|U(t) \chi_{A}-\chi_{A}\right\|_{1}=\lim _{t \rightarrow 0}\left\|U(t) \chi_{B}\left(X_{t_{0}}\right)-\chi_{B}\left(X_{t_{0}}\right)\right\|_{1}$ que pode ser escrito como $\lim _{t \rightarrow 0}\left\|\chi_{B}\left(X_{t+t_{0}}\right)-\chi_{B}\left(X_{t_{0}}\right)\right\|_{1}=0$. Agora notamos que se $A \in \mathcal{B}\left(X_{t_{0}}\right)$ e $C \in \mathcal{B}\left(X_{t_{1}}\right)$ então

$$
\left\|U(t)\left(\chi_{A} \chi_{C}\right)-\chi_{A} \chi_{C}\right\|_{1}=\left\|U(t)\left(\chi_{A} \chi_{C}\right)-\left(U(t) \chi_{A}\right) \chi_{C}+\left(U(t) \chi_{A}\right) \chi_{C}-\chi_{A} \chi_{C}\right\|_{1}=
$$

$\left\|\left(U(t) \chi_{A}\right)\left(U(t) \chi_{C}-\chi_{C}\right)\right\|_{1}+\left\|\left(U(t) \chi_{A}-\chi_{A}\right) \chi_{C}\right\|_{1} \leq\left\|U(t) \chi_{C}-\chi_{C}\right\|_{1}+\left\|U(t) \chi_{A}-\chi_{A}\right\|_{1} \rightarrow 0$

em que usamos que $\left|U(t) \chi_{A}\right| \leq 1$. Agora notamos que seguindo o raciocínio, basta tormarmos

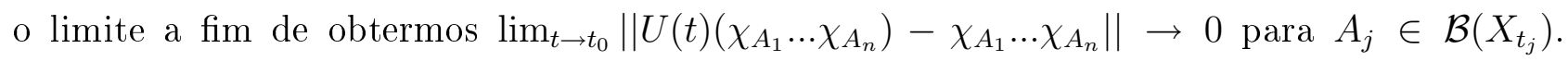

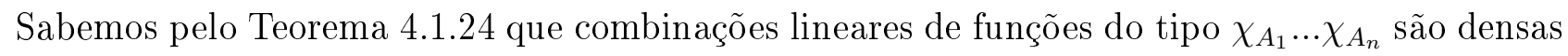
em $L^{1}(Q, \Sigma, \mu)$. Assim dada $F \in L^{\infty}(Q, \Sigma, \mu)$, seja $\sum_{m=1}^{M} a_{m} \chi_{A_{1}^{m}} \ldots \chi_{A_{n}^{m}}$ em que $A_{j}^{m} \in \mathcal{B}\left(X_{t_{j}}\right)$ para $j$ entre 1 e $n$ tal que $\left\|F-\sum_{m=1}^{M} a_{m} \chi_{A_{1}^{m} \ldots} \chi_{A_{n}^{m}}\right\|_{1}<\frac{\epsilon}{2}$. Concluímos então que $\|U(t) F-F\|_{1}$ é igual à

$$
\begin{gathered}
\| U(t) F-U(t) \sum_{m=1}^{M} a_{m} \chi_{A_{1}^{m}} \ldots \chi_{A_{n}^{m}}+U(t) \sum_{m=1}^{M} a_{m} \chi_{A_{1}^{m}} \ldots \chi_{A_{n}^{m}}- \\
-\sum_{m=1}^{M} a_{m} \chi_{A_{1}^{m} \ldots \chi_{A_{n}^{m}}}+\sum_{m=1}^{M} a_{m} \chi_{A_{1}^{m}} \ldots \chi_{A_{n}^{m}}-F \|_{1} \leq
\end{gathered}
$$




$$
\begin{aligned}
& \left\|U(t) F-U(t) \sum_{m=1}^{M} a_{m} \chi_{A_{1}^{m}} \ldots \chi_{A_{n}^{m}}\right\|_{1}+\left\|U(t) \sum_{m=1}^{M} a_{m} \chi_{A_{1}^{m}} \ldots \chi_{A_{n}^{m}}-\sum_{m=1}^{M} a_{m} \chi_{A_{1}^{m} \ldots \chi_{A_{n}^{m}}}\right\|_{1}+
\end{aligned}
$$

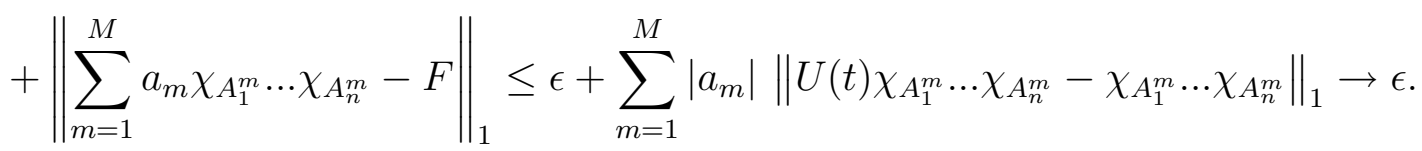

Logo, $\limsup _{t \rightarrow 0}\|U(t) F-F\|_{1}<\epsilon$ para qualquer que seja $\epsilon$. Portanto $\lim _{t \rightarrow 0} \| U(t) F-$ $F \|_{1}=0$. Concluímos que $U(t)$ é fortemente contínuo na norma $L^{1}$ (para $F \in L^{\infty}(Q, \Sigma, \mu)$ ) e, como conseqüência, em medida. Esta última afirmação pode ser provada da seguinte forma.

Se $U(t)$ não é fortemente contínua em medida em 0 , existe $F \in L^{\infty}(Q, \Sigma, \mu), \eta_{0}$ e $\epsilon_{0}$ maiores do que zero tal que para qualquer $\delta>0$ existe $t^{\prime}$ satisfazendo $\left|t^{\prime}-t_{0}\right|<\delta$ que satisfaz $\mu(\mid U(t) F-$ $\left.F \mid>\eta_{0}\right)>\epsilon_{0}$. Isto implica que

$$
\int_{Q}|U(t) F-F| d \mu \geq \int_{\left[|U(t) F-F|>\eta_{0}\right]}|U(t) F-F| d \mu \geq \eta_{0} \mu\left(|U(t) F-F|>\eta_{0}\right) \geq \eta_{0} \epsilon_{0} .
$$

Desta forma $\|U(t) F-F\|_{1} \nrightarrow 0$ o que é uma contradição.

No ítem (iv), as relações $R U(t)=U(-t) R, R^{2}=I$ e $R E_{0}=E_{0} R$ podem ser vistas diretamente como uma conseqüência do Teorema 4.1.30. O fato de preservar a medida pode ser vista da seguinte forma. Seja $A \in \Sigma$. Então $\mu(A)=\left\|\chi_{A}\right\|_{1}=\left\|R \chi_{A}\right\|_{1}=\mu\left(R \chi_{A}\right)$, em que usamos o fato de que $\chi_{A} \in L^{1}(Q, \Sigma, \mu)$ e que $R$ é uma isometria em $L^{1}(Q, \Sigma, \mu)$, tal como explicado no Teorema 4.1.17. Também utilizamos o fato de que $U(t) \chi_{A}$ é a função característica de algum conjunto tal qual mostrado na proposição 4.1.19. O fato de ser um automorfismo de $L^{\infty}$ pode ser visto no Teorema 4.1.21.

O ítem (v) pode ser visto da seguinte forma. Seja $A \in \mathcal{B}\left(X_{t}\right)$. Então $A=X_{t}^{-1}(B)$ em que $B \in K$. Assim dada a função característica de $A, \chi_{A}(x)$, podemos escrevê-la como $\chi_{A}(x)=\chi_{X_{t}^{-1}(B)}(x)=\chi_{B}\left(X_{t}(x)\right.$ ) (note que $\chi_{A}: Q \rightarrow \mathbb{R}$ e $\chi_{B}: K \rightarrow \mathbb{R}$ ). Concluímos assim que $\chi_{A}(x)=U(t) \chi_{B}\left(X_{0}(x)\right)$. Portanto $A \in \bigvee_{t \in \mathbb{R}} U(t) \Sigma_{0}$ e $\mathcal{B}\left(X_{t}\right) \subset \bigvee_{t \in \mathbb{R}} U(t) \Sigma_{0}$. Assim concluímos que toda função $X_{t}$ é mensurável por $\bigvee_{t \in \mathbb{R}} U(t) \Sigma_{0}$. Logo $\Sigma=\bigvee_{t \in \mathbb{R}} U(t) \Sigma_{0}$.

A propriedade de OS-periodicidade pode ser vista diretamente do Teorema 4.1.33.

A periodicidade pode ser vista como conseqüência da periodicidade do processo estocástico, pois

$$
U(\beta) F\left(X_{t_{1}}, \ldots, X_{t_{n}}\right)=F\left(X_{t_{1}+\beta}, \ldots, X_{t_{n}+\beta}\right)=F\left(X_{t_{1}}, \ldots, X_{t_{n}}\right)
$$

Logo $U(\beta)=I$ num conjunto denso em $L^{1}(Q, \Sigma, \mu)$. Assim, por continuidade da função $U(\beta)$, concluímos que $U(\beta)=I$ em $L^{1}(Q, \Sigma, \mu)$. Como $U(\beta)$ agindo em $L^{\infty}(Q, \Sigma, \mu)$ é a 
restrição de $U(\beta)$ agindo em $L^{1}(Q, \Sigma, \mu)$ concluímos que $U(\beta)=I$. Desta forma o espaço de trajetória generalizado é periódico de período $\beta$.

Denotando o sistema KMS estocasticamente positivo (de temperatura inversa $\beta$ ) denotado por $\left(\mathcal{B}, \mathcal{U}, \alpha_{t}, \omega\right), K$ o espectro de $\mathcal{U}$ e $Q=K^{[-\beta / 2, \beta / 2]}$, podemos enunciar o Teorema de KleinLandau por:

TeOrema DE Klein-Landau 4.3.1.3: $\operatorname{Seja}\left(\mathcal{B}, \mathcal{U}, \alpha_{t}, \omega\right)$ um sistema KMS estocasticamente positivo à temperatura inversa $\beta$. Então existe uma medida de probabilidade de Baire $\mu$ em $Q$, uma sub $\sigma$-álgebra $\Sigma_{0} \subset \Sigma$ (em que $\Sigma$ é a $\sigma$-álgebra de Borel de $Q$ ) um grupo $U(t)$ de automorfismos * em $L^{\infty}(Q, \Sigma, \mu)$ que preserva medidas e um automorfismo $R$ de $L^{\infty}(Q, \Sigma, \mu)$ que preserva medidas e tal que $\left(Q, \Sigma, \Sigma_{0}, U(t), R, \mu\right)$ é um espaço de trajetória generalizado OS-positivo e periódico de período $\beta$. Inversamente, dado um espaço de trajetória generalizado OS-positivo e periódico de período $\beta$, podemos construir um único, a não ser por isometrias, sistema KMS estocasticamente positivo à temperatura inversa $\beta$.

Agora vamos aplicar o conceito de espaço de trajetória generalizado mostrado anteriormente para explicar o efeito Unruh. Aqui deixamos claro que não se conseguiu cobrir todos os detalhes das demonstrações. O que acontece é que utilizaremos, para a explicação do efeito, as funções de Schwinger que são obtidas por extensões analíticas das funções de Wightman. Essas são, por sua vez, obtidas por operadores de campo, que não são limitados. Os trabalhos de [15] e [7] utilizam-se somente de operadores limitados. Assim nem tudo se encaixa de uma forma tão simples. Desta forma acreditamos que o resultado mostrado a seguir apresenta uma estratégia para uma possível demonstração alternativa deste efeito, que não foi obtida por problemas de natureza técnicas complicadas. Mostraremos, então, qual é esta estratégia.

Utilizando-se do espaço de trajetória generalizado podemos supor que $Q=\mathbb{S}^{\prime}$, em que $\mathbb{S}$ foi definido na seção 3.3. $\Sigma$ poderá ser considerada como a $\sigma$-álgebra de Borel e $\mu$ uma medida de probabilidade em $\mathbb{S}^{\prime}$.

Notamos que as funções $a \in \mathbb{S}$ podem ser pensadas como funções $\hat{a}: \mathbb{S}^{\prime} \rightarrow \mathbb{C}$ da seguinte forma: $\hat{a}(\varphi)=\varphi(a)$. Assim podemos naturalmente considerar $\Sigma_{0}$ como a $\sigma$-álgebra gerada por estas funções em $\mathbb{S}^{\prime}$, ou seja, a menor $\sigma$-álgebra tal que as funções $\hat{a}$ são mensuráveis. Agora gostaríamos de supor que exista um grupo de automorfismos $U(\theta)$ de operadores atuando nas funções $L^{\infty}(Q, \Sigma, \mu)$ tais que valha a seguinte igualdade abaixo:

$$
\phi_{2 \pi}\left(a_{1}, \theta_{1}, \ldots, a_{n}, \theta_{n}\right)=\int\left(U\left(\theta_{1}\right) \varphi\left(a_{1}\right)\right) \ldots\left(U\left(\theta_{n}\right) \varphi\left(a_{n}\right)\right) d \mu(\varphi) .
$$

Achamos que esta suposição não é totalmente artificial ou absurda, pois como se sabe, existem modelos em teorias quânticas de campos cujas funções de Schwinger são tais que existe uma medida de probabilidade, $\mu$, em $\mathcal{S}^{\prime}\left(\mathbb{R}^{2}\right)$ tal que para $f_{1}, \ldots, f_{n} \in \mathcal{S}_{\mathbb{R}}\left(\mathbb{R}^{2 n}\right)$ temos que 


$$
S\left(f_{1} \ldots f_{n}\right)=\int \varphi\left(f_{1}\right) \ldots \varphi\left(f_{n}\right) d \mu(\varphi) .
$$

Desta forma, vemos que o que estamos supondo é análogo, porém não se "integrou" $f$ em todas as variáveis, ou seja, $\theta$, continua sendo uma variável. Notemos, no entanto, que para fazer esta suposição usamos a comutatividade dos termos $(a, \theta)$. Logo estamos nos restringindo ao caso bosônico.

Podemos ver agora que a suposição acima é coerente com o fato de o espaço de trajetória ser periódico pois:

$$
\begin{gathered}
\left.\int U(2 \pi)\left[\left(U\left(\theta_{1}\right) \varphi\left(a_{1}\right)\right) \ldots\left(U\left(\theta_{n}\right) \varphi\left(a_{n}\right)\right)\right] d \mu(\varphi)=\int\left(U\left(2 \pi+\theta_{1}\right) \varphi\left(a_{1}\right)\right) \ldots\left(U\left(2 \pi+\theta_{n}\right) \varphi\left(a_{n}\right)\right)\right] d \mu(\varphi)= \\
\phi_{2 \pi}\left(a_{1}, 2 \pi+\theta_{1}, \ldots, a_{n}, 2 \pi+\theta_{n}\right)=\phi_{2 \pi}\left(a_{1}, \theta_{1}, \ldots, a_{n}, \theta_{n}\right)=\int\left(U\left(\theta_{1}\right) \varphi\left(a_{1}\right)\right) \ldots\left(U\left(\theta_{n}\right) \varphi\left(a_{n}\right)\right) d \mu(\varphi) .
\end{gathered}
$$

Logo $U(2 \pi)=i d$, em que $i d$ é a identidade e o espaço de trajetória é periódico de período $2 \pi$.

Da mesma forma, poderíamos supor a existência de um automorfismo $R$ nas funções $L^{\infty}$ tal que as funções $\hat{a} \in \mathbb{S}$ são invariantes por $R$, ou seja, $R \hat{a}=\hat{a}$. Este automorfismo seria tal que $U(-\theta) R=R U(\theta)$ e tal que

$$
\int R U(\theta) \varphi(a) d \mu=\phi_{2 \pi}(a,-\theta)
$$

Estas propriedades parecem coerentes pois

$$
\int U(-\theta) R \varphi(a) d \mu=\int U(-\theta) \varphi(a) d \mu=\phi_{2 \pi}(a,-\theta)=\int R U(\theta) \varphi(a) d \mu .
$$

Também vemos que é coerente pensar em $U(\theta)$ e $R$ como automorfismos, pois:

$$
\begin{gathered}
\left.\int U(\theta)\left[\left(U\left(\theta_{1}\right) \varphi\left(a_{1}\right)\right) \ldots\left(U\left(\theta_{n}\right) \varphi\left(a_{n}\right)\right)\right] d \mu(\varphi)=\int\left(U\left(\theta+\theta_{1}\right) \varphi\left(a_{1}\right)\right) \ldots\left(U\left(\theta+\theta_{n}\right) \varphi\left(a_{n}\right)\right)\right] d \mu(\varphi)= \\
\phi_{2 \pi}\left(a_{1}, \theta+\theta_{1}, \ldots, a_{n}, \theta+\theta_{n}\right)=\phi_{2 \pi}\left(a_{1}, \theta_{1}, \ldots, a_{n}, \theta_{n}\right)=\int\left(U\left(\theta_{1}\right) \varphi\left(a_{1}\right)\right) \ldots\left(U\left(\theta_{n}\right) \varphi\left(a_{n}\right)\right) d \mu(\varphi)
\end{gathered}
$$

$\mathrm{e}$ 


$$
\begin{aligned}
& \left.\int R\left[\left(U\left(\theta_{1}\right) \varphi\left(a_{1}\right)\right) \ldots\left(U\left(\theta_{n}\right) \varphi\left(a_{n}\right)\right)\right] d \mu(\varphi)=\int\left(U\left(-\theta_{1}\right) \varphi\left(a_{1}\right)\right) \ldots\left(U\left(-\theta_{n}\right) \varphi\left(a_{n}\right)\right)\right] d \mu(\varphi)= \\
& \phi_{2 \pi}\left(a_{1},-\theta_{1}, \ldots, a_{n},-\theta_{n}\right)=\phi_{2 \pi}\left(a_{1}, \theta_{1}, \ldots, a_{n}, \theta_{n}\right)=\int\left(U\left(\theta_{1}\right) \varphi\left(a_{1}\right)\right) \ldots\left(U\left(\theta_{n}\right) \varphi\left(a_{n}\right)\right) d \mu(\varphi) .
\end{aligned}
$$

Logo é plausível também pensar que $\|U(\theta) F\|_{1}=\|F\|_{1}$ e $\|R F\|_{1}=\|F\|_{1}$. Isto é coerente com os nossos resultados sobre estes operadores serem isometrias em $L^{p}$.

Também podemos ver que no limite em que $\theta$ vai a zero:

$$
\begin{gathered}
\int\left\{U(\theta)\left[\left(U\left(\theta_{1}\right) \varphi\left(a_{1}\right)\right) \ldots\left(U\left(\theta_{n}\right) \varphi\left(a_{n}\right)\right)\right]-\left[\left(U\left(\theta_{1}\right) \varphi\left(a_{1}\right)\right) \ldots\left(U\left(\theta_{n}\right) \varphi\left(a_{n}\right)\right)\right]\right\} d \mu(\varphi)= \\
\phi_{2 \pi}\left(a_{1}, \theta+\theta_{1}, \ldots, a_{n}, \theta+\theta_{n}\right)-\phi_{2 \pi}\left(a_{1}, \theta_{1}, \ldots, a_{n}, \theta_{n}\right) \rightarrow 0 .
\end{gathered}
$$

Assim nos parece que $U(\theta)$ é fortemente contínuo.

Por fim, a última característica que gostaríamos de mostrar é que estas definições indicam que valha a OS-positividade, pois se $\theta_{1}, . ., \theta_{n} \in[0, \beta / 2]$ então

$$
\begin{gathered}
\int R\left[\left(U\left(\theta_{1}\right) \varphi\left(a_{1}^{*}\right)\right) \ldots\left(U\left(\theta_{n}\right) \varphi\left(a_{n}^{*}\right)\right)\right]\left(U\left(\theta_{1}\right) \varphi\left(a_{1}\right)\right) \ldots\left(U\left(\theta_{n}\right) \varphi\left(a_{n}\right)\right) d \mu= \\
=\phi_{2 \pi}\left(a_{1}^{*},-\theta_{1}, \ldots, a_{n}^{*},-\theta_{n}, a_{1}, \theta_{1}, \ldots, a_{n}, \theta_{n}\right)=\phi_{2 \pi}\left(a_{1}, \theta_{1}, \ldots, a_{n}, \theta_{n}, a_{1}^{*}, 2 \pi-\theta_{1}, \ldots, a_{n}^{*}, 2 \pi-\theta_{n}\right) \geq 0 .
\end{gathered}
$$

Desta forma, permitimo-nos pensar que existe uma possibilidade de que tal operadores e um tal espaço de probabilidade sejam construídos e satisfaçam 4.3.

De forma resumida, nossas conjecturas foram a existência de uma medida em $\mathbb{S}^{\prime}$, com sua álgebra de Borel $\Sigma$, e de automorfismos $U(\theta)$ e $R$ em que satisfaçam as equações 4.3 e 4.4. Usamos em seguida as propriedades (P1) até (P4) da seção 3.3 para argumentar que estas propriedades são análogas as propriedades requeridas para definir um espaço de trajetória generalizado. Assim achamos plausível que com isto seja possível aplicar o Teorema 4.3.1.3.

A seguir mostraremos como estas propriedades podem ser usadas para a construção de um sistema KMS.

\subsubsection{O Teorema de reconstrução}

Como vimos acima, parece-nos plausível aplicar o Teorema 4.3.1.3. para o espaço de trajetória $\mathbb{S}^{\prime}$ definido acima. Uma vez que podemos aplicá-lo, ainda que de forma não completamente 
satisfatória, vamos esboçar, sem entrar nas minúcias, como se obter o espaço de Hilbert físico e o sistema KMS estocasticamente positivo a partir de $\left(Q, \Sigma, \Sigma_{0}, U(t), R, \mu\right)$, em que este é um espaço de trajetória generalizado OS-positivo e periódico de período $\beta$. Vamos seguir aqui $[7,15]$.

Consideremos o espaço de Hilbert $L^{2}\left(Q, \Sigma_{[0, \beta / 2]}, \mu\right)$, em que $\Sigma_{[0, \beta / 2]}=\bigvee_{t \in[0, \beta / 2]} U(t) \Sigma_{0}$ e o operador $E_{[0, \beta / 2]} R E_{[0, \beta / 2]}$ que é positivo (OS-positividade) e age neste espaço. Agora podemos definir um produto interno da seguinte forma para elementos em $L^{2}\left(Q, \Sigma_{[0, \beta / 2]}, \mu\right)$ :

$$
(F, G):=\int_{Q} R(\bar{F}) G d \mu=\int_{Q}\left(E_{[0, \beta / 2]} R E_{[0, \beta / 2]} \bar{F}\right) G d \mu .
$$

Podemos ver agora que este produto interno satisfaz $(F, F) \geq 0$ pois $\left\langle(R F)^{*} F\right\rangle \geq 0$ devido a positividade OS (chamada de positividade por reflexão em algumas referências). Também vemos que $(F, F) \leq\|F\|_{L^{2}}$, conforme se pode ver abaixo:

$$
(F, F)=\langle R(\bar{F}) F\rangle=\langle\bar{F} R(F)\rangle \leq\langle|\bar{F} R(F)|\rangle \leq \sqrt{\int\left|F^{*}\right|^{2} d \mu} \sqrt{\int|R F|^{2} d \mu} \leq\|F\|_{L^{2}}
$$

Na segunda igualdade usou-se o fato de $\mathrm{R}$ ser uma isometria. O espaço de Hilbert físico será dado por $\mathcal{H}=$ completamento de $L^{2}\left(Q, \Sigma_{[0, \beta / 2]}, \mu\right) / \mathcal{N}$, em que $\mathcal{N}$ é o subespaço dos vetores $G$ tais que $(G, G)=0$. Se denotarmos por $\mathcal{V}$ a projeção canônica de $L^{2}\left(Q, \Sigma_{[0, \beta / 2]}, \mu\right)$ em $L^{2}\left(Q, \Sigma_{[0, \beta / 2]}, \mu\right) / \mathcal{N}$, podemos estendê-la unicamente a uma contração. O espaço de Hilbert $L^{2}\left(Q, \Sigma_{[0, \beta / 2]}, \mu\right) / \mathcal{N}$ será o espaço de Hilbert físico e o vetor $\Omega:=\mathcal{V}(1)$ será o vácuo. O passo seguinte será o de achar $\mathcal{U}$. Estes operadores correspondem, de certa maneira, aos operadores de multiplicação por $L^{\infty}\left(Q, \Sigma_{0}, \mu\right)$. A seguir vamos enunciar o Teorema de Klein a este respeito:

TeOrema 4.3.2.1 [15]: Para $F \in L^{2}\left(Q, \Sigma_{[0, \beta / 2]}, \mu\right)$ e $f \in L^{\infty}\left(Q, \Sigma_{0}, \mu\right)$ podemos definir o operador $\tilde{f}$ em $\mathcal{H}$ por $\tilde{f} \mathcal{V}(F)=\mathcal{V}(f F)$. Este operador é tal que $\|\tilde{f}\|=\|f\|_{\infty}$. Além disto $\overline{\mathcal{U}}=\left\{\tilde{f} ; f \in L^{\infty}\left(Q, \Sigma_{0}, \mu\right)\right\}$ é uma álgebra de von Neumann de operadores em $\mathcal{H}$ tendo $\Omega$ com vetor separante.

O próximo passo é achar $\mathcal{B}$ e o operador de evolução temporal. Basicamente o que se mostrará é que $U(t)$ pode agir em $\mathcal{H}$ de uma forma "canônica" e que ele é o operador de evolução temporal imaginária. Para ver como isto funciona vamos primeiramente definir um semigrupo local simétrico. Este semigrupo corresponderá de certa forma a $U(t)$ e de lá obteremos suas propriedades.

DeFIniçÃo 4.3.2.2 [15]: Seja $\mathcal{H}$ um espaço de Hilbert e $T$ um número real $>0$. Um semigrupo simétrico local $\left(P(t), \mathcal{D}_{t}, T\right)$ é uma família $\left\{P(t), \mathcal{D}_{t}\right\}_{t \in[0, T]}$ de operadores lineares $P(t)$ e subespaços vetoriais $\mathcal{D}_{t}$ de $\mathcal{H}$ tais que

(i) $\mathcal{D}_{0}=\mathcal{H}, \mathcal{D}_{t} \supset \mathcal{D}_{s}$ se $0 \leq t \leq s \leq T$ e $\mathcal{D}=\cup_{0<t \leq T} \mathcal{D}_{t}$ é denso em $\mathcal{H}$. 
(ii) $P(t): \mathcal{D}_{t} \rightarrow \mathcal{H}$ é um operador linear e simétrico (ou seja, $(u, P(t) v)=(P(t) u, v)$ para todo $u, v \in \mathcal{D}_{t}$ ) em que $P(0)=I$ e $P(s) \mathcal{D}_{t} \subset \mathcal{D}_{t-s}$ para $0 \leq s \leq t \leq T$ e $P(t) P(s)=P(t+s)$ em $\mathcal{D}_{t+s}$ para $t, s, t+s \in[0, T]$.

(iii) $t \rightarrow P(t)$ é fracamente contínuo, isto é, para $u \in \mathcal{D}_{s}$ e $0 \leq t \leq s$ o mapa $t \rightarrow(u, P(t) u)$ é contínuo.

O fato crucial a respeito destes semigrupos locais é que o operador $P(t)$ pode ser escrito como $e^{-t L}$. O operador $L$ é auto-adjunto e será o Lioviliano do sistema KMS. Para ser mais preciso vamos estabelecer a relação entre $P(t)$ e $e^{-t L}$ no Teorema abaixo:

TeOrema 4.3.2.3 [15]: Seja $\left(P(t), \mathcal{D}_{t}, T\right)$ um semigrupo local simétrico em $\mathcal{H}$. Então existe um único operador auto-adjunto $L$ em $\mathcal{H}$ tal que

(i) $\mathcal{D}_{t} \subset \mathcal{D}\left(e^{-t L}\right),\left.e^{-t L}\right|_{\mathcal{D}_{t}}=P(t)$ para $0 \leq t \leq T$, ou seja, e $e^{-t L}$ é uma extensão de $P(t)$.

(ii) $\mathcal{D}_{\left.] 0, T^{\prime}\right]}:=\cup_{0<t \leq T^{\prime}} \cup_{0<s<t} P(s) \mathcal{D}_{t}$ é um núcleo para $L$ para $0<T^{\prime} \leq T$.

Agora com a descrição dos operadores acima podemos estabelecer a relação de $U(t)$ e $P(t)$. O objetivo é mostrar que $U(t)$ "age" em $\mathcal{H}$ como um semigrupo local simétrico. Vamos inicialmente definir para $0 \leq t \leq \beta / 2$ o subespaço $\mathcal{M}_{t}:=L^{2}\left(Q, \Sigma_{[0, \beta / 2-t]}, \mu\right)$ de $L^{2}\left(Q, \Sigma_{[0, \beta / 2]}, \mu\right)$. O subespaço $\mathcal{D}_{t}$ acima será dado por $\mathcal{V}\left(\mathcal{M}_{t}\right)$ e o operador $P(s)$ agirá em $P(s): \mathcal{D}_{t} \rightarrow \mathcal{D}_{t-s}$ e será dado por $P(s) \mathcal{V}(F)=\mathcal{V}(U(s) F)$. Assim vemos que o operador $P(s)$ é, de certa forma, o operador $U(s)$ "agindo" em $\mathcal{H}$. Para tornar toda idéia mais precisa vamos enunciar isto num Teorema de [15] tal como está enunciado em [7]:

TeOREma 4.3.2.4 [7, 15]: Seja $\left(Q, \Sigma, \Sigma_{0}, U(t), R, \mu\right)$ um espaço de trajetórias OS-positivo e de periodo $\beta$. Definindo $\mathcal{M}_{t}:=L^{2}\left(Q, \Sigma_{[0, \beta / 2]}, \mu\right)$ para $0 \leq t \leq \beta / 2$. Então

(i) $U(s): \mathcal{M}_{t} \cap \mathcal{N} \rightarrow \mathcal{M}_{t-s} \cap \mathcal{N}$ para $0 \leq s \leq t \leq \beta / 2$. Se $\mathcal{D}_{t}:=\mathcal{V}\left(\mathcal{M}_{t}\right)$, então o operador linear $P(s)$ dado por:

$$
P(s): \begin{aligned}
\mathcal{D}_{t} & \rightarrow \mathcal{D}_{t-s} \\
\mathcal{V}(F) & \rightarrow \mathcal{V}(U(s) F)
\end{aligned}
$$

é bem definido. (Veja que a razão disto é que se $\mathcal{V}(F)=0$ então $(F, F)=0$, logo $(U(s) F, U(s) F)=$ 0 e assim $P(s) \mathcal{V}(F)=0$. Logo como $P(s)$ é linear, ele está bem definido.)

(ii) $\left(P(t), \mathcal{D}_{t}, \beta / 2\right)$ é um semigrupo local simétrico.

Conclui-se assim que existe um único operador auto-adjunto $L$ tal que $\left.P(t)\right|_{\mathcal{D}_{t}}=e^{-t L}$. Além disto $L \Omega=0$.

Com estes resultados podemos definir $\mathcal{B}$ como a álgebra de von Neumann gerada por $\left\{e^{i t L} A e^{-i t L} ; A \in \mathcal{U}, t \in \mathbb{R}\right\}$. O operador de evolução temporal será definido por $\tau_{t}(A)=$ $e^{i t L} A e^{-i t L}$ para $A$ em $\mathcal{B}$. Com estas definições enfim consegue-se construir o sistema KMS estocasticamente positivo tal qual vamos enunciar a seguir: 
TeOrema 4.3.2.5 [7, 15]: Seja $\omega_{\Omega}$ um estado em $\mathcal{B}$ definido por $\omega_{\Omega}(B)=(\Omega, B \Omega)$. Então $\left(\mathcal{B}, \mathcal{U}, \tau, \omega_{\Omega}\right)$ é um sistema $K M S$ estocasticamente positivo com periodo $\beta$.

Um fato interessante provado por [15] se refere ao Teorema de Tomita-Takesaki. Com os resultados deste artigo pode-se afirmar que o operador modular é dado por $\Delta=e^{\beta L}$ e o operador de conjugação é dado por $J \mathcal{V}(F)=\mathcal{V}\left(R_{\beta / 4} \bar{F}\right)$, em que $R_{\beta / 4}:=U(\beta / 4) R U(-\beta / 4)=$ $R U(-\beta / 2)=U(\beta / 2) R$.

Podemos agora relacionar o Teorema de reconstrução com a discussão anterior do efeito Unruh. Como vimos lá, o operador $U(t)$ deve ser entendido como $U(\theta)$ tal como definido naquela seção, que por sua vez corresponde a uma rotação de um ângulo $\theta$ ao longo dos eixos $x$ e it. Como vimos nesta seção o operador $U(\theta)$ tem uma associação "canônica" com o operador $P(\theta)$ que age em $\mathcal{H}=$ completamento de $L^{2}\left(Q, \Sigma_{[0, \beta / 2]}, \mu\right) / \mathcal{N}$. No caso do efeito Unruh teríamos $\beta=2 \pi$, já que o espaço de trajetória generalizado é $2 \pi$-periódico naquele caso.

Vimos assim que o operador $P(\theta)=e^{L \theta}$, em que $L$ é um operador auto-adjunto. Sabemos pelas discussões na seções anteriores que a rotação por um ângulo $\theta$ ao longo dos eixos $x$ e it corresponde a um boost de Lorentz "complexo". Assim se o boost de Lorentz na direção $x$ é dado por $t \rightarrow t \cosh \tau+x \sinh \tau$ e $x \rightarrow x \cosh \tau+t \sinh \tau$, então sua representação no espaço de Hilbert deve ser dado por um operador unitário $e^{i \tau F}$. Se $\tau$ for complexo, ou seja, $\tau \rightarrow-i \theta$ (o sinal - é apenas uma convenção), obtemos $e^{F \theta}$. Como esta complexificação corresponde justamente a uma rotação nos eixos $x$ e it, concluímos que $F=L$. Logo podemos interpretar o operador $L$ como o gerador dos boosts de Lorentz, no caso do efeito Unruh. A evolução temporal é, então, $e^{i L t}$, ou seja, corresponde aos boosts. Estes, como sabemos, correspondem aos observadores uniformemente acelerados.

Concluímos assim que associado à evolução temporal dada pelos boosts existe um estado KMS. Do ponto de vista físico podemos entender da seguinte forma. Sabemos que dadas as funções de Schwinger, podemos reconstruir o espaço de Hilbert físico. Consideramos, para isto, o espaço de Hilbert sendo dado por seqüências:

$$
\underline{f}=\left(f_{0}, f_{1}\left(y_{1}\right), \ldots, f_{n}\left(y_{1}, \ldots, y_{n}\right), 0,0, \ldots\right) .
$$

Ou seja, seqüências com apenas finitos termos diferentes de 0 e tais que $f_{j} \in \mathcal{S}_{+}\left(\mathbb{R}^{4 j}\right)$, em que este é o espaço de Schwartz das funções com suporte em tempos positivos. Com seqüências desta forma, podemos definir um produto interno neste espaço dado por:

$$
<\underline{f}, \underline{g}>^{E}=\sum_{n, m} S_{n+m}\left(\theta f_{n}^{*} \times f_{m}\right)
$$

em que $\theta f_{n}\left(t_{1}, \vec{x}_{1}, \ldots, t_{n}, \vec{x}_{n}\right)=f_{n}\left(-t_{1}, \vec{x}_{1}, \ldots,-t_{n}, \vec{x}_{n}\right)$ e $f_{n}^{*}\left(t_{1}, \vec{x}_{1}, \ldots, t_{n}, \vec{x}_{n}\right)$ é, por definição, igual a $\bar{f}_{n}\left(t_{n}, \vec{x}_{n}, \ldots, t_{1}, \vec{x}_{1}\right)$. O espaço de Hilbert físico será o completamento canônico deste espaço. Notemos então que esta expressão é análoga à 4.5, porém a reflexão é no tempo imaginário de observadores inerciais. O vácuo neste caso será o vácuo da teoria de campos a temperatura 0, para 
observadores em referências inerciais. Assim vemos que o vácuo construído por observadores inerciais é de temperatura 0, enquanto que o vácuo construído por observadores uniformemente acelerados tem temperatura finita. Interpretamos assim que o vácuo de Minkowski visto por observadores uniformemente acelerados é um estado de temperatura finita, ou seja, obtemos o efeito Unruh. 


\section{Capítulo 5}

\section{Conclusão}

No trabalho apresentado, buscamos fazer um panorama acerca do efeito Unruh mostrando diferentes formas de entendê-lo. Basicamente foram estudadas três explicações do efeito Unruh para partículas bosônicas. Na primeira, o efeito foi estudado sob a perspectiva da teoria quântica de campos tal qual ela é normalmente apresentada nos cursos de física. Esta visão se assemelha bastante com a forma original que W.G. Unruh [32] descobriu o efeito que leva seu nome. Na segunda forma, procurou-se entender o efeito sobre um ponto de vista algébrico, baseando-se basicamente no trabalho de G. Sewell [27]. Por fim propôs-se uma nova estratégia para sua compreensão, baseando-se em métodos Euclidianos de Teoria Quântica de Campos.

Em cada uma destas etapas foram estudadas certas características inerentes à explicação considerada. Em cada uma destas explicações obtiveram-se pequenos resultados que, apesar de em geral conhecidos, nem sempre são facilmente encontrados.

Assim no primeiro ponto de vista estudado, buscou-se obter um panorama geral da quantização em espaços curvos através do modelo de campo livre bosônico complexo. Também comparamos o formalismo adotado com a forma de quantizar campos lineares estudada por Dimock [3]. Como resultado deste estudo, procurou-se entender, ao menos de uma forma simplificada, conceitos como o de hiperbolicidade global, isometrias em espaços-tempos etc, assim como suas aplicações e importância para a formulação da teoria em espaços-tempos curvos.

Neste primeiro ponto de vista estudado, buscamos fazer contas explícitas dos resultados necessários para a explicação do efeito. Assim mostramos explicitamente porque os boosts ou as translações temporais correspondem a isometrias, assim como foram calculados explicitamente os coeficientes de Bogoliubov, baseando-se nas notas das aulas do professor V. Mukhanov [20]. Acreditamos que esta seja uma forma importante de entender o problema, mesmo que para fins de um trabalho de física-matemática, devido a semelhança e suas relações com o que é apreendido nos cursos de teoria de campos.

O segundo ponto de vista estudado é considerado uma forma "rigorosa" de entender o efeito. Ela de fato é, no sentido matemático, mais precisa que a anterior. Esta explicação requer um conhecimento mais profundo de algumas áreas na matemática. Como conseqüência, foi realizado um estudo sobre fundamentos das álgebras de operadores. O resumo dos fatos 
estudados mais importantes ao trabalho foi adicionado ao texto. Também adicionamos fatos básicos sobre a chamada teoria axiomática de campos e uma descrição razoavelmente autosuficiente sobre estados de equilíbrio. Nesta parte, aprendemos como fazer a descrição destes estados e obtivemos demonstrações de fatos conhecidos, mas que não são encontrados facilmente nem de forma tão explícita na literatura como a mostrada.

Acreditamos que em relação à segunda parte houve um bom aproveitamento num nível iniciante. As provas completadas ou elaboradas no estudo dos estados KMS foram de um nível matemático razoavelmente elementar, no entanto elas são extremamente importante para qualquer aplicação deste conceito (de estado KMS). Cremos também que a compreensão do trabalho de G. Sewell e, portanto, do efeito foi bastante satisfatória. Isto abre caminhos para estudos das generalizações deste efeito feito também no trabalho de Sewell e mais recentemente por Robert Wald e Bernard Kay [19]. Estas são generalizações mais complicadas que envolvem o conceito de horizonte de Killing entre outras coisas. No entanto, pelo menos em relação ao trabalho de Wald e Kay, trata-se de um trabalho recente, mostrando o contínuo interesse que este efeito ainda desperta.

A terceira parte apresentou uma nova estratégia para demonstração do efeito Unruh. Acreditamos que ela forneceu um interessante ponto de vista acerca deste fenômeno físico, partindo de técnicas Euclidianas bastante úteis em teoria de campos.

O ponto de vista Euclidiano é um caminho para uma compreensão de diversas teorias físicas e suas relações, tais quais as existentes entre mecânica estatística clássica e teorias quânticas de campos. Ele também fornece um rico material matemático que envolve teoria de probabilidades, processos estocásticos, entre outros, que têm uma importância sozinha bastante grande.

Nesta terceira parte, procuramos estudar os fundamentos destas técnicas, além de outras já consagradas na física-matemática, tais como princípios de análise funcional além de álgebra de operadores. Cremos que foi possível ter uma boa visão geral ao menos dos fundamentos dessas áreas de pesquisa. Isto permite uma maior perspectiva de aplicações no futuro. Deste estudo se destacam as relações entre análise funcional e probabilidade. As relações mostradas no trabalho são conhecidas e utilizadas por diversos outros. No entanto, não foi encontrada uma referência que mostre todos os resultados apresentados. Algumas provas, portanto, são originais, embora seus resultados já sejam conhecidos. Muitas provas foram refeitas também, acrescentando mais detalhes.

Além dos fundamentos destas técnicas, foram estudados dois interessante artigos: do KleinLandau [15], com relativa profundidade e do Gérard-Jäkel [7], de forma menos detalhada. Estes artigos, além de fornecerem uma valiosa ilustração das técnicas abordadas, abrem portas para pesquisas bastante atuais. De acordo com Gérard-Jäkel [7], a parte construtivista para campos a temperaturas finitas, que usa muito das técnicas apresentadas, mal começou, sendo algo ainda a ser explorado.

Infelizmente, embora as técnicas apresentadas sejam bastante poderosas elas são também bastante complicadas, impedindo que as demonstrações de efeitos de relevância física, como o 
apresentado no trabalho, sejam levadas a cabo num nível ainda iniciante. No entanto, é possível ter algumas idéias de como prosseguir para uma demonstração completa do efeito, usando nossa estratégia.

Inicialmente, um conhecimento mais profundo das técnicas citadas acima poderia facilitar a aplicação delas, além de permitir uma visão mais madura dos artigos estudados.

Mesmo com esta compreensão, devemos lembrar que os trabalhos [15, 7] já partem de estados KMS para campos livres, estados KMS quase-livres no caso de [7], e usam assim operadores limitados para descrever o estado de equilíbrio. Assim, seria interessante saber como os operadores não-limitados dos campos de Wightman se relacionam com os operadores limitados dos estados de equilíbrio. Ou, então, saber como fazer a "Euclidianização" dos valores esperados da álgebra de Weyl dos operadores de campo, o que faria com que sempre utilizássemos operadores limitados.

De qualquer maneira, o entendimento de qualquer um desses fatos levaria muito mais tempo e extrapolaria as intenções desta tese.

Em suma, acreditamos que o trabalho, embora incompleto no sentido de dar demonstrações até o fim, abre perspectivas bastante amplas. Ele fornece três perspectivas de um mesmo efeito, abordando em cada uma delas aspectos fundamentais da teoria. Além disto acreditamos que fornece uma estratégia estimulante para a compreensão do efeito físico estudado. 


\section{Referências Bibliográficas}

[1] L. Breiman. Probability. SIAM: Society for Industrial and Applied Mathematics, 1992. Reprint edition.

[2] K. L. Chung. A course in Probability Theory. Academic Press, 2001. terceira edição.

[3] J. Dimock. Algebras of local observables on a manifold. Communications in Mathematical Physics, 77:219, 1980.

[4] J. L. Doob. Stochastic Processes. Wiley, 1953. Wiley Publications in Statistics.

[5] J. Glimm e A. Jaffe. Quantum Physics: A Functional Integral Point of View. Springer, 1987. segunda edição.

[6] M. Reed e B. Simon. Functional Analysis. Academic Press, 1980. Edição Revisada.

[7] C. Gérard e C. D. Jäkel. Thermal quantum fields with spatially cut-off interactions in $1+1$ space-time dimensions. http://www.math.u-psud.fr/ gerard/, 2003.

[8] O. Bratteli e D.W. Robison. Operator Algebras and Quantum Statistical Mechanics I and II. Springer-Verlag, 1981.

[9] J.J Bisognano e E. H. Wichmann. On the duality condition for a hermitian scalar field. Journal of Mathematical Physics, 16:985-1007, 1975.

[10] J.J Bisognano e E. H. Wichmann. On the duality condition for quantum fields. Journal of Mathematical Physics, 17:303-321, 1976.

[11] L. Landau e E. Lifschitz. Teoria do Campo. Editora Mir, 1980.

[12] L. Birke e J. Fröhlich. Kms, etc. Reviews in Mathematical Physics, 14:829-871, 2002.

[13] R. V. Kadison e J. R. Ringrose. Fundamentals of the Theory of Operator Algebras Volume II: Advanced Theory. American Mathematical Society, 1983. segunda edição.

[14] R. V. Kadison e J. R. Ringrose. Fundamentals of the Theory of Operator Algebras Volume:1 Elementary Theory. American Mathematical Society, 1983. 
[15] A. Klein e L. J. Landau. Stochastic processes associated with kms states. Journal of Functional Analysis, 42:368-428, 1981.

[16] N.D. Birrel e P.C.W. Davies. Quantum Fields in Curved Spaces. Cambridge University Press, 1982.

[17] K. Osterwalder e R. Schrader. Axioms for euclidean green's functions. Communications in Mathematical Physics, 31:83-112, 1973.

[18] A. Wightmann e R.F.Streater. PCT, Spin, Statistics and All That. Benjamin, 1964.

[19] B.S. Kay e R.M.Wald. Theorems on the uniqueness and thermal properties of stationary, nonsingular quasifree states on spacetimes with a bifurcate killing horizon. Physics Reports, 207:49, 1991.

[20] V. Mukhanov e S. Winitzki. Notas de aula de um curso de teoria de campos em espaços curvos. http://www.theorie.physik.uni-muenchen.de/ serge/T6/.

[21] P. J. Fernandez. Medida e Integração. IMPA, 1976. Projeto Euclides.

[22] R. Haag. Local Quantum Physics. Springer Verlag, 1996. segunda edição.

[23] S. Hawking. Particle creation by black holes. Communications in Mathematical Physics, 43:199, 1975.

[24] R. Jost. The general theory of quantized fields. American Mathematical Society, 1965. Lectures in applied mathematics.

[25] L. Parker. Quantized fields and particle creation in expanding universes. Physical Review, 183:1057, 1969.

[26] M. Winnink R. Haag, N.M. Hugenholtz. On the equilibrium states in quantum statistical mechanics. Communications in Mathematical Physics, 5:215, 1967.

[27] G. L. Sewell. Quantum fields on manifolds. Annals of Physics, 141:201-224, 1982.

[28] B. Simon. The P( $\phi$ )2 Euclidean (Quantum) Field Thery. Princeton University Press, 1974.

[29] F. Strocchi. Selected Topics on the General Properties of Quantum Field Theory. World Scientific, 1993.

[30] M. Takesaki. Tomita's Theory of Modular Hilbert Algebras and its Applications. SpringerVerlag, 1970. Lecture Notes in Mathematics 128.

[31] D. V. Tausk. Notas de Aula de Medida e Integração. http://www.ime.usp.br/ tausk/texts/NotasMedida.pdf. 
[32] W.G. Unruh. Notes on black hole evaporation. Physical Review D, 14:870, 1976.

[33] R. M. Wald. General Relativity. University of Chicago Press, 1983.

[34] R. M. Wald. Quantum Field Theory in Curved Spacetime and Black Hole Thermodynamics. University of Chicago Press, 1994. 